\title{
Reinforced Topological Nano-assemblies: 2D Hexagon-Fused Wheel to 3D Prismatic Metallo-lamellar Structure with Molecular Weight of 119K Daltons
}

Guotao Wang, ${ }^{\dagger, \#}$ Mingzhao Chen,,,,,$+ \#$ Jun Wang, ${ }^{\dagger}$ Zhiyuan Jiang, ${ }^{\dagger}$ Die Liu, ${ }^{\dagger}$ Dongyang Lou, ${ }^{\dagger}$ He Zhao, ${ }^{\dagger}$ Kaixiu Li,${ }^{\dagger}$ Suqing Li, ${ }^{\dagger}$ Tun Wu, ${ }^{\dagger}$ Zhilong Jiang,,${ }^{\dagger}$ Xiaoyi Sun ${ }^{\dagger}$ and Pingshan Wang $*, \dagger, \dagger$

†Department of Organic and Polymer Chemistry; Hunan Key Laboratory of Micro \& Nano Materials Interface Science, College of Chemistry and Chemical Engineering; Central South University, Changsha, Hunan-410083, China

*Institute of Environmental Research at Greater Bay Area; Key Laboratory for Water Quality and Conservation of the Pearl River Delta, Ministry of Education; Guangzhou Key Laboratory for Clean Energy and Materials, Guangzhou University, Guangzhou-510006, China

Table of Contents

1. General procedures 2

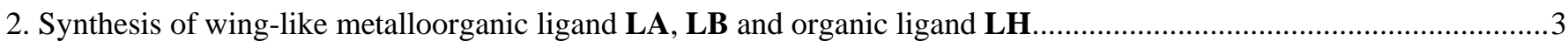

3. Self-Assembly of Hexagon-Fused Wheel W1 and Prismatic Lamellar Structure P1 .............................................5

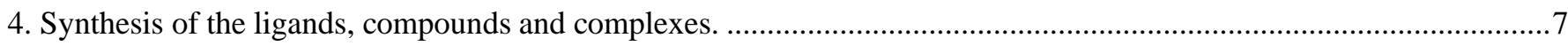

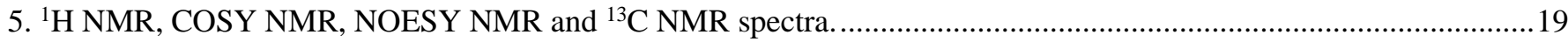

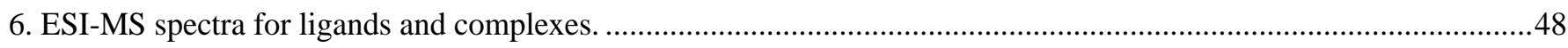

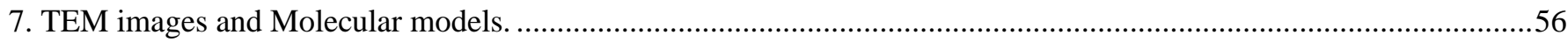

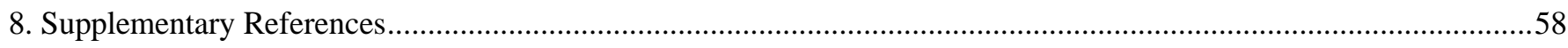




\section{General procedures}

All starting materials were purchased from Aldrich, Alfa Aesar and used without further purification. organic ligand LH, compound 6, compound S2 and compound S6 were synthesized according to the literature procedures. ${ }^{\text {S1-4 }}$ And 5bromo-2-iodo-1,3-dimethoxybenzene were synthesized according to the patent (WO 2008079836). Solvents used in the experimental processes were purified prior to use. 5-bromo-1,3-dimethoxy-2-nitrobenzene, (5-bromo-2,4dimethoxyphenyl)boronic acid, 1,3-dibromobenzene, $\mathrm{LiN}\left(\mathrm{SO}_{2} \mathrm{CF}_{3}\right)_{2}$, and $\mathrm{RuCl}_{3} \cdot 3 \mathrm{H}_{2} \mathrm{O}$ and other materials were directly purchased from J \& K Chemical Technology and used without further purification. Analytical thin layer chromatography (TLC) was performed on aluminum-backed sheets precoated with $\mathrm{Al}_{2} \mathrm{O}_{3}$ (150 F254 adsorbent, $0.25 \mathrm{~mm}$ thick; Merck, Germany). Column chromatography was conducted using neutral $\mathrm{Al}_{2} \mathrm{O}_{3}$ (200-300 mesh) or $\mathrm{SiO}_{2}$ from Sinopharm Chemical Reagent Co. The ${ }^{1} \mathrm{H}$ NMR spectra were recorded at $25^{\circ} \mathrm{C}$ on a Bruker spectrometer operating at either 500 or $400 \mathrm{MHz}$ for ${ }^{1} \mathrm{H}$ or ${ }^{13} \mathrm{C}$, respectively. Chemical shifts were reported in parts per million (ppm) referenced to the residual solvent peak for ${ }^{1} \mathrm{H}$ and ${ }^{13} \mathrm{C}$ NMR, respectively. Transmission electron microscopy measurements were performed on a JEM-2100F TEM operating at $200 \mathrm{kV}$, the sample was dissolved in MeCN at a concentration of $\sim 10^{-6} \mathrm{M}$. The solutions were drop cast onto a carbon-coated $\mathrm{Cu}$ grid (300-400 mesh) and extra solution was absorbed by filter paper to avoid aggregation, images were taken with a JEOL 2010 Transmission Electron Microscope. Scanning electron microscopy (SEM) was performed on a Helios Nanolab 600i. Electro-spray ionization (ESI) mass spectra were recorded with a Bruker Q-TOF Qualification Standard Kit. or Waters Synapt G2i Mass Spectrometer, using solutions of $0.1 \mathrm{mg}$ sample in $10 \mathrm{~mL}$ of $\mathrm{CHCl}_{3} / \mathrm{MeCN}(1: 3, \mathrm{v} / \mathrm{v})$ for ligands and different concentrations for complexes. The TWIM MS experiments were performed under the following conditions: ESI capillary voltage, $3.5 \mathrm{kV}$; sample cone voltage, $60 \mathrm{~V}$; source temperature $100^{\circ} \mathrm{C}$; desolvation temperature, $300^{\circ} \mathrm{C}$; cone gas flow, $6 \mathrm{~L} / \mathrm{h}$; desolvation gas flow, $700 \mathrm{~L} / \mathrm{h}(\mathrm{N} 2)$; source gas control, 0 $\mathrm{mL} / \mathrm{min}$; trap gas control, $1.6 \mathrm{~mL} / \mathrm{min}$; Helium cell gas control, $160 \mathrm{~mL} / \mathrm{min}$; ion mobility (IM) cell gas control, $50 \mathrm{~mL} / \mathrm{min}$; sample flow rate, $5 \mu \mathrm{L} / \mathrm{min}$; IM traveling wave height, $40 \mathrm{~V}$; and IM traveling wave velocity, $650 \mathrm{~m} / \mathrm{s}$. UV-vis absorption spectra were recorded with an Agilent 8453 UV-vis Spectrometer. The rheological property and self-healing of supramolecular polymer gels were tested by a rheometer (Anton Paar, 302, GmbH). The structural optimization and energy calculation were carried out in Materials Studio version 6.1, using the Geometry Optimization and Energy tasks in the Forcite module (Accelrys Software, Inc.). The initial structural model was built up with all counterions omitted for clarity. 


\section{Synthesis of wing-like metalloorganic ligand LA, LB and organic ligand LH}
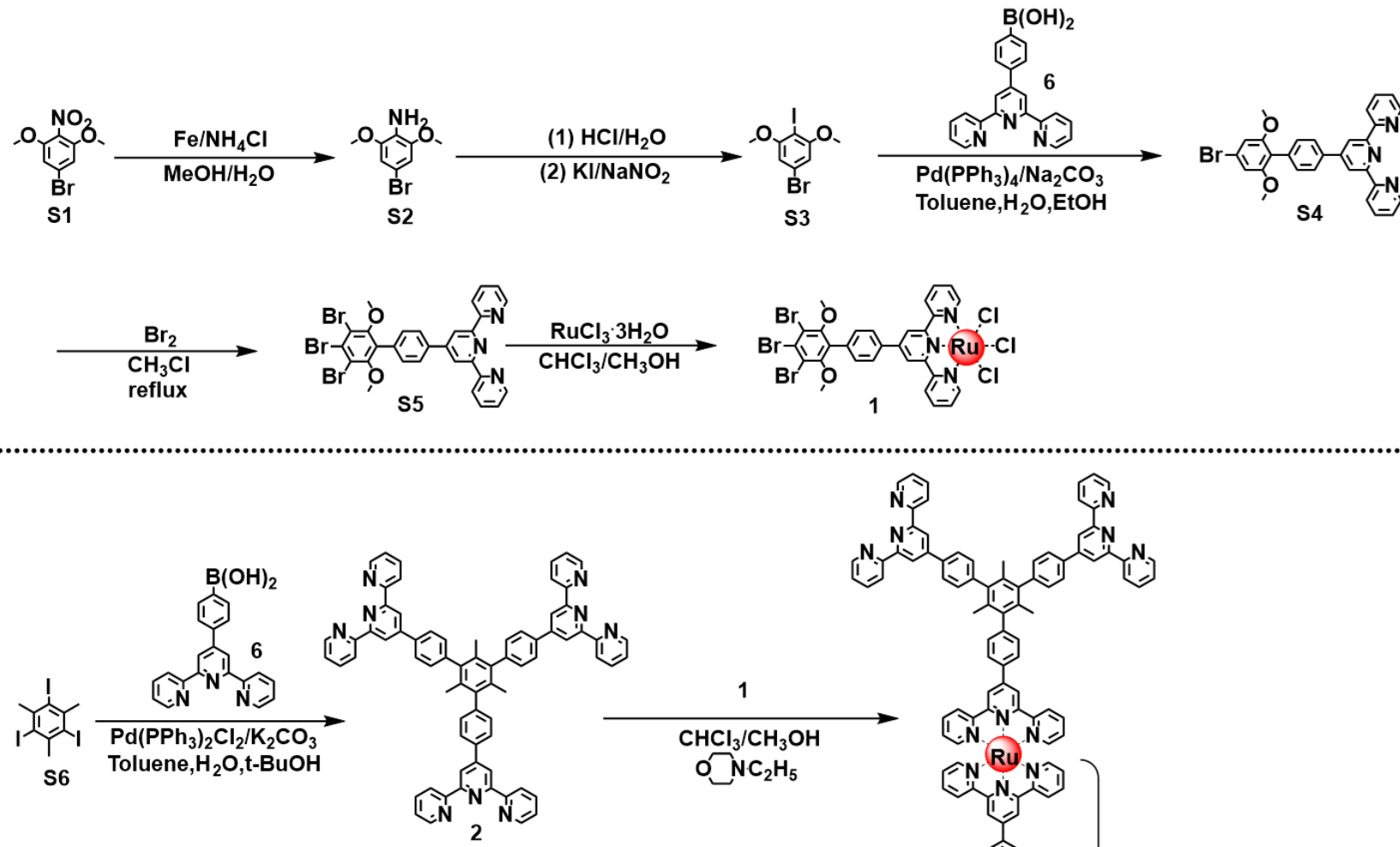

S6

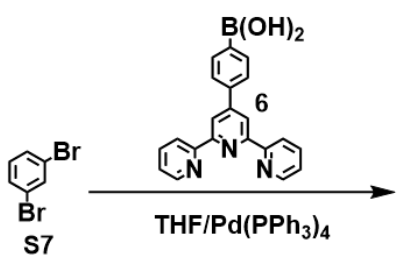<smiles>Cc1cc(Br)ccc1Br</smiles>

\$8

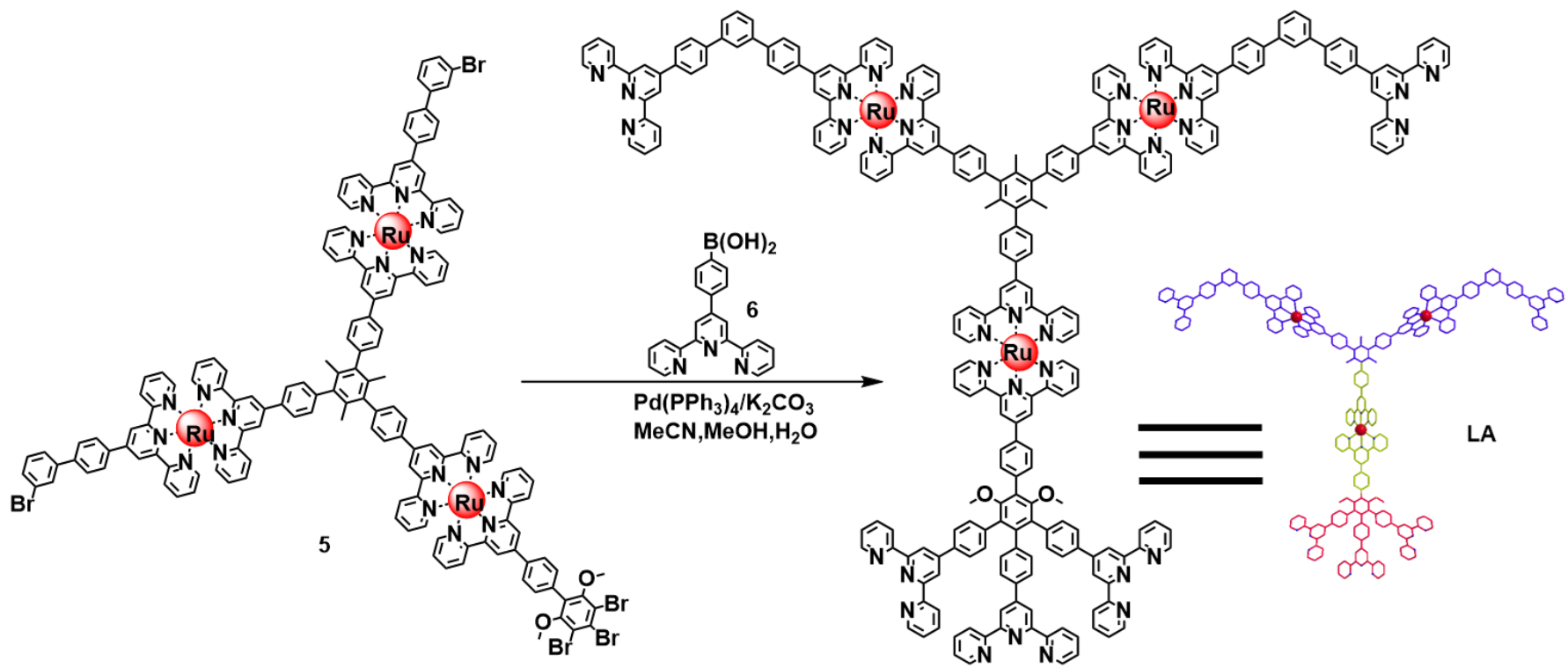

Scheme S1. Synthetic route to the key metalloorganic Ligand LA. 


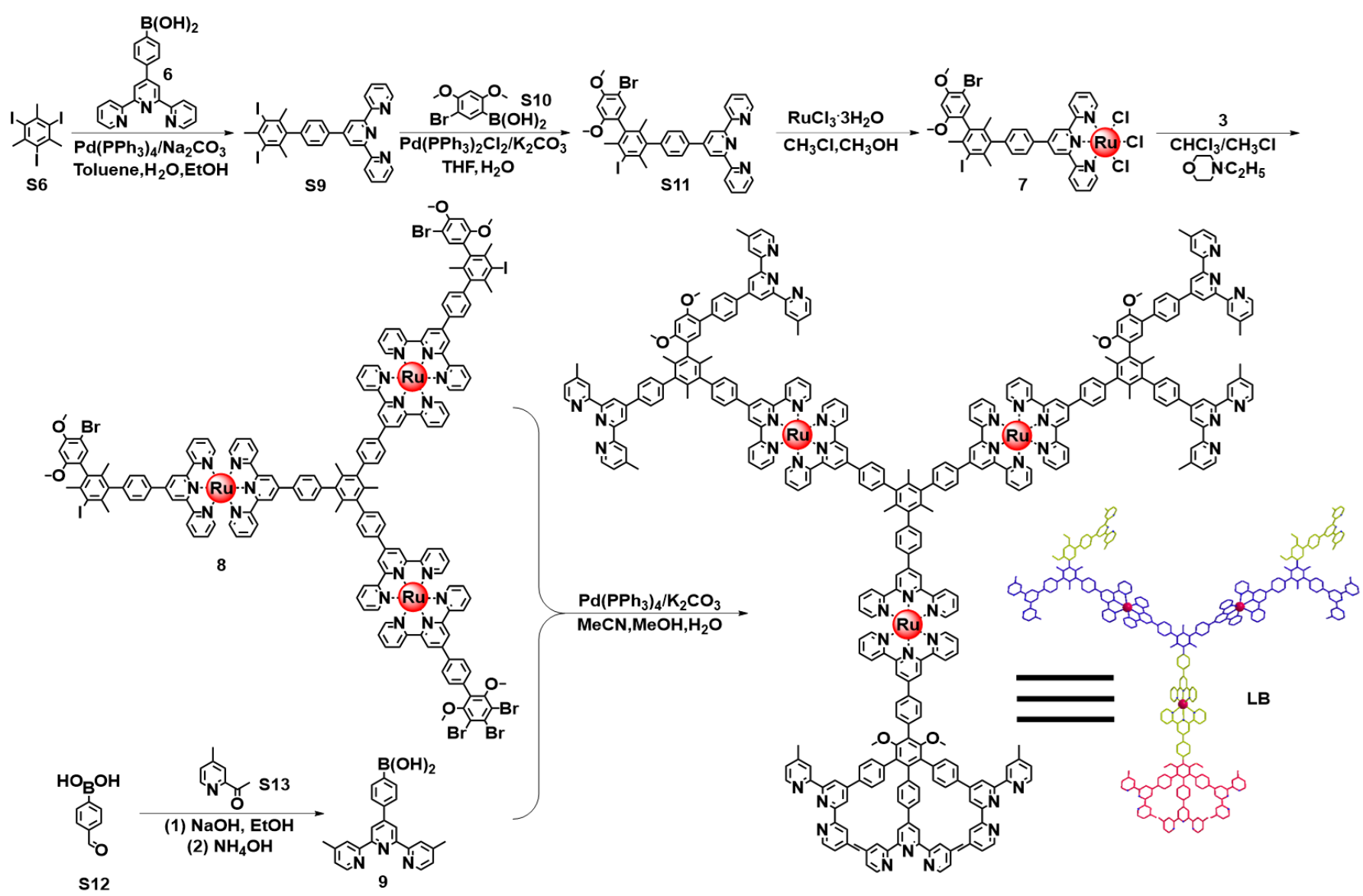

Scheme S2: Synthetic route to organic Ligand LB.

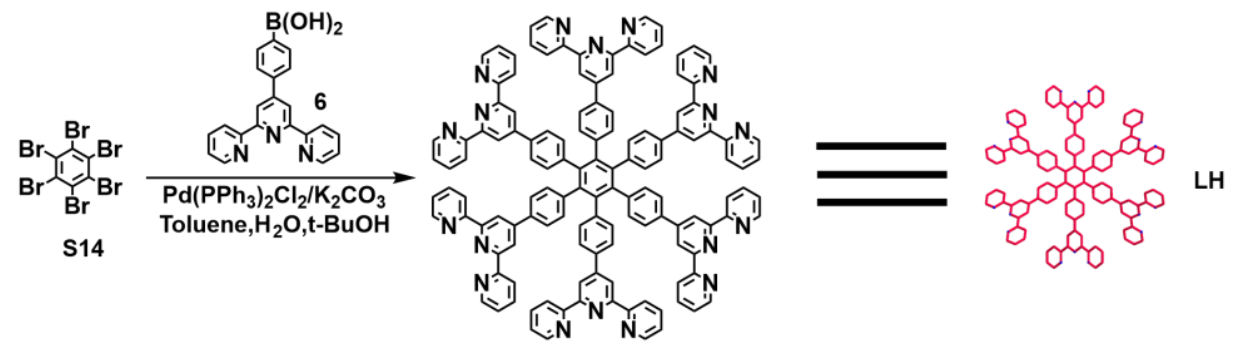

Scheme S3: Synthetic route to organic Ligand LH. ${ }^{\mathrm{S} 1}$ 


\section{Self-Assembly of Hexagon-Fused Wheel W1 and Prismatic Lamellar Structure P1}

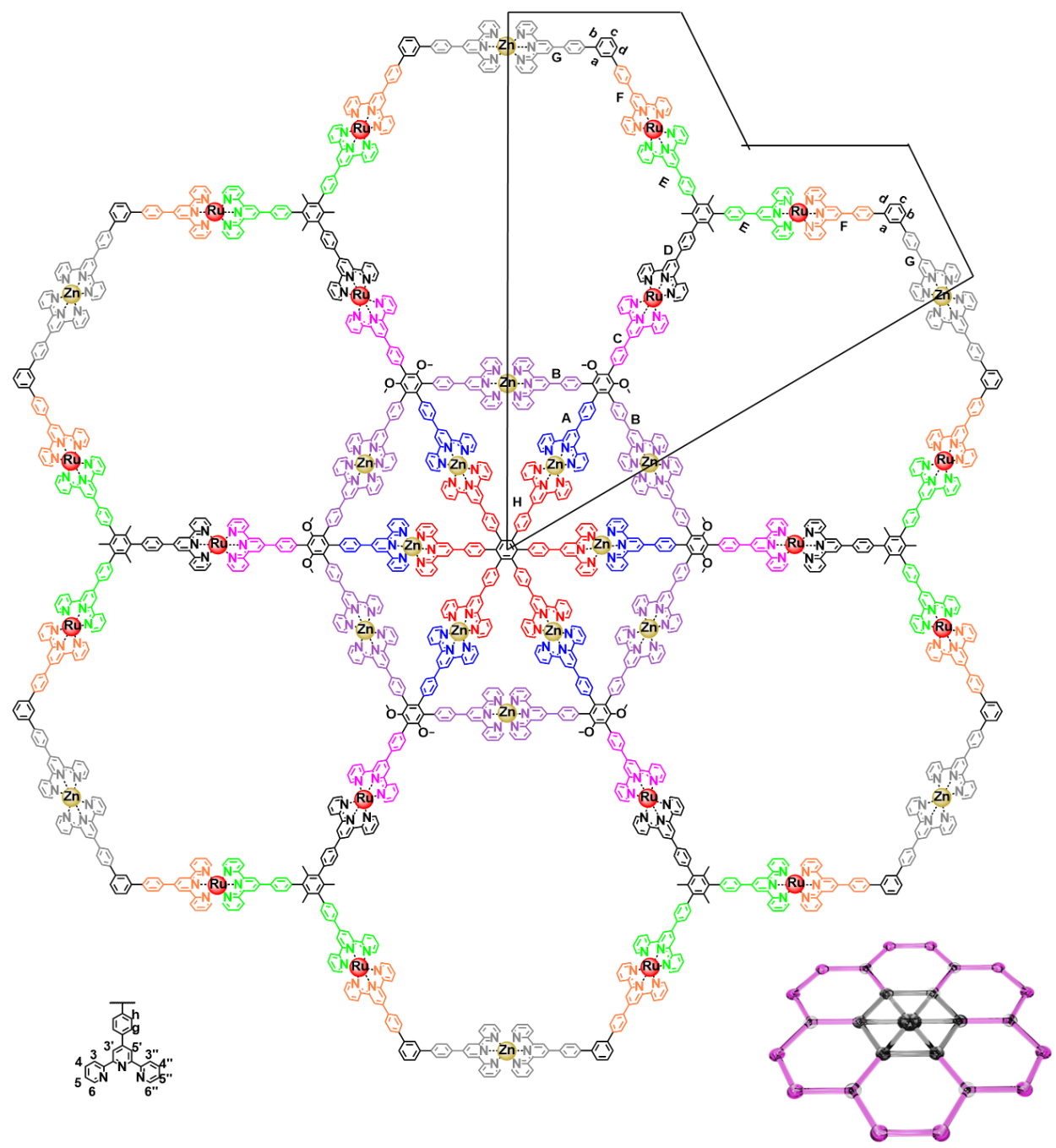

Hexagon-fused wheel W1:

To a flask containing $\mathbf{L A}(3.3 \mathrm{mg}, 0.54 \mu \mathrm{mol})$, ligand $\mathbf{L H}(0.2 \mathrm{mg}, 0.09 \mu \mathrm{mol})$, and $\mathrm{Zn}\left(\mathrm{NO}_{3}\right)_{2} \cdot 6 \mathrm{H}_{2} \mathrm{O}(0.5 \mathrm{mg}, 1.70 \mu \mathrm{mol})$, a solvent mixture of $\mathrm{CH}_{3} \mathrm{CN} / \mathrm{CHCl}_{3} / \mathrm{MeOH}(30 \mathrm{~mL}, \mathrm{~V}: \mathrm{V}: \mathrm{V}=2: 1: 1)$ was added. The mixture was refluxed for $24 \mathrm{~h}$. After cooling to ambient temperature, excess $\mathrm{LiN}\left(\mathrm{SO}_{2} \mathrm{CF}_{3}\right)_{2}$ in $\mathrm{MeOH}$ was added to generate red precipitates, which were filtered and washed with $\mathrm{MeOH}$ to afford a red solid: $4.3 \mathrm{mg}, 94 \% .{ }^{1} \mathrm{H}$ NMR (500 MHz, CD 3 CN) $\delta$ 9.21-9.12 (m, 108H, A-tpy-

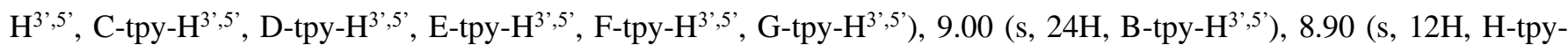

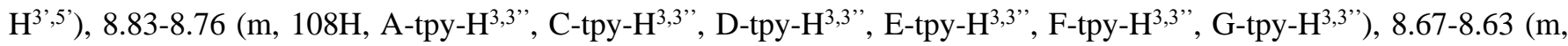

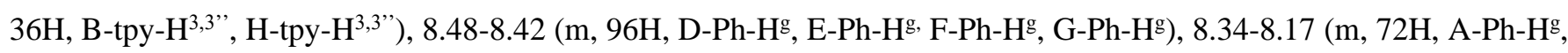

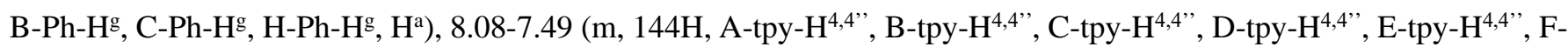

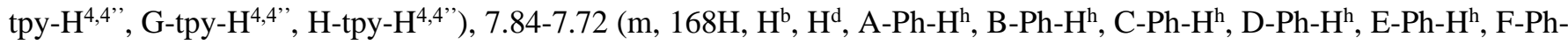

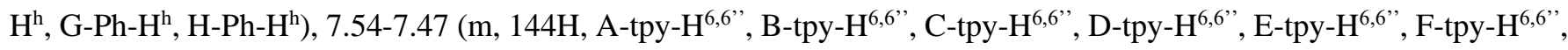

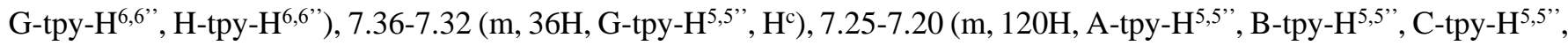




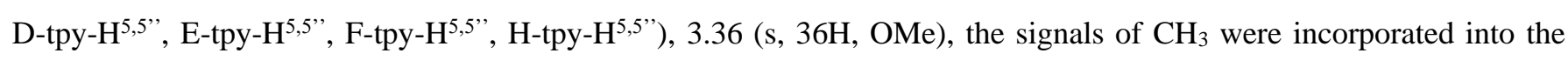
signals of $\mathrm{CD}_{3} \mathrm{CN}$. ESI-MS (m/z): $1999.18\left[\mathrm{M}-21 \mathrm{~N}\left(\mathrm{SO}_{2} \mathrm{CF}_{3}\right)_{2}{ }^{-}\right]^{21+}(\mathrm{calcd} \mathrm{m} / \mathrm{z}: 1998.97), 1895.16\left[\mathrm{M}-22 \mathrm{~N}\left(\mathrm{SO}_{2} \mathrm{CF}_{3}\right)_{2}{ }^{-}\right]^{22+}$ (calcd m/z: 1895.38), 1800.24 [M-23N( $\left.\left.\mathrm{SO}_{2} \mathrm{CF}_{3}\right)_{2}{ }^{-}\right]^{23+}(\mathrm{calcd} \mathrm{m} / \mathrm{z}: 1800.81), 1895.16\left[\mathrm{M}-22 \mathrm{~N}\left(\mathrm{SO}_{2} \mathrm{CF}_{3}\right)_{2}{ }^{-}\right]^{22+}(\mathrm{calcd} \mathrm{m} / \mathrm{z}$ : 1895.38), $1714.52\left[\mathrm{M}-24 \mathrm{~N}\left(\mathrm{SO}_{2} \mathrm{CF}_{3}\right)_{2}{ }^{-}\right]^{24+}(\mathrm{calcd} \mathrm{m} / \mathrm{z}: 1714.07), 1634.29\left[\mathrm{M}-25 \mathrm{~N}\left(\mathrm{SO}_{2} \mathrm{CF}_{3}\right)_{2}{ }^{-}\right]^{25+}(\mathrm{calcd} \mathrm{m} / \mathrm{z}: 1634.31)$, $1560.34\left[\mathrm{M}-26 \mathrm{~N}\left(\mathrm{SO}_{2} \mathrm{CF}_{3}\right)_{2}{ }^{-}\right]^{26+}\left(\right.$ calcd m/z: 1560.68), $1492.42\left[\mathrm{M}-27 \mathrm{~N}\left(\mathrm{SO}_{2} \mathrm{CF}_{3}\right)_{2}{ }^{-}\right]^{27+}(\mathrm{calcd} \mathrm{m} / \mathrm{z}: 1492.51), 1429.17[\mathrm{M}-$ $\left.28 \mathrm{~N}\left(\mathrm{SO}_{2} \mathrm{CF}_{3}\right)_{2}{ }^{-}\right]^{28+}$ (calcd m/z: 1429.21), $1370.37\left[\mathrm{M}-29 \mathrm{~N}\left(\mathrm{SO}_{2} \mathrm{CF}_{3}\right)_{2}{ }^{-}\right]^{29+}$ (calcd m/z: 1370.24), 1315.34 [M-

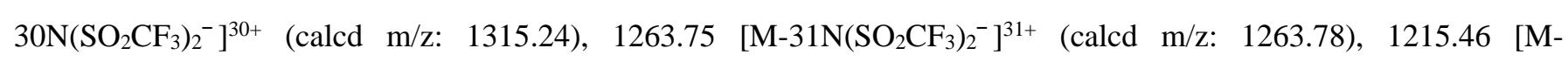

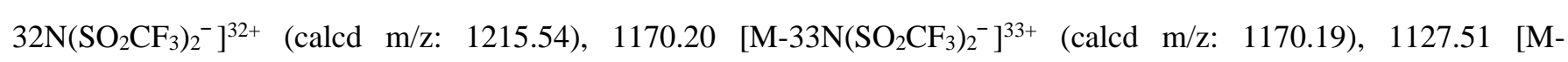
$\left.34 \mathrm{~N}\left(\mathrm{SO}_{2} \mathrm{CF}_{3}\right)_{2}{ }^{-}\right]^{34+}$ (calcd m/z: 1127.54), $1087.27\left[\mathrm{M}-35 \mathrm{~N}\left(\mathrm{SO}_{2} \mathrm{CF}_{3}\right)_{2}{ }^{-}\right]^{35+}$ (calcd m/z: 1087.33$), 1049.39$ [M$\left.36 \mathrm{~N}\left(\mathrm{SO}_{2} \mathrm{CF}_{3}\right)_{2}^{-}\right]^{36+}($ calcd m/z: 1049.35).

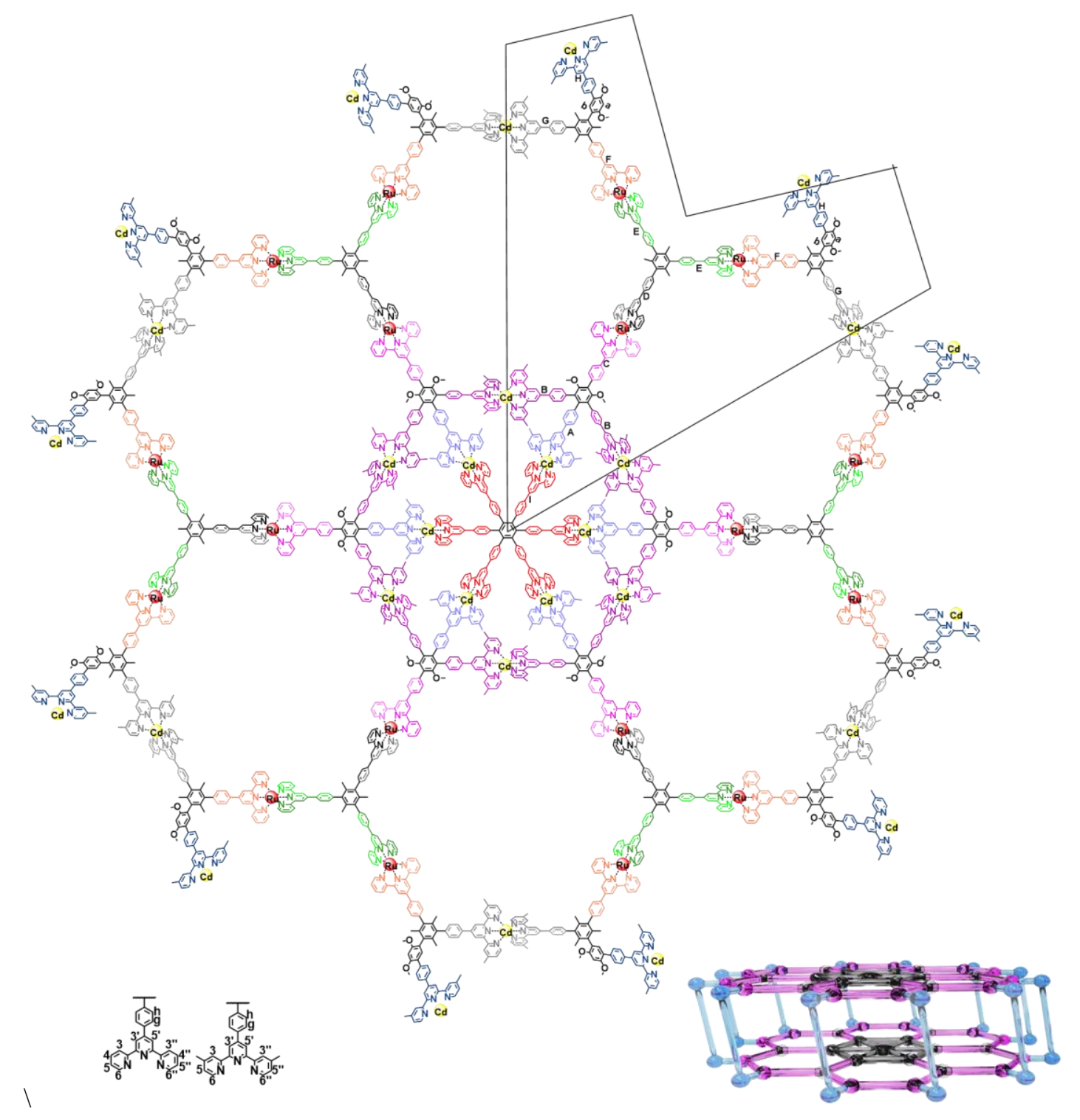


Prismatic lamellar structure P1:

To a flask containing $\mathbf{L B}(4.8 \mathrm{mg}, 0.69 \mu \mathrm{mol})$, ligand $\mathbf{L H}(0.2 \mathrm{mg}, 0.12 \mu \mathrm{mol})$, and $\mathrm{Cd}\left(\mathrm{NO}_{3}\right)_{2} \cdot 4 \mathrm{H}_{2} \mathrm{O}(0.8 \mathrm{mg}, 2.70 \mu \mathrm{mol})$, a solvent mixture of $\mathrm{CH}_{3} \mathrm{CN} / \mathrm{CHCl}_{3} / \mathrm{MeOH}(40 \mathrm{~mL}, \mathrm{~V}: \mathrm{V}: \mathrm{V}=2: 1: 1)$ was added. The mixture was refluxed for $24 \mathrm{~h}$. After cooling to ambient temperature, excess $\mathrm{LiN}\left(\mathrm{SO}_{2} \mathrm{CF}_{3}\right)_{2}$ in $\mathrm{MeOH}$ was added to generate red precipitates, which were filtered and washed with $\mathrm{MeOH}$ to afford a red solid: $6.2 \mathrm{mg}, 90 \% .{ }^{1} \mathrm{H} \mathrm{NMR}\left(500 \mathrm{MHz}, \mathrm{CD}_{3} \mathrm{CN} / \mathrm{DMSO}(2 / 1)\right) \delta$ 9.37-9.31 (m,

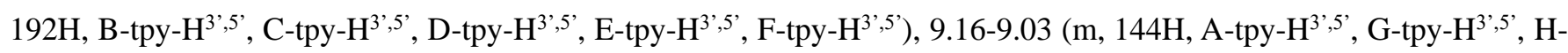

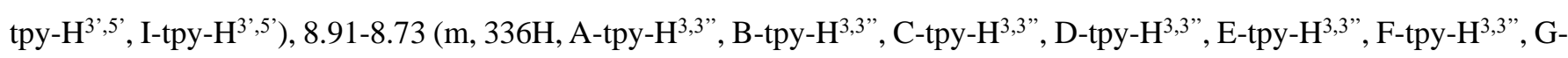

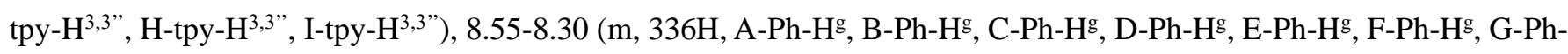

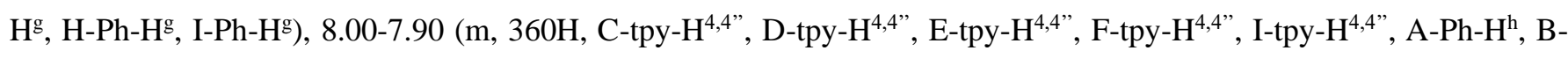

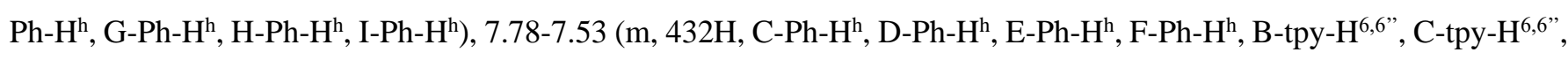

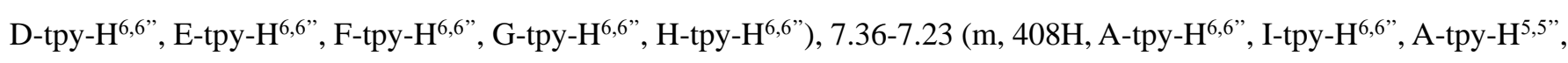

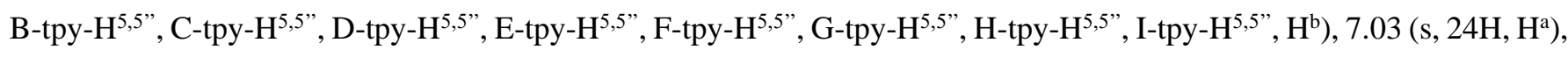
4.04-3.99 (m, 144H, OMe), 3.33 (s, 72H, OMe), the signals of $\mathrm{CH}_{3}$ were incorporated into the signals of $\mathrm{CD}_{3} \mathrm{CN}$. ESI-MS (m/z): $3243.00\left[\mathrm{M}-34 \mathrm{~N}\left(\mathrm{SO}_{2} \mathrm{CF}_{3}\right)_{2}{ }^{-}+4 \mathrm{DMF}\right]^{34+}(\mathrm{calcd} \mathrm{m} / \mathrm{z}: 3243.04), 3142.39\left[\mathrm{M}-35 \mathrm{~N}\left(\mathrm{SO}_{2} \mathrm{CF}_{3}\right)_{2}{ }^{-}+4 \mathrm{DMF}\right]^{35+}(\mathrm{calcd} \mathrm{m} / \mathrm{z}:$ 3142.44), $3047.33\left[\mathrm{M}-36 \mathrm{~N}\left(\mathrm{SO}_{2} \mathrm{CF}_{3}\right)_{2}{ }^{-}+4 \mathrm{DMF}\right]^{36+}(\mathrm{calcd} \mathrm{m} / \mathrm{z}: 3047.35), 2957.59\left[\mathrm{M}-37 \mathrm{~N}\left(\mathrm{SO}_{2} \mathrm{CF}_{3}\right)_{2}{ }^{-}+4 \mathrm{DMF}\right]^{37+}(\mathrm{calcd}$ $\mathrm{m} / \mathrm{z}: 2957.55), 2872.25\left[\mathrm{M}-38 \mathrm{~N}\left(\mathrm{SO}_{2} \mathrm{CF}_{3}\right)_{2}{ }^{-}+4 \mathrm{DMF}\right]^{38+}\left(\right.$ calcd m/z: 2875.30), $2791.46\left[\mathrm{M}-39 \mathrm{~N}\left(\mathrm{SO}_{2} \mathrm{CF}_{3}\right)_{2}{ }^{-}+4 \mathrm{DMF}\right]^{39+}$ $\left.\begin{array}{llllllllll}\text { calcd } & \mathrm{m} / \mathrm{z}: & 2791.48\end{array}\right), \quad 2714.60 \quad\left[\mathrm{M}-40 \mathrm{~N}\left(\mathrm{SO}_{2} \mathrm{CF}_{3}\right)_{2}{ }^{-}+4 \mathrm{DMF}\right]^{40+} \quad($ calcd $\mathrm{m} / \mathrm{z}: \quad 2714.66), \quad 2641.55 \quad[\mathrm{M}-$ $\left.41 \mathrm{~N}\left(\mathrm{SO}_{2} \mathrm{CF}_{3}\right)_{2}{ }^{-}+4 \mathrm{DMF}\right]^{41+}$ (calcd m/z: 2641.58), $2571.98\left[\mathrm{M}-42 \mathrm{~N}\left(\mathrm{SO}_{2} \mathrm{CF}_{3}\right)_{2}{ }^{-}+4 \mathrm{DMF}\right]^{42+}$ (calcd m/z: 2572.02), 2505.43 $\left[\mathrm{M}-43 \mathrm{~N}\left(\mathrm{SO}_{2} \mathrm{CF}_{3}\right)_{2}{ }^{-}+4 \mathrm{DMF}\right]^{43+}\left(\right.$ calcd m/z:2505.42), $2442.52\left[\mathrm{M}-44 \mathrm{~N}\left(\mathrm{SO}_{2} \mathrm{CF}_{3}\right)_{2}{ }^{-}+4 \mathrm{DMF}\right]^{44+}$ (calcd m/z: 2442.49),

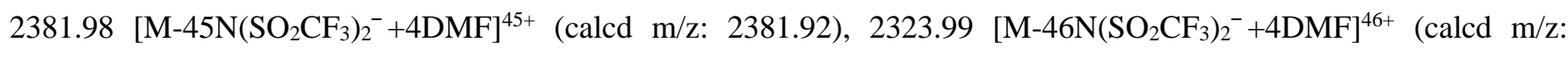
2324.03), $2268.64\left[\mathrm{M}-47 \mathrm{~N}\left(\mathrm{SO}_{2} \mathrm{CF}_{3}\right)_{2}{ }^{-}+4 \mathrm{DMF}\right]^{47+}(\mathrm{calcd} \mathrm{m} / \mathrm{z}: 2268.63), 2215.50\left[\mathrm{M}-48 \mathrm{~N}\left(\mathrm{SO}_{2} \mathrm{CF}_{3}\right)_{2}{ }^{-}+4 \mathrm{DMF}\right]^{48+}(\mathrm{calcd}$ $\mathrm{m} / \mathrm{z}: 2215.47), 2164.54\left[\mathrm{M}-49 \mathrm{~N}\left(\mathrm{SO}_{2} \mathrm{CF}_{3}\right)_{2}{ }^{-}+4 \mathrm{DMF}\right]^{49+}(\mathrm{calcd} \mathrm{m} / \mathrm{z}: 2164.58), 2115.80\left[\mathrm{M}-50 \mathrm{~N}\left(\mathrm{SO}_{2} \mathrm{CF}_{3}\right)_{2}{ }^{-}+4 \mathrm{DMF}\right]^{50+}$ $\left.\begin{array}{llllllllll}\text { calcd } & \mathrm{m} / \mathrm{z}: & 2115.72), & 2068.68 & {\left[\mathrm{M}-51 \mathrm{~N}\left(\mathrm{SO}_{2} \mathrm{CF}_{3}\right)_{2}{ }^{-}+4 \mathrm{DMF}\right]^{51+} \quad(\text { calcd }} & \mathrm{m} / \mathrm{z}: & 2068.70\end{array}\right), \quad 2023.55 \quad[\mathrm{M}-$ $\left.52 \mathrm{~N}\left(\mathrm{SO}_{2} \mathrm{CF}_{3}\right)_{2}{ }^{-}+4 \mathrm{DMF}\right]^{52+}(\mathrm{calcd} \mathrm{m} / \mathrm{z}: 2023.61)$.

\section{Synthesis of the ligands, compounds and complexes.}

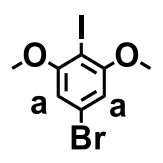

Compound S3: 


\section{Electronic Supporting Information}

A $250 \mathrm{~mL}$, three-necked, round-bottomed flask fitted with an electromagnetic stirrer was charged with $1.30 \mathrm{~g}$ of compound S2 $(5.60 \mathrm{mmol})$ and $12 \mathrm{~mL}$ of hydrochloric acid $(6 \mathrm{~mol} / \mathrm{L})$. This solution was then cooled in a beaker with an ice-salt mixture. With vigorous stirring, a solution of sodium nitrite $(460.0 \mathrm{mg}, 6.67 \mathrm{mmol})$ was added (temp. $\left.5^{\circ} \mathrm{C}\right)$. Two hours later, the diazonium salt was added into a solution of potassium iodide $(2.30 \mathrm{~g}, 13.86 \mathrm{mmol})$. The reaction mixture was stirred for about $3 \mathrm{~h}$. The crude product was filtered out, washed with water, and purified by silica-gel column chromatography using petroleum ether as the eluent. Recrystallization from ethanol afforded white solid: $1.15 \mathrm{~g}, 60 \% .{ }^{1} \mathrm{H}$ NMR (500 MHz, $\left.\mathrm{CDCl}_{3}\right) \delta 6.67\left(\mathrm{~s}, 2 \mathrm{H}, \mathrm{H}^{\mathrm{a}}\right), 3.90(\mathrm{~s}, 6 \mathrm{H}, \mathrm{OMe}) .{ }^{13} \mathrm{C} \mathrm{NMR}\left(101 \mathrm{MHz}, \mathrm{CDCl}_{3}\right) \delta 159.90,123.44,107.86$, $104.14,56.80$.

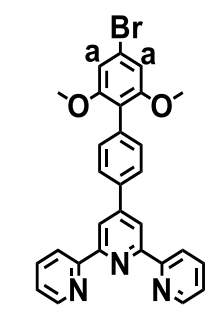

Compound S4:

To a Schlenk flask containing compound $\mathbf{S 3}(888.0 \mathrm{mg}, 2.59 \mathrm{mmol})$, compound 6 (869.0 mg, $\left.2.46 \mathrm{mmol}), \mathrm{Pd}_{(\mathrm{PPh}}\right)_{4}(70.0$ $\mathrm{mg}, 0.06 \mathrm{mmol})$ and $\mathrm{Na}_{2} \mathrm{CO}_{3}(1.40 \mathrm{~g}, 13.21 \mathrm{mmol})$ were added. After the removal of air and back-filled with argon, $20 \mathrm{~mL}$ of toluene, $5 \mathrm{~mL}$ of water and $5 \mathrm{~mL}$ of EtOH were added. After stirring at $85^{\circ} \mathrm{C}$ for $12 \mathrm{~h}$, the mixture was cooled to $25^{\circ} \mathrm{C}$. The aqueous layer was extracted with DCM, and then the combined organic phase was washed with brine and dried with $\mathrm{MgSO}_{4}$. The solvents were then removed under reduced pressure. The residue was purified by column chromatography $\left(\mathrm{Al}_{2} \mathrm{O}_{3}\right)$, eluting with chloroform to afford compound $\mathbf{S} 4$ as white solid $(1.05 \mathrm{~g}, 77 \%) .{ }^{1} \mathrm{H}$ NMR $\left(500 \mathrm{MHz}, \mathrm{CDCl}_{3}\right) \delta 8.82$

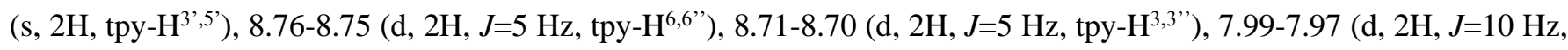

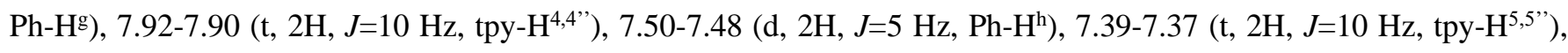
$6.85\left(\mathrm{~s}, 2 \mathrm{H}, \mathrm{H}^{\mathrm{a}}\right), 3.79$ (s, 6H, OMe). ${ }^{13} \mathrm{C} \mathrm{NMR}\left(126 \mathrm{MHz}, \mathrm{CDCl}_{3}\right) \delta 158.11,156.37,155.90,149.16,137.03,136.83$, 134.08, 131.43, 126.73, 123.76, 122.02, 121.34, 118.91, 118.01, 117.81, 108.13, 56.19. ESI-MS (524.42 calcd. For $\mathrm{C}_{29} \mathrm{H}_{22} \mathrm{~N}_{3} \mathrm{O}_{2} \mathrm{Br}$ ): m/z $526.13[\mathrm{M}+\mathrm{H}]^{+}$(calcd. m/z: 526.09). 


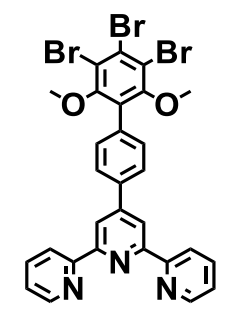

Compound S5:

To a solution of compound $\mathbf{S} 4(200.0 \mathrm{mg}, 0.38 \mathrm{mmol})$ in $\mathrm{CHCl}_{3}(30 \mathrm{~mL})$, a solution of $\mathrm{Br}_{2}(2 \mathrm{~mL}, 40.00 \mathrm{mmol})$ in $\mathrm{CHCl}_{3}$ $(5 \mathrm{~mL})$ was added dropwise. After refluxing for $24 \mathrm{~h}$, the mixture was washed by saturated $\mathrm{NaHSO}_{3}$ until colorless. The organic layer was dried (anhydrous $\mathrm{Na}_{2} \mathrm{SO}_{4}$ ), and then concentrated in vacuo to give compound $\mathbf{S 5}$, as light yellow solid: $210.7 \mathrm{mg}, 81 \% .{ }^{1} \mathrm{H}$ NMR $\left(500 \mathrm{MHz}, \mathrm{CDCl}_{3}\right) \delta 8.82\left(\mathrm{~s}, 2 \mathrm{H}, \mathrm{tpy}-\mathrm{H}^{3}, 5^{\prime}\right), 8.77-8.76$ (d, 2H, J=5 Hz, tpy-H $\mathrm{H}^{6,6}$ '), 8.73-8.72 (d,

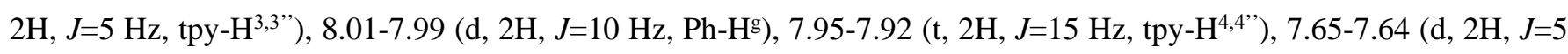
$\left.\mathrm{Hz}, \mathrm{Ph}-\mathrm{H}^{\mathrm{h}}\right), 7.41-7.39$ (t, 2H, J=10 Hz, tpy-H ${ }^{5,5}$ ), 3.43 (s, 6H, OMe). ${ }^{13} \mathrm{C}$ NMR (126 MHz, $\left.\mathrm{CDCl}_{3}\right) \delta 156.16,156.04$, $155.74,149.91,149.82,149.15,136.99,130.74,130.08,127.77,127.24,123.95,121.42,120.66,119.01,118.03,60.54$. ESI-MS (682.21 calcd. For $\left.\mathrm{C}_{29} \mathrm{H}_{20} \mathrm{~N}_{3} \mathrm{O}_{2} \mathrm{Br}_{3}\right)$ : m/z $683.96[\mathrm{M}+\mathrm{H}]^{+}$(calcd. m/z: 683.91).

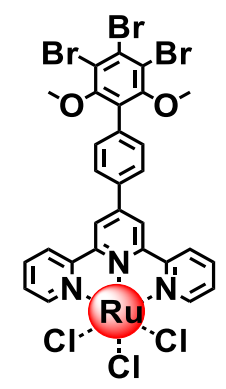

Compound 1:

To a solution of compound $\mathbf{S 5}(200.0 \mathrm{mg}, 0.29 \mathrm{mmol})$ and $\mathrm{RuCl}_{3} \cdot 3 \mathrm{H}_{2} \mathrm{O}(80.0 \mathrm{mg}, 0.31 \mathrm{mmol})$ in $\mathrm{CHCl}_{3} / \mathrm{MeOH}(30 \mathrm{~mL})$. The mixture was stirred at $72{ }^{\circ} \mathrm{C}$ for $24 \mathrm{~h}$. After cooling to ambient temperature, the precipitates were filtered and washed with $\mathrm{MeOH}$ to afford compound 1: $211.3 \mathrm{mg}, 81 \%$.

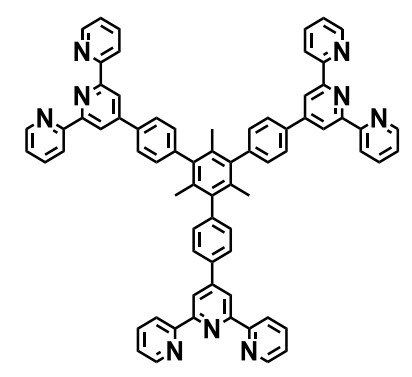

Compound 2: 


\section{Electronic Supporting Information}

Compound $\mathbf{S 6}(996.0 \mathrm{mg}, 2.00 \mathrm{mmol})$ and compound $\mathbf{6}(2.64 \mathrm{~g}, 7.20 \mathrm{mmol})$ was added to a $250 \mathrm{~mL}$ flask, then $\mathrm{K}_{2} \mathrm{CO}_{3}$ (2.49 g, $18.02 \mathrm{mmol}), 60 \mathrm{~mL}$ of toluene, $60 \mathrm{~mL}$ of water and $20 \mathrm{~mL}$ of t-butanol were added. After the removal of air and back-filled with argon, $\mathrm{Pd}\left(\mathrm{PPh}_{3}\right)_{2} \mathrm{Cl}_{2}(211.0 \mathrm{mg}, 0.30 \mathrm{mmol})$ were added. The mixture was stirred at $85^{\circ} \mathrm{C}$ under nitrogen for $48 \mathrm{~h}$, after cooled to ambient temperature, then concentrated in vacuo followed by column chromatography $\left(\mathrm{Al}_{2} \mathrm{O}_{3}\right)$, eluting with the mixture of petroleum ether and DCM to pure the product, as white solid: $1.79 \mathrm{~g}, 86 \% .{ }^{1} \mathrm{H} \mathrm{NMR}(500 \mathrm{MHz}$, $\left.\mathrm{CDCl}_{3}\right) \delta 8.84\left(\mathrm{~s}, 6 \mathrm{H}\right.$, tpy- $\left.^{3^{\prime}, 5^{\prime}}\right), 8.76-8.75\left(\mathrm{~d}, 6 \mathrm{H}, J=5 \mathrm{~Hz}\right.$, tpy- $\left.\mathrm{H}^{6,6^{\prime}}\right), 8.72-8.70\left(\mathrm{~d}, 6 \mathrm{H}, J=10 \mathrm{~Hz}\right.$, tpy- $\left.\mathrm{H}^{3,3 ”}\right), 8.04-8.03(\mathrm{~d}$, 6H, $J=5 \mathrm{~Hz}, \mathrm{Ph}-\mathrm{H}^{\mathrm{g}}$ ), 7.92-7.89 (t, 6H, $J=15 \mathrm{~Hz}, \mathrm{tpy}-\mathrm{H}^{4,4}$ )), 7.47-7.45 (d, 6H, $J=10 \mathrm{~Hz}, \mathrm{Ph}-\mathrm{H}^{\mathrm{h}}$ ), 7.39-7.36 (t, 6H, $J=15 \mathrm{~Hz}$, tpy-H ${ }^{5,5 ")}, 1.88\left(\mathrm{~s}, 9 \mathrm{H}, \mathrm{CH}_{3}\right) .{ }^{13} \mathrm{C}$ NMR $\left(126 \mathrm{MHz}, \mathrm{CDCl}_{3}\right) \delta 156.31,155.95,150.27,149.16,142.93,139.46,136.86$, 133.37, 130.06, 127.65, 123.80, 121.34, 118.99, 19.56. ESI-MS (1042.26 calcd. For $\left.\mathrm{C}_{72} \mathrm{H}_{51} \mathrm{~N}_{9}\right): \mathrm{m} / \mathrm{z} 1043.44[\mathrm{M}+\mathrm{H}]^{+}$ (calcd. m/z: 1043.43).

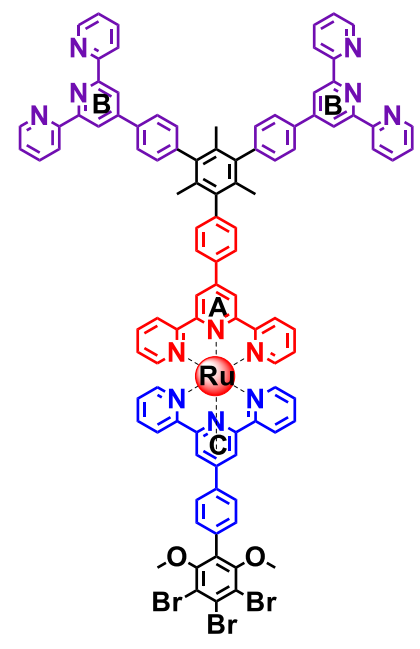

Complex 3:

To a flask containing a mixture of compound $2(164.0 \mathrm{mg}, 0.16 \mathrm{mmol})$ and compound 1 (70.0 $\mathrm{mg}, 0.08 \mathrm{mmol}), \mathrm{MeOH}$ $(150 \mathrm{~mL}), \mathrm{CHCl}_{3}(150 \mathrm{~mL})$, and drops of $\mathrm{N}$-ethylmorpholine were added. The mixture was stirred at $80^{\circ} \mathrm{C}$ for $20 \mathrm{~h}$. After cooling to ambient temperature, the solvent was evaporated in vacuo and the residue was subjected to column chromatography $\left(\mathrm{Al}_{2} \mathrm{O}_{3}, \mathrm{CH}_{2} \mathrm{Cl}_{2} / \mathrm{MeOH}\right)$ to afford complex 3 as red solid: $94.0 \mathrm{mg}, 63 \% .{ }^{1} \mathrm{H} \mathrm{NMR}\left(500 \mathrm{MHz}, \mathrm{CD}_{3} \mathrm{Cl}\right) \delta$

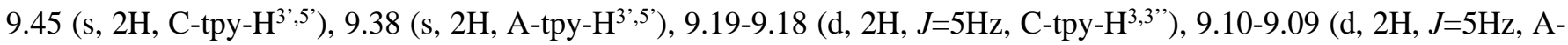

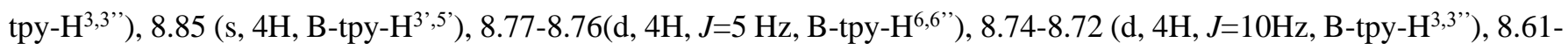
$8.59\left(\mathrm{~d}, 2 \mathrm{H}, J=10 \mathrm{~Hz}, \mathrm{C}-\mathrm{Ph}-\mathrm{H}^{\mathrm{g}}\right), 8.56-8.54\left(\mathrm{~d}, 2 \mathrm{H}, J=10 \mathrm{~Hz}, \mathrm{~A}-\mathrm{Ph}-\mathrm{H}^{\mathrm{g}}\right), 8.05-8.03$ (d, 4H, $\left.J=10 \mathrm{~Hz}, \mathrm{~B}-\mathrm{Ph}-\mathrm{H}^{\mathrm{g}}\right), 8.01-7.98$

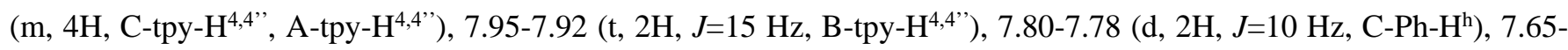

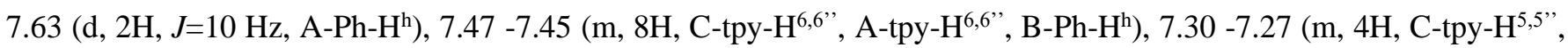


A-tpy-H ${ }^{5,5 ”}$ ), 3.49 (s, 6H, OMe), 1.88 (s, 9H, Me). $\left.{ }^{13} \mathrm{C} \mathrm{NMR} \mathrm{(101} \mathrm{MHz,} \mathrm{CDCl}_{3}\right) \delta$ 158.05, 155.98, 155.75, 155.19, 155.10, $151.87,151.78,150.20,148.95,144.85,142.85,139.52$, 138.98, 138.51, 137.21, 136.80, 133.11, 131.44, 130.06, 128.21, 128.04, 127.68, 125.90, 125.62, 123.99, 122.30, 122.11, 121.50, 119.06, 118.09, 60.89, 29.71, 19.68. ESI-MS (1896.44 calcd. For $\mathrm{C}_{101} \mathrm{H}_{71} \mathrm{Br}_{3} \mathrm{Cl}_{2} \mathrm{~N}_{12} \mathrm{O}_{2} \mathrm{Ru}$ ): m/z $1861.21\left[\mathrm{M}-\mathrm{Cl}^{-}\right]^{1+}$ (calcd. m/z: 1861.20) and $913.14\left[\mathrm{M}-2 \mathrm{Cl}^{-}\right]^{2+}$ (calcd. m/z: 913.12).

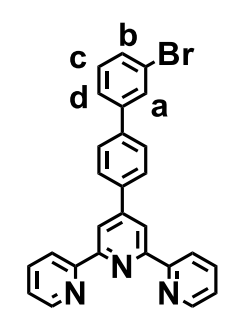

Compound S8:

Compound $\mathbf{S} 7(1.70 \mathrm{~g}, 7.21 \mathrm{mmol})$ and compound $\mathbf{6}(1.77 \mathrm{~g}, 5.01 \mathrm{mmol})$ was added to a $250 \mathrm{~mL}$ flask, then THF $(150 \mathrm{~mL})$ and $\mathrm{NaOH}(600.0 \mathrm{mg}, 15.00 \mathrm{mmol})$ in $15 \mathrm{~mL}$ of water was added. The system was degassed for $10 \mathrm{~min}$, and $\mathrm{Pd}\left(\mathrm{PPh}_{3}\right)_{4}$ $(130.0 \mathrm{mg}, 0.11 \mathrm{mmol})$ as the catalyst was added. The mixture was stirred at $85^{\circ} \mathrm{C}$ under nitrogen for $12 \mathrm{~h}$, after cooled to ambient temperature, then concentrated in vacuo followed by column chromatography $\left(\mathrm{Al}_{2} \mathrm{O}_{3}\right)$, eluting with the mixture of petroleum ether and $\mathrm{CH}_{2} \mathrm{Cl}_{2}$ to pure the product, as white solid: $1.86 \mathrm{~g}, 80 \% .{ }^{1} \mathrm{H} \mathrm{NMR}\left(500 \mathrm{MHz}, \mathrm{CDCl}_{3}\right) \delta 8.80(\mathrm{~s}, 2 \mathrm{H}$,

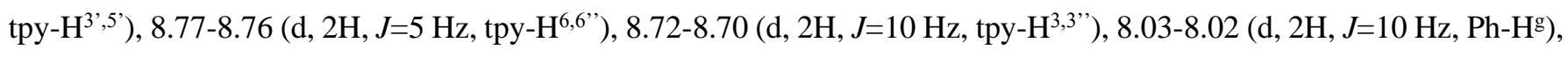

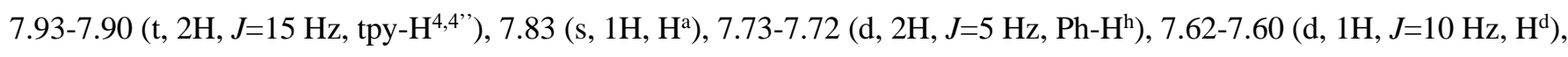
7.54-7.52 (d, $\left.1 \mathrm{H}, J=10 \mathrm{~Hz}, \mathrm{H}^{\mathrm{b}}\right), 7.40-7.37$ (t, $2 \mathrm{H}, J=15 \mathrm{~Hz}$, tpy-H ${ }^{5,5}$ "), 7.38-7.35 (t, $\left.1 \mathrm{H}, J=15 \mathrm{~Hz}, \mathrm{H}^{\mathrm{c}}\right) .{ }^{13} \mathrm{C} \mathrm{NMR}(126 \mathrm{MHz}$, $\left.\mathrm{CDCl}_{3}\right) \delta 156.24,156.23,155.87,155.86,150.29,149.09,142.95,142.93,136.94,136.86,139.45,133.37,130.06,130.01$, 127.65, 123.83, 121.37, 119.04. ESI-MS (464.37 calcd. For $\mathrm{C}_{27} \mathrm{H}_{18} \mathrm{~N}_{3} \mathrm{Br}$ ): m/z 464.10 [M + H] (calcd. m/z: 464.08).

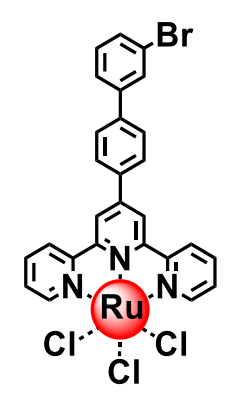

Compound 4:

To a solution of compound $\mathbf{S 8}(500.0 \mathrm{mg}, 1.08 \mathrm{mmol})$ and $\mathrm{RuCl}_{3} \cdot 3 \mathrm{H}_{2} \mathrm{O}(338.0 \mathrm{mg}, 1.29 \mathrm{mmol})$ in $\mathrm{CHCl}_{3} / \mathrm{MeOH}(60 \mathrm{~mL})$. The mixture was stirred at $72{ }^{\circ} \mathrm{C}$ for $24 \mathrm{~h}$. After cooling to ambient temperature, the precipitates were filtered and washed 
with $\mathrm{MeOH}$ to afford compound 4: $622.1 \mathrm{mg}, 86 \%$.

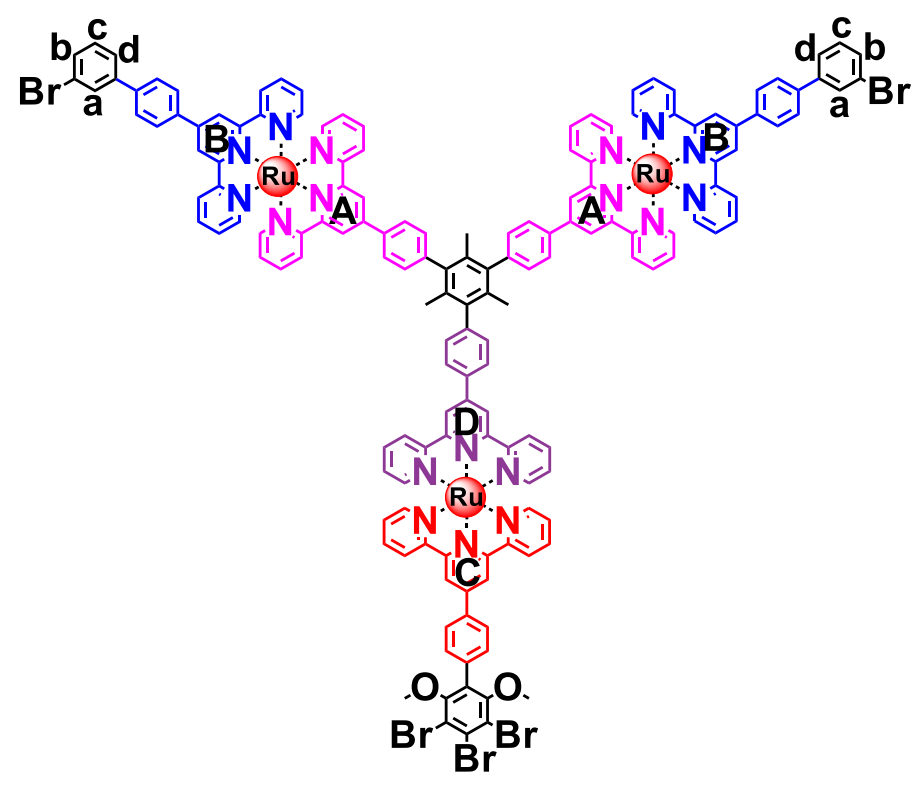

Complex 5:

To a flask containing a mixture of complex $3(43.0 \mathrm{mg}, 0.02 \mathrm{mmol})$ and compound 4 (47.0 mg, $0.07 \mathrm{mmol})$, MeOH (150 $\mathrm{mL}), \mathrm{CHCl}_{3}(150 \mathrm{~mL})$, and drops of $\mathrm{N}$-ethylmorpholine were added. The mixture was stirred at $80{ }^{\circ} \mathrm{C}$ for $20 \mathrm{~h}$. After cooling to ambient temperature, the solvent was evaporated in vacuo and the residue was subjected to column chromatography $\left(\mathrm{Al}_{2} \mathrm{O}_{3}, \mathrm{CH}_{2} \mathrm{Cl}_{2} / \mathrm{MeOH}\right)$ to afford complex 5 as red solid: $67.6 \mathrm{mg}, 94 \% .{ }^{1} \mathrm{H}$ NMR $(500 \mathrm{MHz}, \mathrm{MeOD}) \delta$

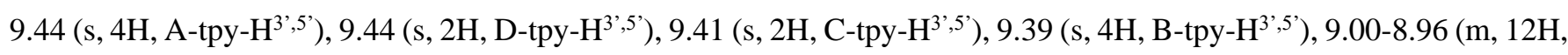

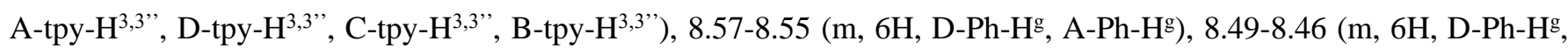

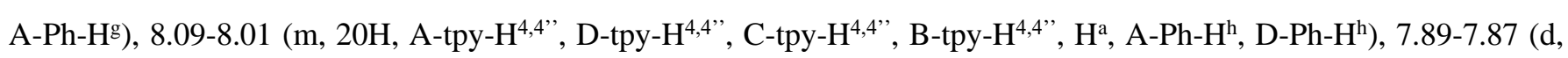

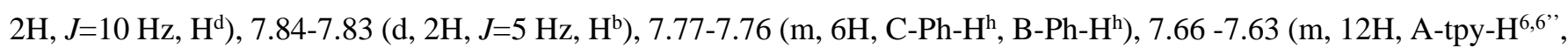

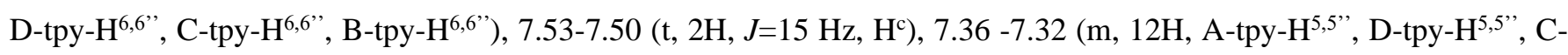
tpy-H ${ }^{5,5 ”}$, B-tpy-H $\mathrm{H}^{5,5 ”}$ ), 3.55 (s, 6H, OMe), 2.06 (s, 9H, Me). ${ }^{13} \mathrm{C}$ NMR (101 MHz, MeOD) $\delta$ 158.45, 155.92, 155.62, 152.01, 148.67, 148.23, 148.14, 144.37, 142.14, 141.50, 139.55, 138.08, 136.65, 136.20, 135.53, 134.88, 132.78, 131.39, $130.75,130.66,130.60,129.63,128.21,127.88,127.60,127.40,125.62,124.68,122.77,121.47,121.26,117.62,59.88$, 18.60, 16.98. ESI-MS (3169.12 calcd. For $\left.\mathrm{C}_{155} \mathrm{H}_{107} \mathrm{Br}_{5} \mathrm{Cl}_{6} \mathrm{~N}_{18} \mathrm{O}_{2} \mathrm{Ru}_{3}\right): \mathrm{m} / \mathrm{z} 1021.05$ [M-3Cl- $]^{3+}$ (calcd. m/z: 1021.03), $756.81\left[\mathrm{M}-4 \mathrm{Cl}^{-}\right]^{4+}\left(\right.$ calcd. m/z: 756.78) and $598.46\left[\mathrm{M}-5 \mathrm{Cl}^{-}\right]^{5+}($ calcd. m/z: 598.43). 


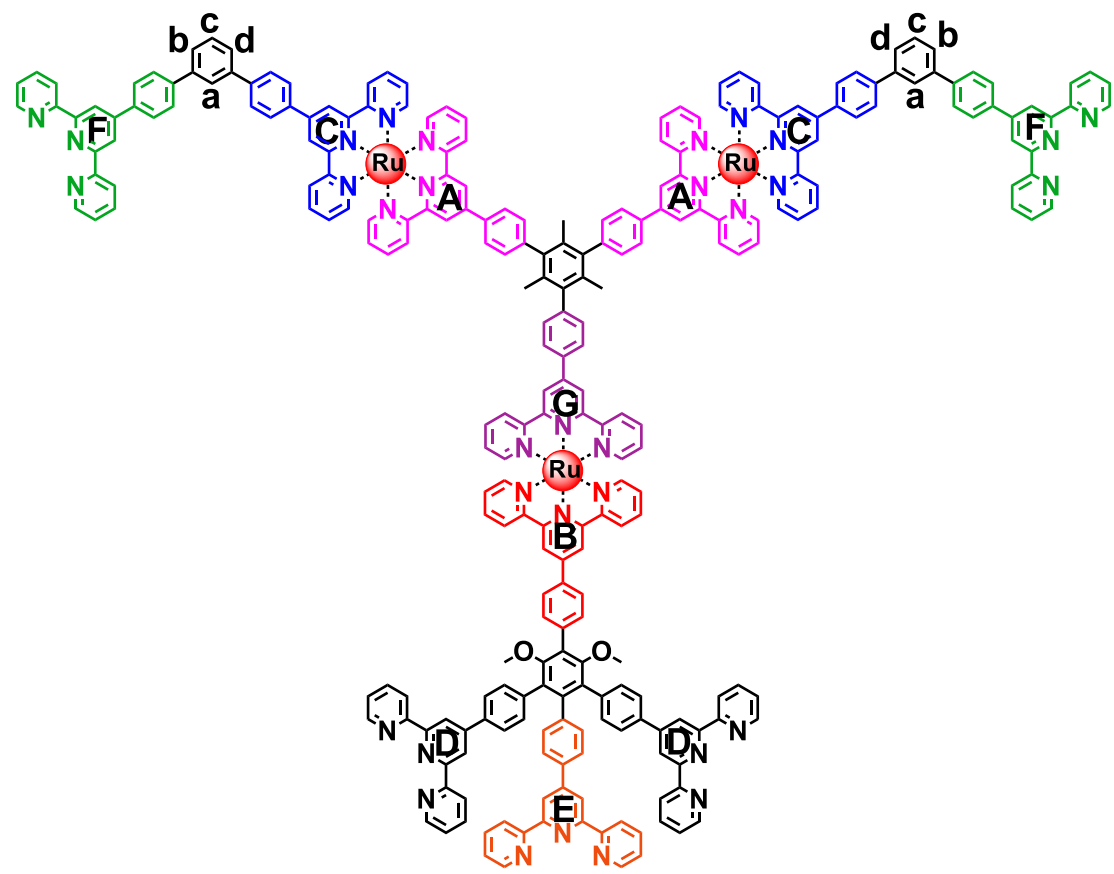

Ligand LA:

Complex $5(50.0 \mathrm{mg}, 0.02 \mathrm{mmol})$ and compound $6(226.0 \mathrm{mg}, 0.64 \mathrm{mmol})$ was added to a $100 \mathrm{~mL}$ flask, then acetonitrile $(45 \mathrm{~mL})$, methanol $(15 \mathrm{~mL})$ and $\mathrm{K}_{2} \mathrm{CO}_{3}(44.0 \mathrm{mg}, 0.32 \mathrm{mmol})$ in $0.3 \mathrm{~mL}$ of water was added. The system was degassed for $10 \mathrm{~min}$, and $\mathrm{Pd}\left(\mathrm{PPh}_{3}\right)_{4}(28.0 \mathrm{mg}, 0.02 \mathrm{mmol})$ as the catalyst was added. The mixture was stirred at $85{ }^{\circ} \mathrm{C}$ under nitrogen for $5 \mathrm{~d}$, after cooled to ambient temperature, then concentrated in vacuo followed by column chromatography $\left(\mathrm{Al}_{2} \mathrm{O}_{3}\right)$, eluting with the mixture of petroleum ether and $\mathrm{CH}_{2} \mathrm{Cl}_{2}$ to pure the product. The complex was counterion exchanged with $\mathrm{LiN}\left(\mathrm{SO}_{2} \mathrm{CF}_{3}\right)_{2}$ to give ligand $\mathbf{L A}$, as a red precipitate: $31.9 \mathrm{mg}, 35 \% .{ }^{1} \mathrm{H}$ NMR $\left(500 \mathrm{MHz}, \mathrm{CD}_{3} \mathrm{CN}\right) \delta 9.19-9.15(\mathrm{~m}, 12 \mathrm{H}$,

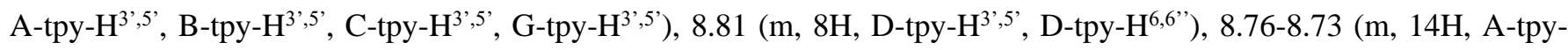

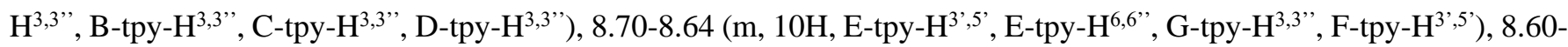

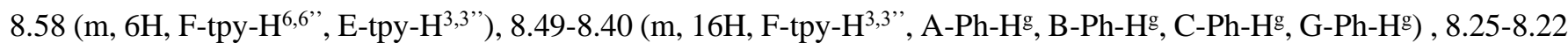

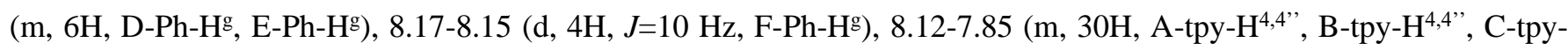

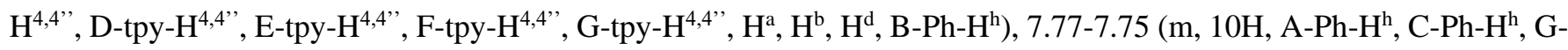

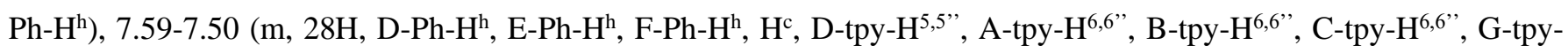

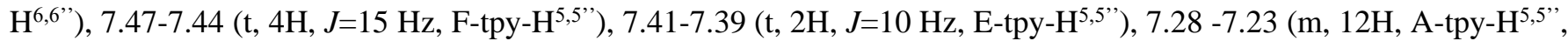

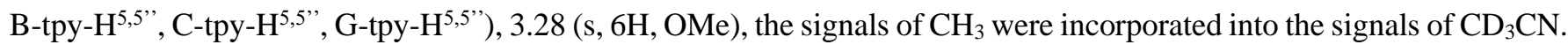
${ }^{13} \mathrm{C}$ NMR $\left(101 \mathrm{MHz}, \mathrm{CD}_{3} \mathrm{CN}\right) \delta 156.77,155.64,155.56,154.65,152.04,149.35,148.92,145.63,143.96,139.13,137.69$, $137.51,129.39,128.26,127.97,127.77,125.91,125.31,124.51,122.00,121.33,121.08,118.88,118.39,118.29,117.32$, 78.28, 57.64, 19.69. ESI-MS (5779.53 calcd. For $\left.\mathrm{C}_{272} \mathrm{H}_{177} \mathrm{~F}_{36} \mathrm{~N}_{39} \mathrm{O}_{26} \mathrm{Ru}_{3} \mathrm{~S}_{12}\right): \mathrm{m} / \mathrm{z} 1646.35\left[\mathrm{M}-3 \mathrm{~N}\left(\mathrm{SO}_{3} \mathrm{CF}_{3}\right)_{2}^{-}\right]^{3+}$ (calcd. 
$\mathrm{m} / \mathrm{z}:$ 1646.32), $1164.80\left[\mathrm{M}-4 \mathrm{~N}\left(\mathrm{SO}_{3} \mathrm{CF}_{3}\right)_{2}{ }^{-}\right]^{4+}\left(\right.$ calcd. m/z: 1164.76) and $875.87\left[\mathrm{M}-5 \mathrm{~N}\left(\mathrm{SO}_{3} \mathrm{CF}_{3}\right)_{2}{ }^{-}\right]^{5+}(\mathrm{calcd} . \mathrm{m} / \mathrm{z}$ : 875.82).

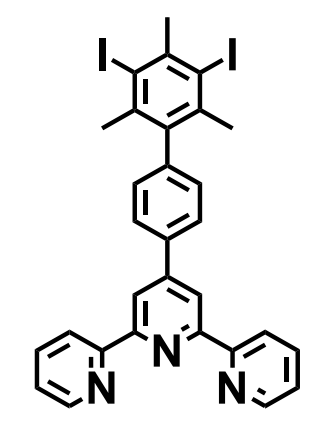

Compound S9:

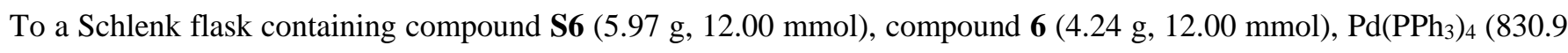
$\mathrm{mg}, 0.72 \mathrm{mmol})$ and $\mathrm{Na}_{2} \mathrm{CO}_{3}(12.72 \mathrm{~g}, 120.00 \mathrm{mmol})$ were added. After the removal of air and back-filled with argon, 80 $\mathrm{mL}$ of toluene, $20 \mathrm{~mL}$ of water and $20 \mathrm{~mL}$ of $\mathrm{EtOH}$ were added. After stirring at $85{ }^{\circ} \mathrm{C}$ for $12 \mathrm{~h}$, the mixture was cooled to $25^{\circ} \mathrm{C}$. The aqueous layer was extracted with $\mathrm{CH}_{2} \mathrm{Cl}_{2}$, and then the combined organic phase was washed with brine and dried with $\mathrm{MgSO}_{4}$. The solvents were then removed under reduced pressure. The residue was purified by column chromatography $\left(\mathrm{Al}_{2} \mathrm{O}_{3}\right)$, eluting with chloroform to afford compound $\mathbf{S 9}$ as white solid $(6.52 \mathrm{~g}, 80 \%) .{ }^{1} \mathrm{H} \mathrm{NMR}(500 \mathrm{MHz}$,

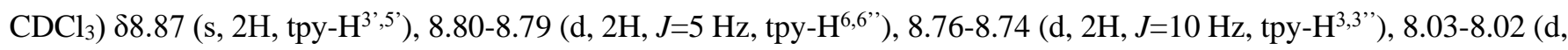

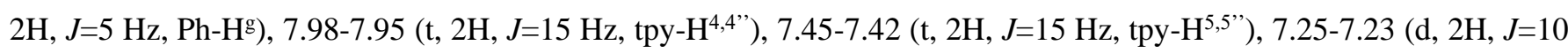
$\mathrm{Hz}, \mathrm{Ph}-\mathrm{H}^{\mathrm{h}}$ ), 3.07 (s, 3H, Me), 2.26 (s, 6H, Me). ${ }^{13} \mathrm{C} \mathrm{NMR}\left(101 \mathrm{MHz}, \mathrm{CDCl}_{3}\right) \delta 156.19,156.01,149.96,149.17,143.42$, $143.14,139.86,139.29,137.53,136.94,129.38,127.79,123.92,121.37,118.98,105.14,39.01,29.35$. ESI-MS (679.34 calcd. For $\left.\mathrm{C}_{30} \mathrm{H}_{23} \mathrm{~N}_{3} \mathrm{I}_{2}\right): \mathrm{m} / \mathrm{z} 679.94[\mathrm{M}+\mathrm{H}]^{+}($calcd. m/z: 680.01$)$.

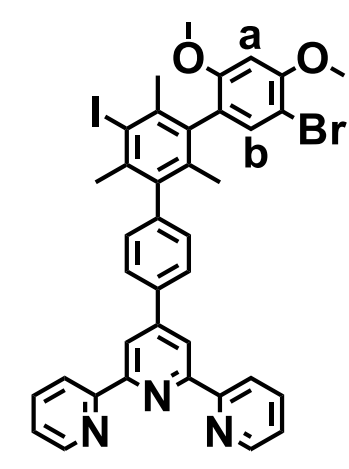

Compound S11: 
Compound S9 (4.08 g, $6.00 \mathrm{mmol}$ ) and (5-bromo-2,4-dimethoxyphenyl)boronic acid (1.57 g, $6.00 \mathrm{mmol})$ was added to a $250 \mathrm{~mL}$ flask, then THF $(90 \mathrm{~mL}) 、 \mathrm{H}_{2} \mathrm{O}(60 \mathrm{~mL})$ and $\mathrm{K}_{2} \mathrm{CO}_{3}(8.29 \mathrm{~g}, 15.00 \mathrm{mmol})$ in $15 \mathrm{~mL}$ of water was added. The system was degassed for $10 \mathrm{~min}$, and $\mathrm{Pd}\left(\mathrm{PPh}_{3}\right)_{2} \mathrm{Cl}_{2}(336.9 \mathrm{mg}, 0.48 \mathrm{mmol})$ as the catalyst was added. The mixture was stirred at $85{ }^{\circ} \mathrm{C}$ under nitrogen for $12 \mathrm{~h}$, after cooled to ambient temperature, then concentrated in vacuo followed by column chromatography $\left(\mathrm{Al}_{2} \mathrm{O}_{3}\right)$, eluting with the mixture of petroleum ether and $\mathrm{CH}_{2} \mathrm{Cl}_{2}$ to pure the product, as white solid: $1.61 \mathrm{~g}, 35 \% .{ }^{1} \mathrm{H}$ NMR (500 MHz, $\left.\mathrm{CDCl}_{3}\right) \delta 8.87\left(\mathrm{~s}, 2 \mathrm{H}, \mathrm{tpy}-\mathrm{H}^{3}, 5^{\prime}\right), 8.80-8.79\left(\mathrm{~d}, 2 \mathrm{H}, J=5 \mathrm{~Hz}\right.$, tpy-H $\left.{ }^{6,6^{\prime}}\right), 8.76-8.75$

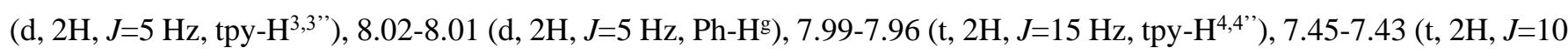
Hz, tpy-H $\left.{ }^{5,5}\right), 7.36-7.34\left(\mathrm{~d}, 2 \mathrm{H}, J=10 \mathrm{~Hz}, \mathrm{Ph}-\mathrm{H}^{\mathrm{h}}\right), 7.22\left(\mathrm{~s}, 1 \mathrm{H}, \mathrm{H}^{\mathrm{b}}\right), 6.61\left(\mathrm{~s}, 1 \mathrm{H}, \mathrm{H}^{\mathrm{a}}\right), 3.99(\mathrm{~s}, 3 \mathrm{H}, \mathrm{OMe}), 3.82(\mathrm{~s}, 3 \mathrm{H}, \mathrm{OMe})$, 2.32 (s, 3H, Me), 2.31 (s, 3H, Me), 1.67 (s, 3H, Me). ${ }^{13} \mathrm{C}$ NMR (101 MHz, $\left.\mathrm{CDCl}_{3}\right) \delta$ 156.77, 156.25, 155.97, 150.16, $149.17,143.18,139.78,139.52,138.88,137.11,136.92,135.18,135.04,134.26,129.79,127.65,124.26,123.88,121.36$, 118.98, 107.85, 101.92. ESI-MS (768.49 calcd. For $\left.\mathrm{C}_{38} \mathrm{H}_{31} \mathrm{BrIN}_{3} \mathrm{O}_{2}\right): \mathrm{m} / \mathrm{z} 770.02[\mathrm{M}+\mathrm{H}]^{+}$(calcd. m/z: 770.07).

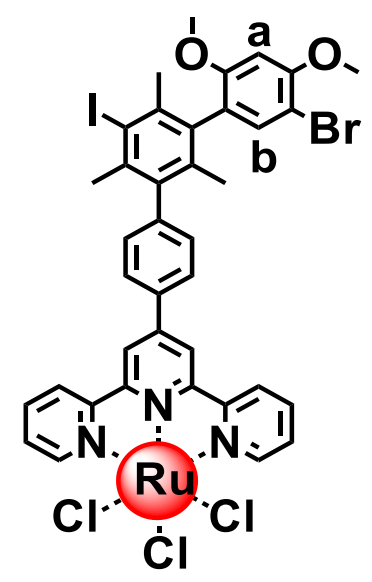

\section{Compound 7:}

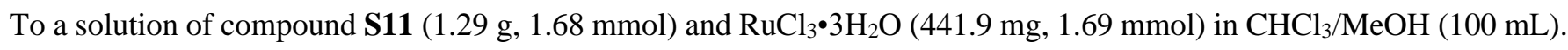
The mixture was stirred at $72{ }^{\circ} \mathrm{C}$ for $24 \mathrm{~h}$. After cooling to ambient temperature, the precipitates were filtered and washed with $\mathrm{MeOH}$ to afford compound 7: $1.46 \mathrm{~g}, 89 \%$. 


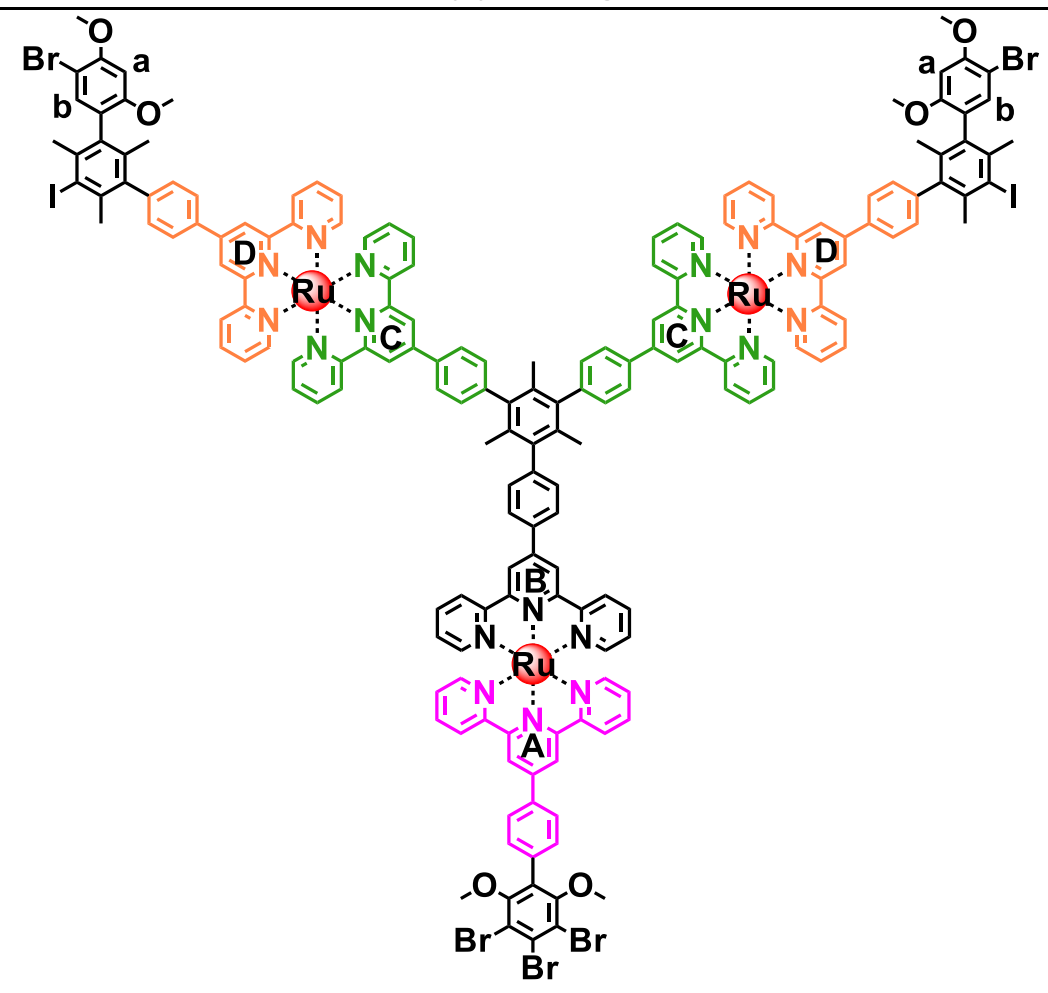

Complex 8:

To a flask containing a mixture of complex $\mathbf{3}(43.0 \mathrm{mg}, 0.02 \mathrm{mmol})$ and compound 7 (68.3 mg, $0.07 \mathrm{mmol}), \mathrm{MeOH}(150$ $\mathrm{mL}), \mathrm{CHCl}_{3}(150 \mathrm{~mL})$, and drops of N-ethylmorpholine were added. The mixture was stirred at $80{ }^{\circ} \mathrm{C}$ for $20 \mathrm{~h}$. After cooling to ambient temperature, the solvent was evaporated in vacuo and the residue was subjected to column chromatography $\left(\mathrm{Al}_{2} \mathrm{O}_{3}, \mathrm{CH}_{2} \mathrm{Cl}_{2} / \mathrm{MeOH}\right)$ to afford complex 8 as red solid: $66.5 \mathrm{mg}, 88 \% .{ }^{1} \mathrm{H}$ NMR $(500 \mathrm{MHz}, \mathrm{MeOD}) \delta$

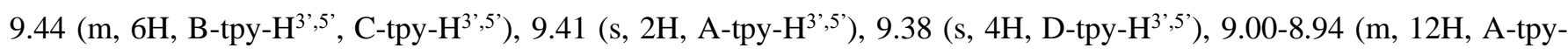

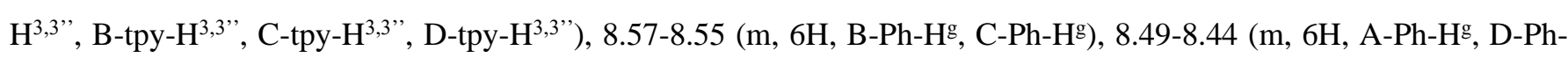

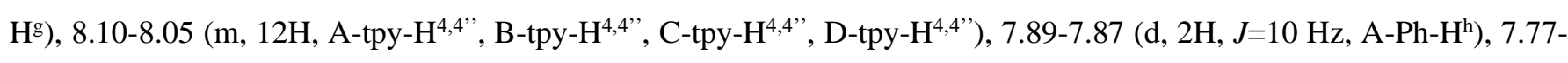

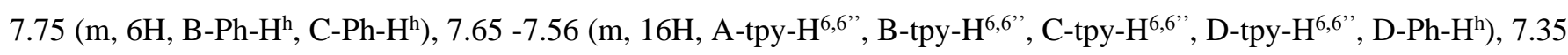

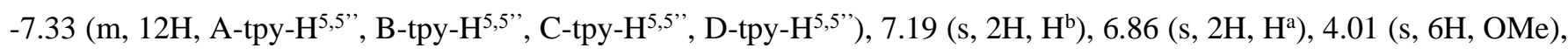
3.87 (s, 6H, OMe), 3.55 (s, 6H, OMe), 2.39 (s, 6H, Me), 2.30 (s, 6H, Me), 2.06 (s, 9H, Me), 1.74 (s, 6H, Me). ${ }^{13} \mathrm{C}$ NMR (101 MHz, MeOD) $\delta 158.45,156.97,156.52,155.92,155.68,155.63,155.61,152.00,148.71,148.66,148.59,148.24$, $144.74,144.37,139.80,139.55,139.15,138.09,137.99,136.64,135.52,134.57,133.55,132.78,131.38,130.68,130.40$, 130.37, 128.17, 127.96, 127.60, 127.41, 124.70, 123.78, 121.47, 117.60, 106.69, 101.39, 96.85, 59.90, 56.30, 56.08, 55.86, $55.57, \quad 55.05, \quad 28.04, \quad 27.59, \quad 18.59, \quad 17.99, \quad 16.26, \quad 16.07,15.88, \quad 15.69$. ESI-MS (3777.37 calcd. For $\left.\mathrm{C}_{177} \mathrm{H}_{133} \mathrm{Br}_{5} \mathrm{I}_{2} \mathrm{Cl}_{6} \mathrm{~N}_{18} \mathrm{O}_{6} \mathrm{Ru}_{3}\right): \mathrm{m} / \mathrm{z} 1223.54\left[\mathrm{M}-3 \mathrm{Cl}^{-}\right]^{3+}$ (calcd. m/z: 1223.69), 908.93 [M-4Cl- $]^{4+}$ (calcd. m/z: 909.03$)$ and $720.15\left[\mathrm{M}-5 \mathrm{Cl}^{-}\right]^{5+}$ (calcd. m/z: 720.03). 


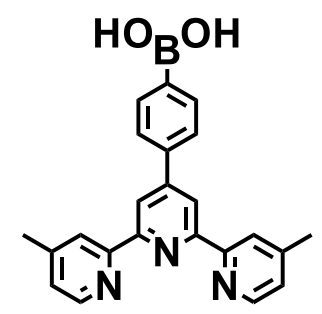

Compound 9:

To a solution of (4-formylphenyl)boronic acid (1.31 g, $8.74 \mathrm{mmol})$ and 1-(4-methylpyridin-2-yl)ethan-1-one (2.60 g, 19.20 mmol) in $\mathrm{EtOH}(30 \mathrm{~mL}), \mathrm{NaOH}$ powder $(2.10 \mathrm{~g}, 52.4 \mathrm{mmol})$ was added. After stirring at $25{ }^{\circ} \mathrm{C}$ for $10 \mathrm{~h}$, aqueous ammonium hydroxide $(20 \mathrm{~mL}, 30 \%)$ was added, and the mixture was kept refluxed for another $20 \mathrm{~h}$. After cooling to room temperature, the volatiles were removed. $50 \mathrm{~mL}$ water was added to the residue, and the mixture was sonicated for $2 \mathrm{~h}$. After centrifuge at $5000 \mathrm{rpm}$ for $5 \mathrm{mins}$, the precipitate was collected and washed with $\mathrm{CHCl}_{3}$ to give compound 9 as white

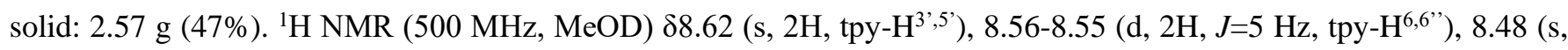

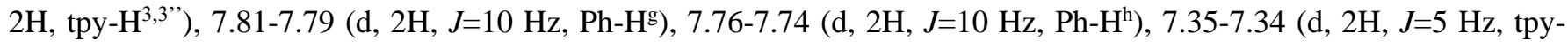
$\left.\mathrm{H}^{5,5 ”}\right), 2.55$ (s, 6H, Me). ${ }^{13} \mathrm{C}$ NMR (126 MHz, MeOD) $\delta 156.01,155.69,151.64,149.18,148.34,141.38,134.25,133.79$, 124.80, 124.62, 122.45, 118.32, 19.94. ESI-MS (381.24 calcd. For $\left.\mathrm{C}_{23} \mathrm{H}_{20} \mathrm{BN}_{3} \mathrm{O}_{2}\right): \mathrm{m} / \mathrm{z} 382.17[\mathrm{M}+\mathrm{H}]^{+}$(calcd. m/z: 382.17$)$.

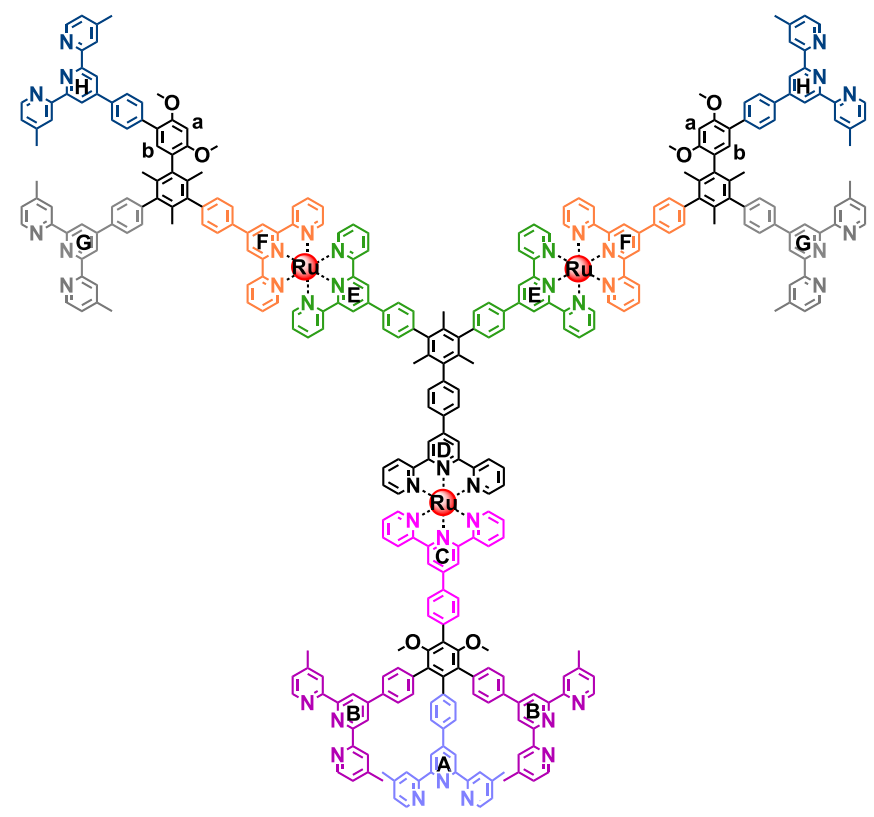

Ligand LB:

Complex $8(50.0 \mathrm{mg}, 0.01 \mathrm{mmol})$ and compound $9(282.5 \mathrm{mg}, 0.74 \mathrm{mmol})$ was added to a $100 \mathrm{~mL}$ flask, then acetonitrile (45 mL), methanol $(15 \mathrm{~mL})$ and $\mathrm{K}_{2} \mathrm{CO}_{3}(64.0 \mathrm{mg}, 0.46 \mathrm{mmol})$ in $0.3 \mathrm{~mL}$ of water was added. The system was degassed 
for $10 \mathrm{~min}$, and $\mathrm{Pd}\left(\mathrm{PPh}_{3}\right)_{4}(32.0 \mathrm{mg}, 0.03 \mathrm{mmol})$ as the catalyst was added. The mixture was stirred at $85^{\circ} \mathrm{C}$ under nitrogen for $7 \mathrm{~d}$, after cooled to ambient temperature, then concentrated in vacuo followed by column chromatography $\left(\mathrm{Al}_{2} \mathrm{O}_{3}\right)$, eluting with the mixture of petroleum ether and $\mathrm{CH}_{2} \mathrm{Cl}_{2}$ to pure the product. The complex was counterion exchanged with $\mathrm{LiN}\left(\mathrm{SO}_{2} \mathrm{CF}_{3}\right)_{2}$ to give ligand $\mathbf{L B}$, as a red precipitate: $34.9 \mathrm{mg}, 38 \% .{ }^{1} \mathrm{H} \mathrm{NMR}(500 \mathrm{MHz}, \mathrm{DMSO}) \delta 9.64-9.59(\mathrm{~m}, 10 \mathrm{H}$,

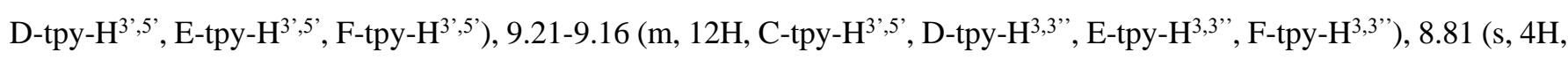

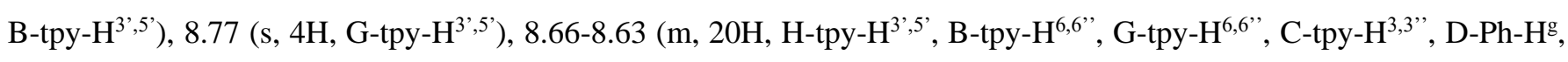

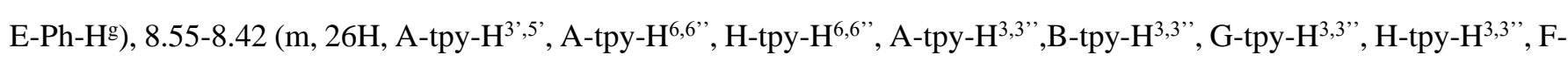

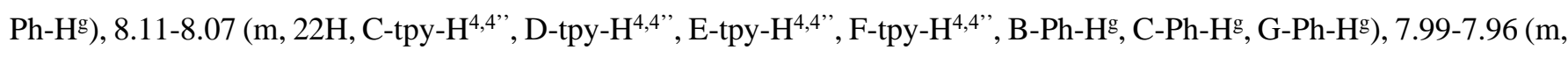
6H, A-Ph-H $\left.{ }^{\mathrm{g}}, \mathrm{H}-\mathrm{Ph}-\mathrm{H}^{\mathrm{g}}\right)$, 7.85-7.70 (m, 20H, B-Ph-H $\left.{ }^{\mathrm{h}}, \mathrm{C}-\mathrm{Ph}-\mathrm{H}^{\mathrm{h}}, \mathrm{D}-\mathrm{Ph}-\mathrm{H}^{\mathrm{h}}, \mathrm{E}-\mathrm{Ph}-\mathrm{H}^{\mathrm{h}}, \mathrm{F}-\mathrm{Ph}-\mathrm{H}^{\mathrm{h}}, \mathrm{G}-\mathrm{Ph}-\mathrm{H}^{\mathrm{h}}\right), 7.62-7.50(\mathrm{~m}$,

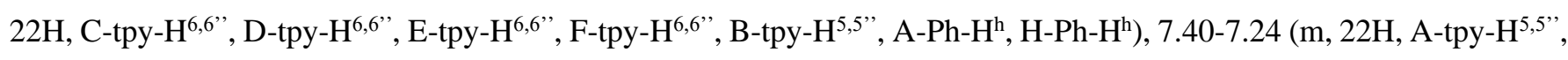

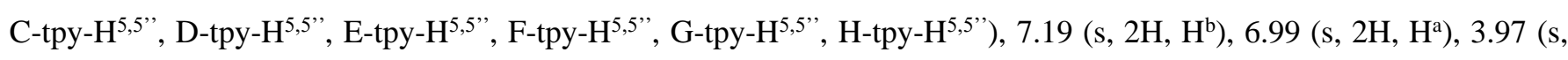
6H, OMe), 3.94 (s, 6H, OMe), 3.21 (s, 6H, OMe). ESI-MS (6947.08 calcd. For $\mathrm{C}_{350} \mathrm{H}_{259} \mathrm{~F}_{36} \mathrm{~N}_{45} \mathrm{O}_{30} \mathrm{Ru}_{3} \mathrm{~S}_{12}$ ): m/z 1456.40 $\left[\mathrm{M}-4 \mathrm{~N}\left(\mathrm{SO}_{3} \mathrm{CF}_{3}\right)_{2}^{-}\right]^{4+}\left(\right.$ calcd. m/z: 1456.42), $1109.17\left[\mathrm{M}-5 \mathrm{~N}\left(\mathrm{SO}_{3} \mathrm{CF}_{3}\right)_{2}^{-}\right]^{5+}($ calcd. m/z: 1109.15$)$ and $877.69[\mathrm{M}-$ $\left.6 \mathrm{~N}\left(\mathrm{SO}_{3} \mathrm{CF}_{3}\right)_{2}^{-}\right]^{6+}($ calcd. m/z: 877.80).

\section{Ligand LH:}

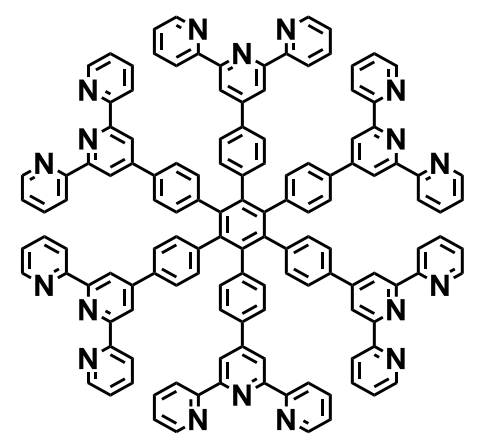

Compound $\mathbf{S 1 4}(1.00 \mathrm{~g}, 1.81 \mathrm{mmol})$ and compound $\mathbf{6}(5.00 \mathrm{~g}, 14.16 \mathrm{mmol})$ was added to a $250 \mathrm{~mL}$ flask, then $\mathrm{K}_{2} \mathrm{CO}_{3}$ $(4.50 \mathrm{~g}, 32.56 \mathrm{mmol}), 60 \mathrm{~mL}$ of toluene, $60 \mathrm{~mL}$ of water and $20 \mathrm{~mL}$ of t-butanol were added. After the removal of air and back-filled with argon, $\mathrm{Pd}\left(\mathrm{PPh}_{3}\right)_{2} \mathrm{Cl}_{2}(281.0 \mathrm{mg}, 0.40 \mathrm{mmol})$ were added. The mixture was stirred at $85^{\circ} \mathrm{C}$ under nitrogen for $6 \mathrm{~d}$, after cooled to ambient temperature, then concentrated in vacuo followed by column chromatography $\left(\mathrm{Al}_{2} \mathrm{O}_{3}\right)$, eluting with the mixture of petroleum ether and $\mathrm{CH}_{2} \mathrm{Cl}_{2}$ to pure the product, as white solid: $2.44 \mathrm{~g}, 70 \%$. ${ }^{1} \mathrm{H} \mathrm{NMR}(500$

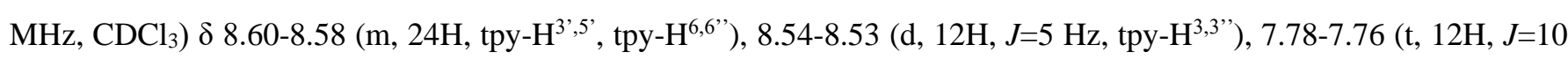

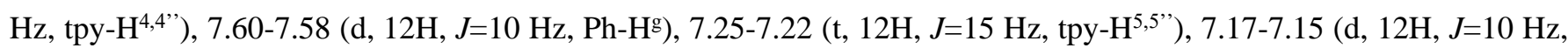
$\left.\mathrm{Ph}-\mathrm{H}^{\mathrm{h}}\right) .{ }^{13} \mathrm{C} \mathrm{NMR}\left(101 \mathrm{MHz}, \mathrm{CDCl}_{3}\right) \delta 156.36,155.59,149.62,148.95,141.27,140.28,136.60,135.26,131.89,126.03$, 123.46, 121.18, 118.75. MALDI-TOF-MS For $\mathrm{C}_{132} \mathrm{H}_{84} \mathrm{~N}_{18}: \mathrm{m} / \mathrm{z} 1922.88[\mathrm{M}+\mathrm{H}]^{+}$(calcd. m/z: 1922.72). 
5. ${ }^{1} \mathrm{H}$ NMR, COSY NMR, NOESY NMR and ${ }^{13} \mathrm{C}$ NMR spectra.
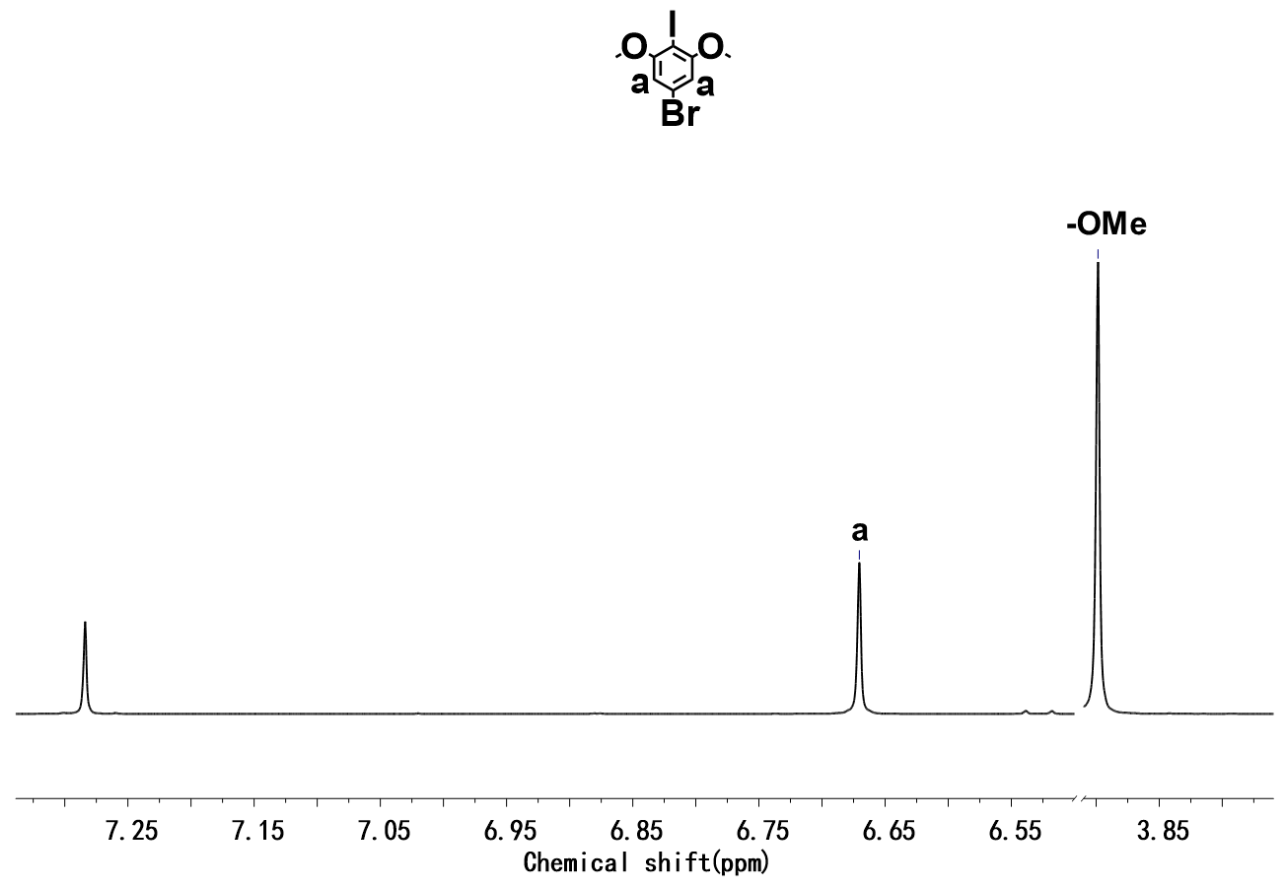

Figure S1: ${ }^{1} \mathrm{H}$ NMR spectrum $(500 \mathrm{MHz})$ of compound $\mathbf{S 3}$ in $\mathrm{CDCl}_{3}$.
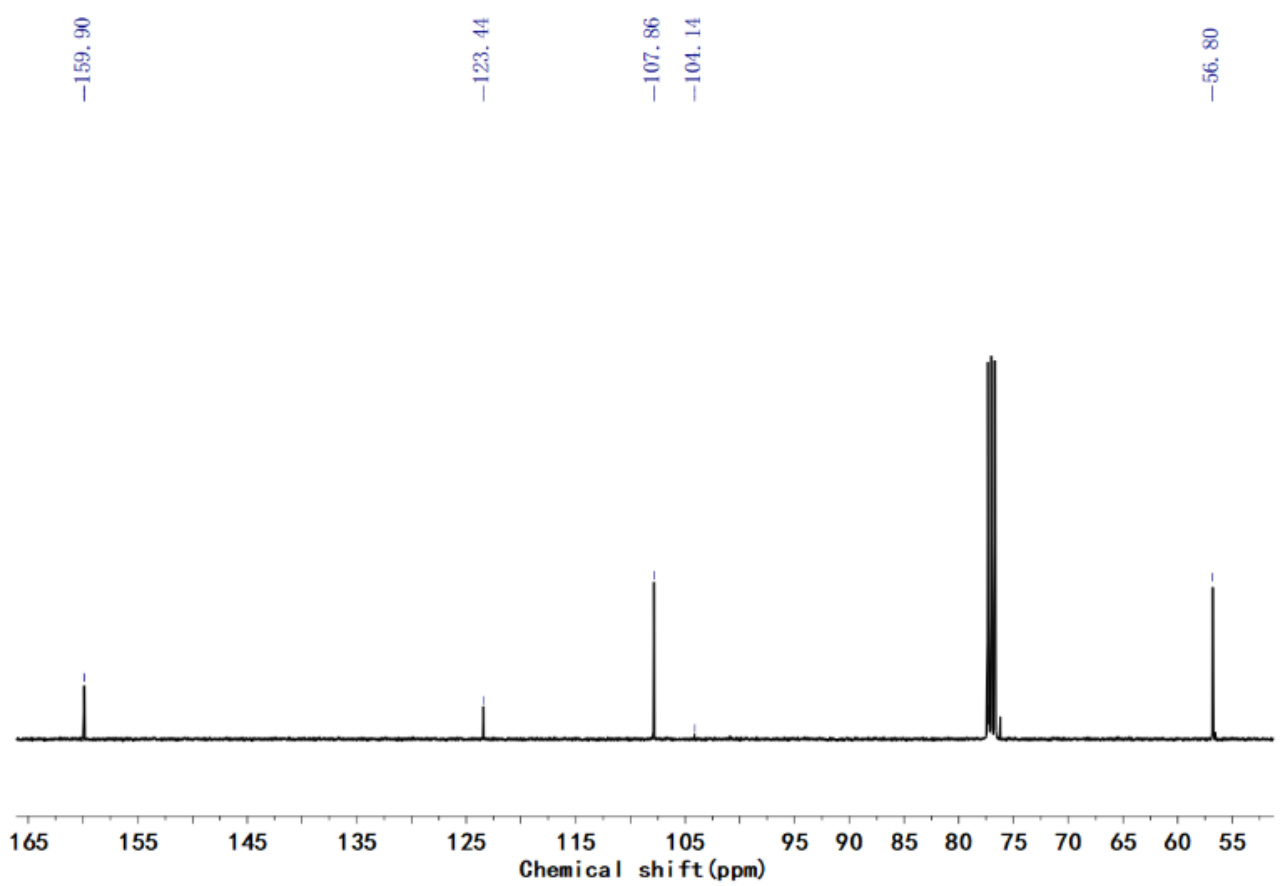

Figure S2: ${ }^{13} \mathrm{C}$ NMR spectrum (101 $\mathbf{M H z}$ ) of compound $\mathbf{S 3}$ in $\mathrm{CDCl}_{3}$. 


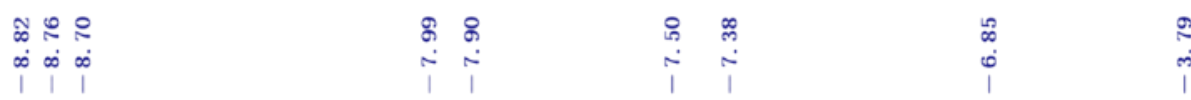

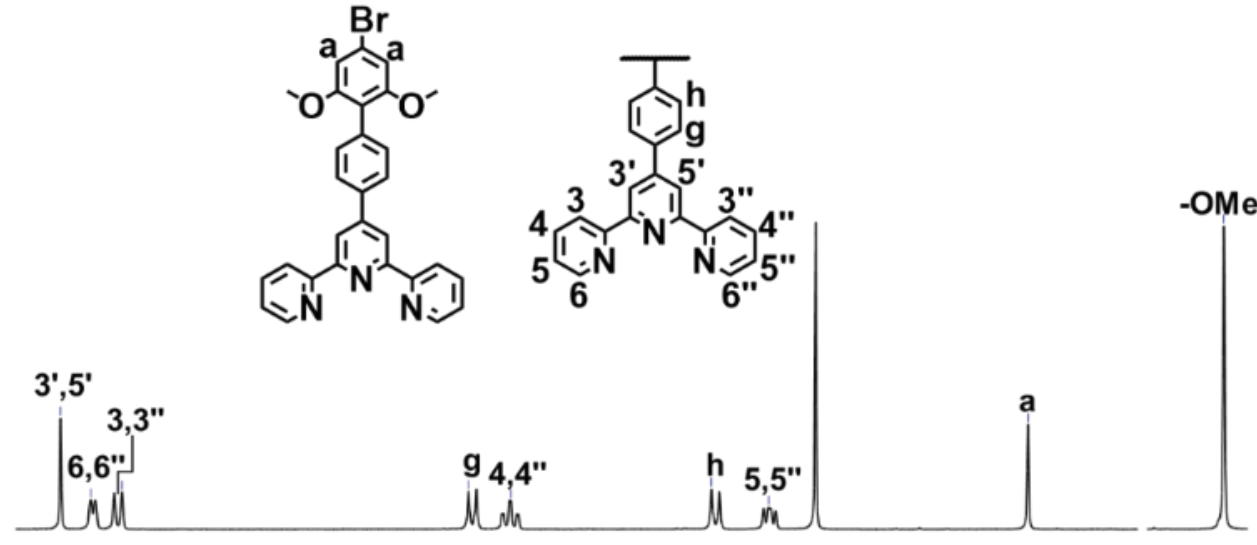

$\begin{array}{llllllllllll}8.8 & 8.6 & 8.4 & 8.2 & 8.0 & 7.8 & 7.6 & 7.4 & 7.2 & 7.0 & 6.8 & 3.9\end{array}$

Figure S3: ${ }^{1} \mathrm{H}$ NMR spectrum $(500 \mathrm{MHz})$ of compound $\mathbf{S 4}$ in $\mathrm{CDCl}_{3}$.

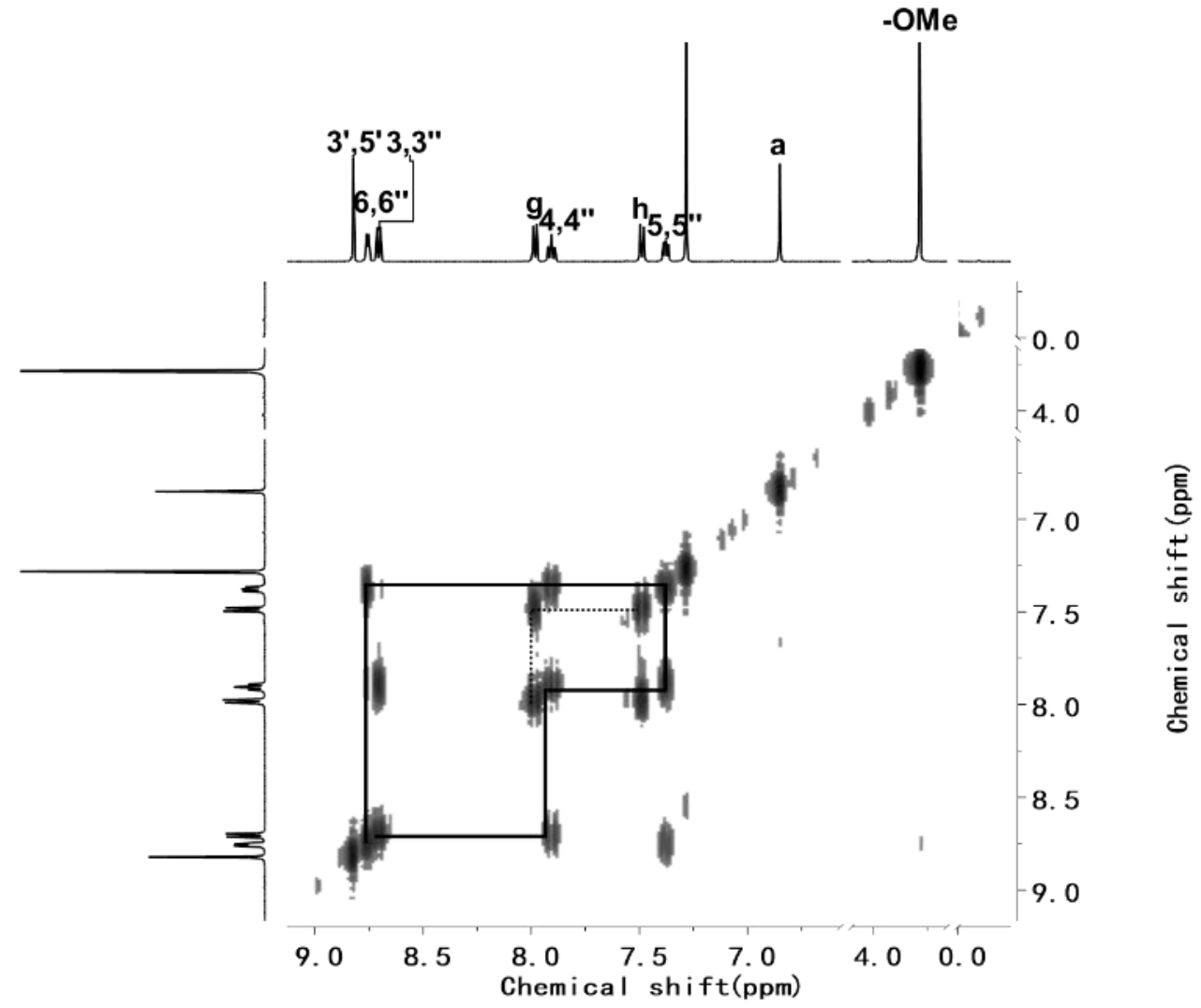

Figure S4: 2D COSY spectrum (500 MHz) of compound $\mathbf{S} 4$ in $\mathrm{CDCl}_{3}$. 


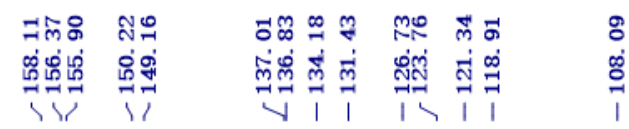

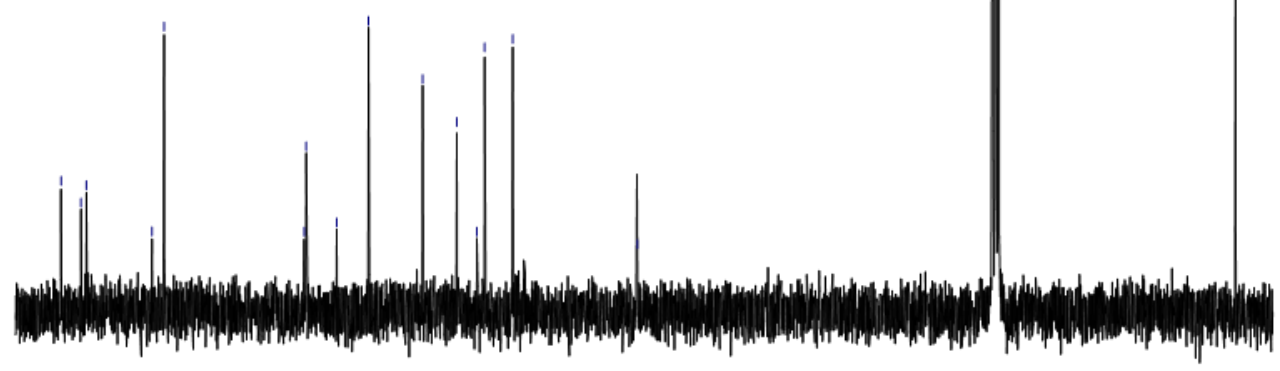

$\begin{array}{lllllllllllllll}155 & 145 & 135 & 125 & \begin{array}{c}115 \\ \text { Chemical }\end{array} & 105 & 95 & 90 & 85 & 80 & 75 & 70 & 65 & 60 & 55\end{array}$

Figure S5: ${ }^{13} \mathrm{C}$ NMR spectrum $(126 \mathrm{MHz})$ of compound $\mathbf{S 4}$ in $\mathrm{CDCl}_{3}$.

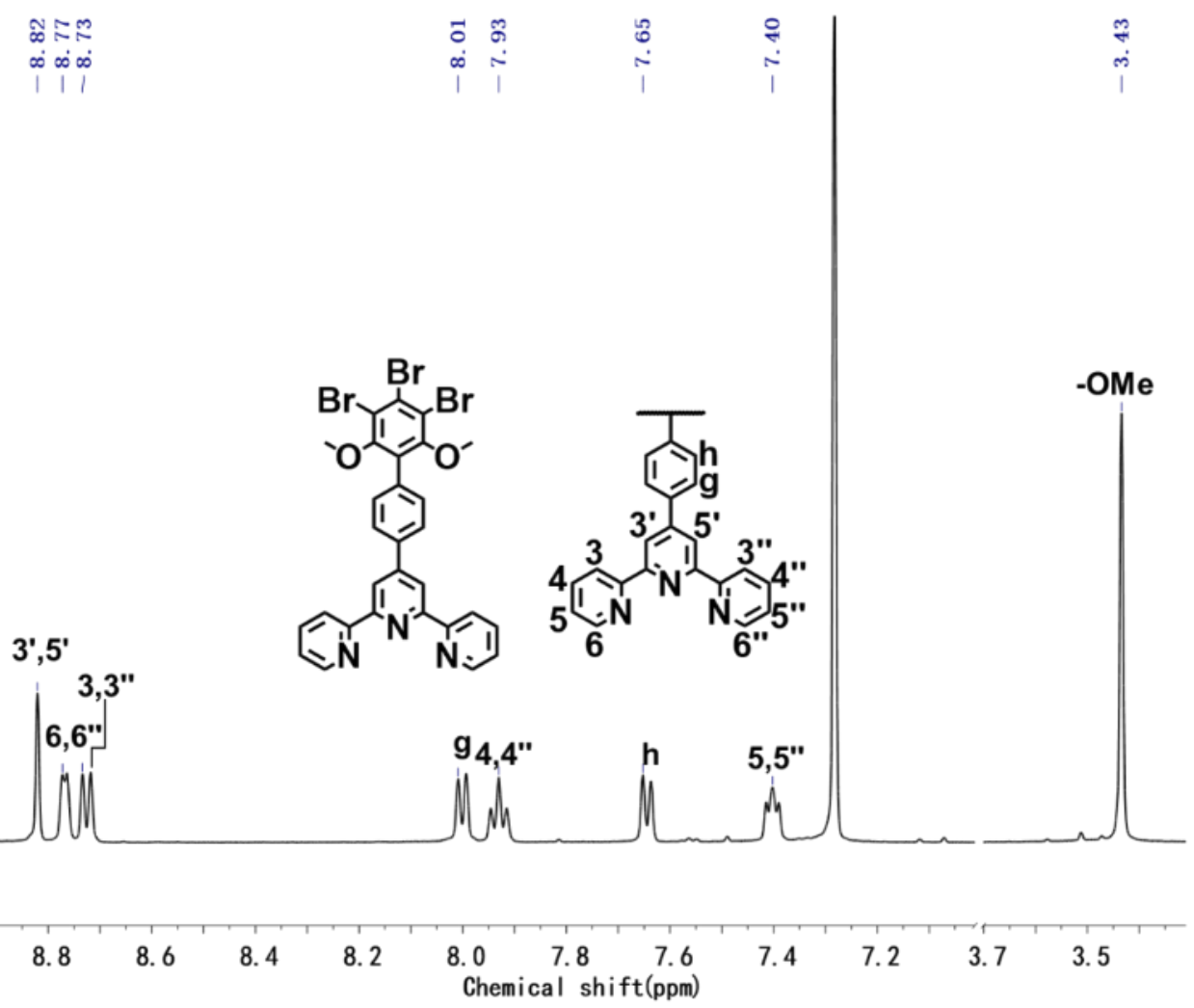

Figure S6: ${ }^{1} \mathrm{H}$ NMR spectrum $(500 \mathrm{MHz})$ of compound $\mathbf{S 5}$ in $\mathrm{CDCl}_{3}$. 


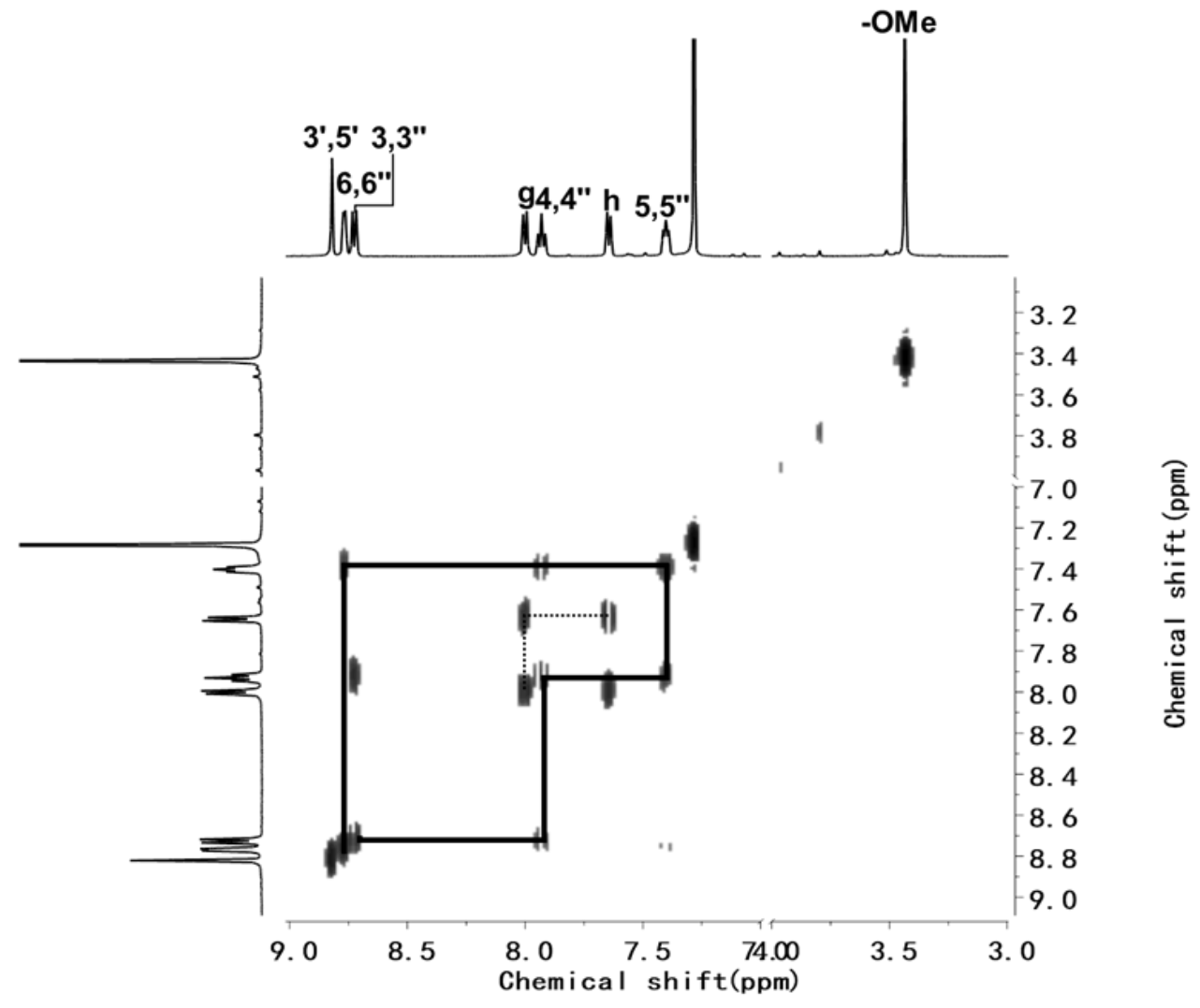

Figure S7: 2D COSY spectrum (500 MHz) of compound $\mathbf{S 5}$ in $\mathrm{CDCl}_{3}$.

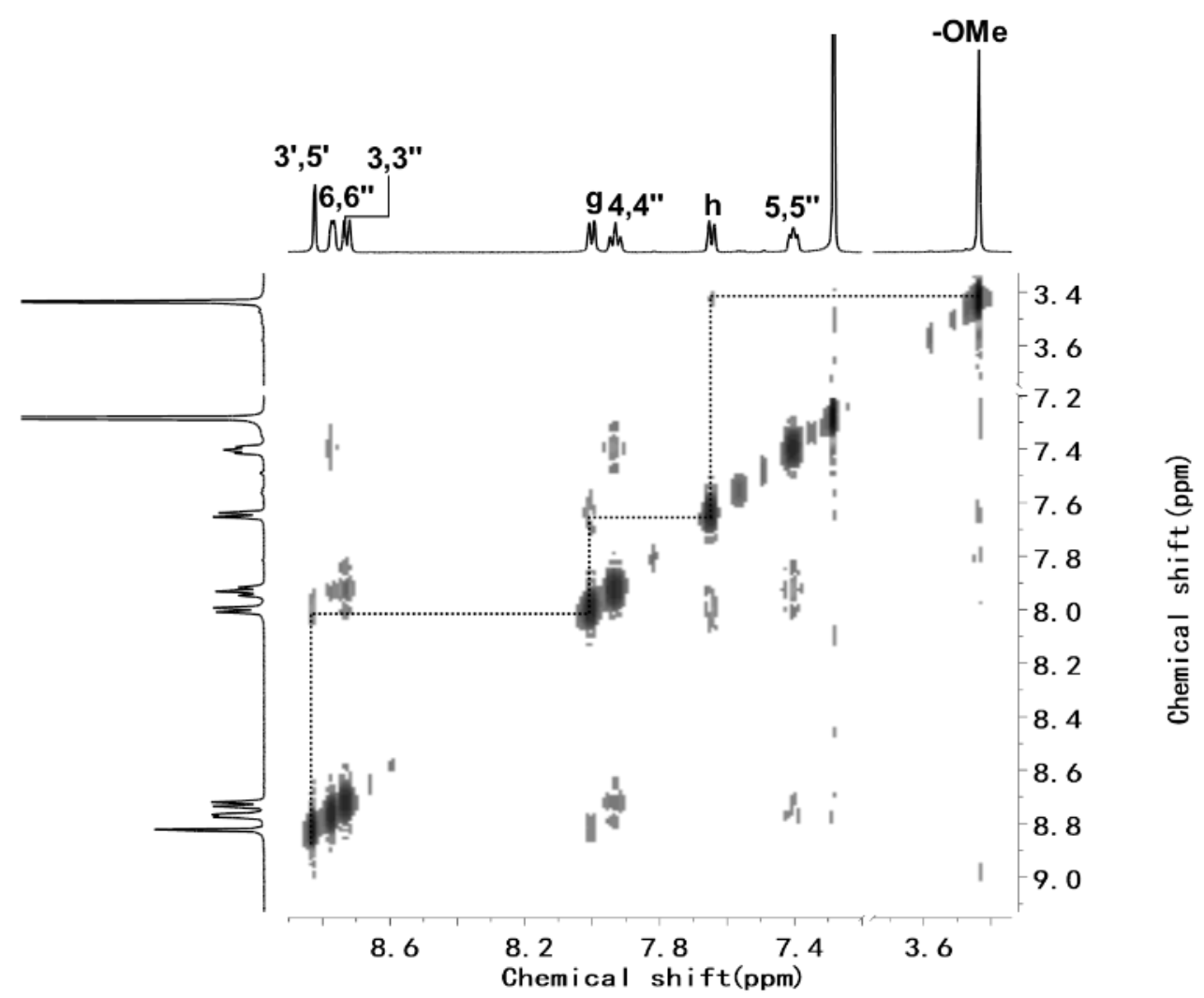

Figure S8: 2D NOESY spectrum (500 MHz) of compound $\mathbf{S 5}$ in $\mathrm{CDCl}_{3}$. 

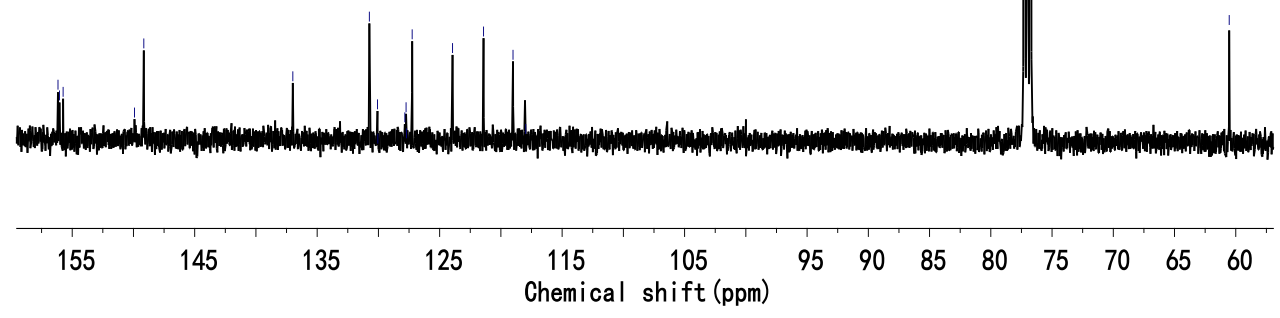

Figure S9: ${ }^{13} \mathrm{C}$ NMR spectrum (126 MHz) of compound $\mathbf{S 5}$ in $\mathrm{CDCl}_{3}$.

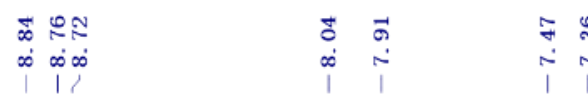

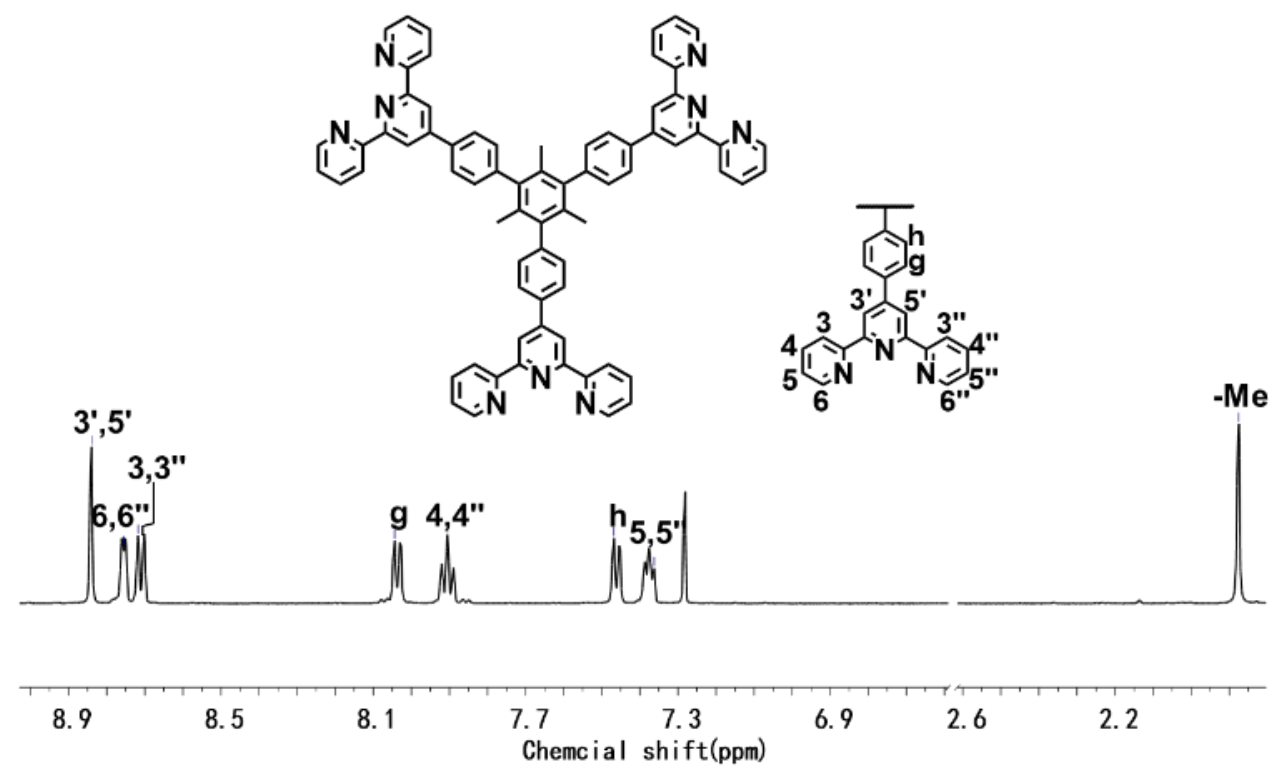

Figure S10: ${ }^{1} \mathrm{H}$ NMR spectrum $(500 \mathrm{MHz})$ of compound 2 in $\mathrm{CDCl}_{3}$. 


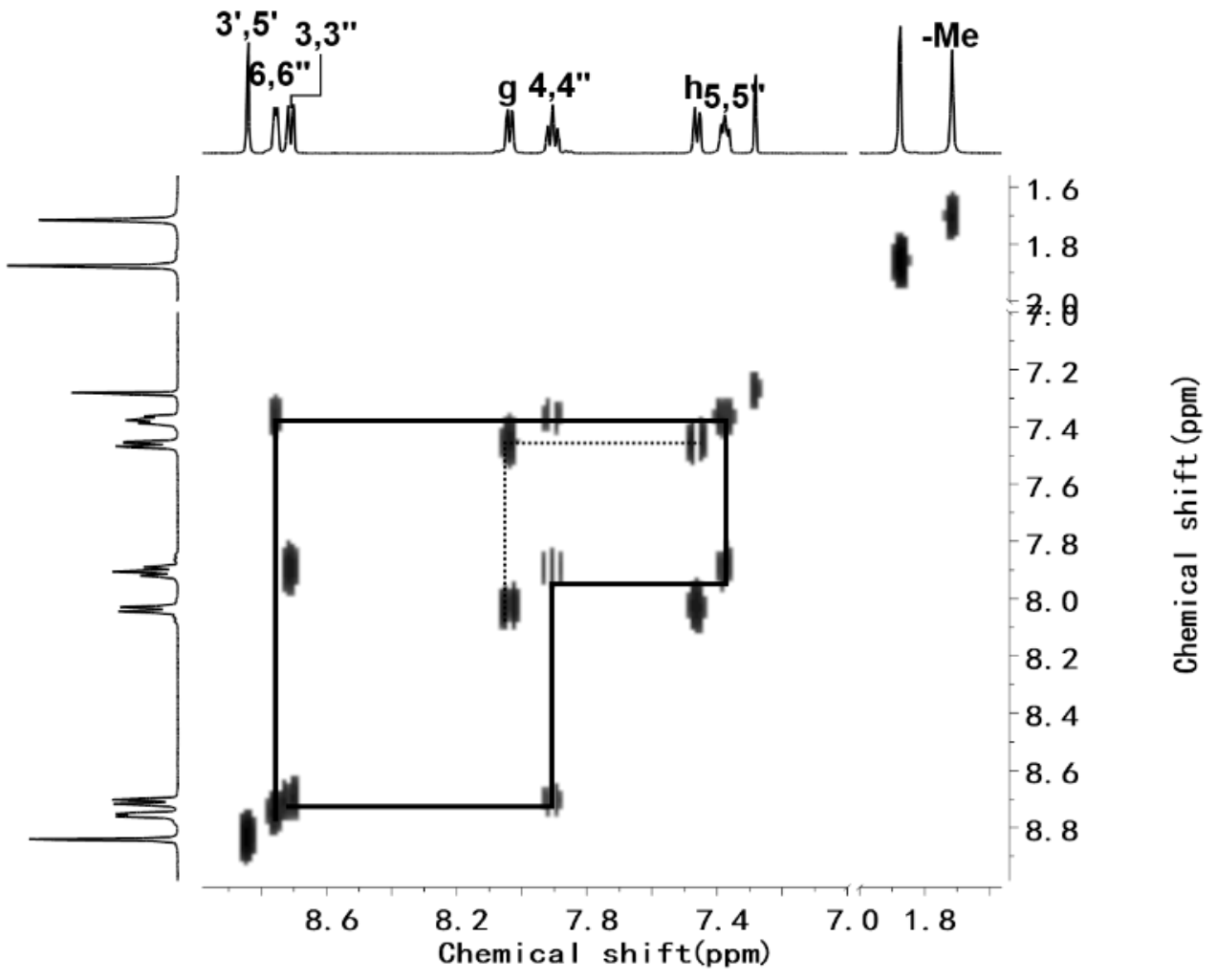

Figure S11: 2D COSY spectrum (500 MHz) of compound 2 in $\mathrm{CDCl}_{3}$.

$$
\begin{aligned}
& \text { लำ 느 }
\end{aligned}
$$

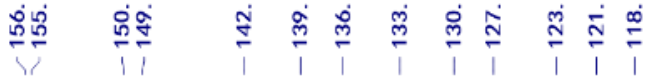

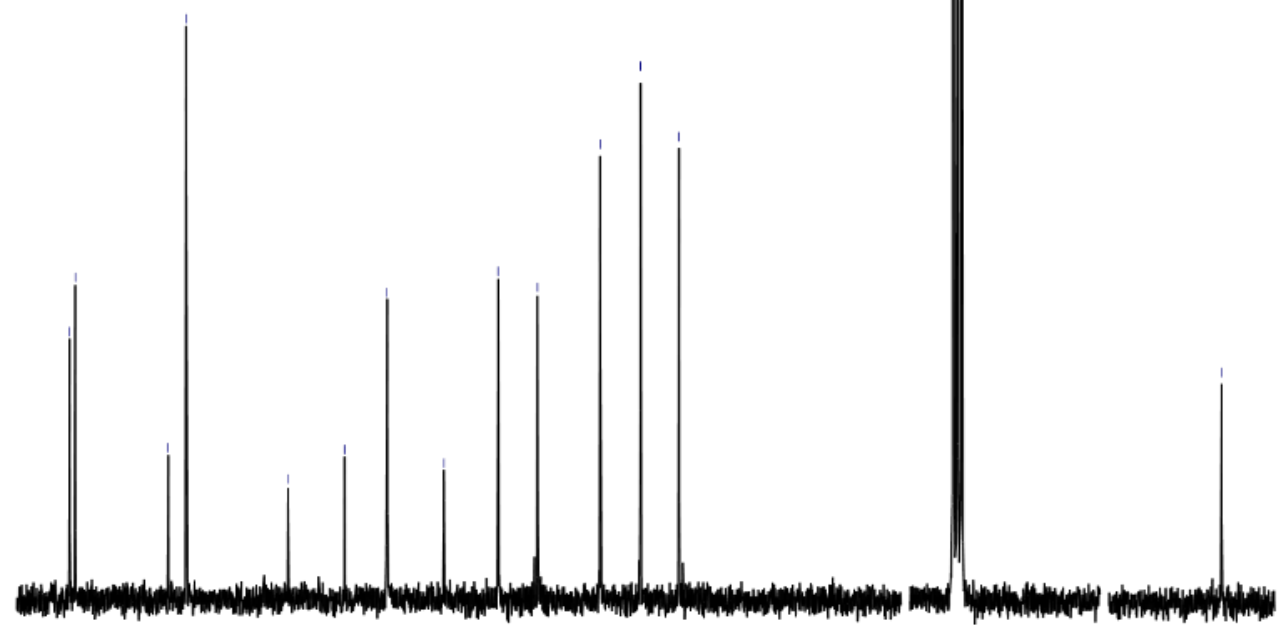

$\begin{array}{llllllllllllll}155 & 150 & 145 & 140 & 135 & 130 & 125 & 120 & 115 & 110 & 75 & 70 & 25 & 20 \\ \text { Chemical shift(ppm) }\end{array}$

Figure S12: ${ }^{13} \mathrm{C}$ NMR spectrum (126 MHz) of compound 2 in $\mathrm{CDCl}_{3}$. 


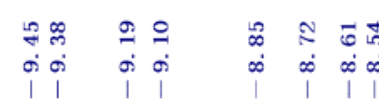

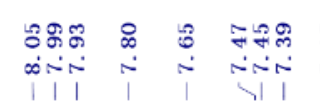

$\stackrel{9}{i}$

- $3^{\prime}, 5^{\prime}=\mathrm{A}$

A $3,3^{\prime \prime}$

$-4,4 "=B$

- 5,5" C

- 6,6"
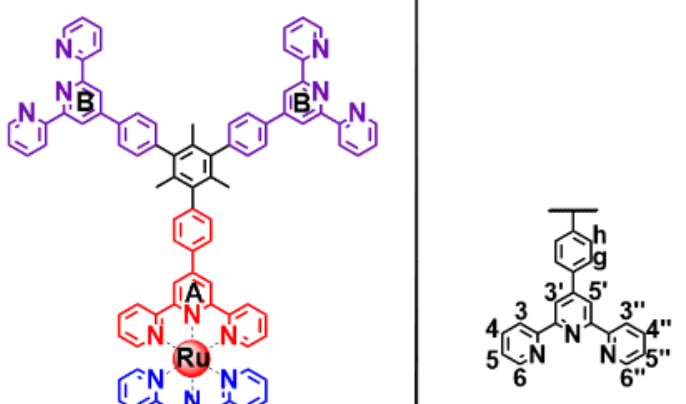

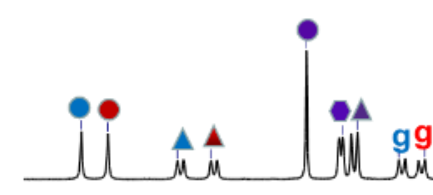

9.5

9.1

8.7

8. 3

7.9

Chemical shift(ppm)

3. 7

Figure S13: ${ }^{1} \mathrm{H}$ NMR spectrum $(500 \mathrm{MHz})$ of complex 3 in $\mathrm{CDCl}_{3}$.

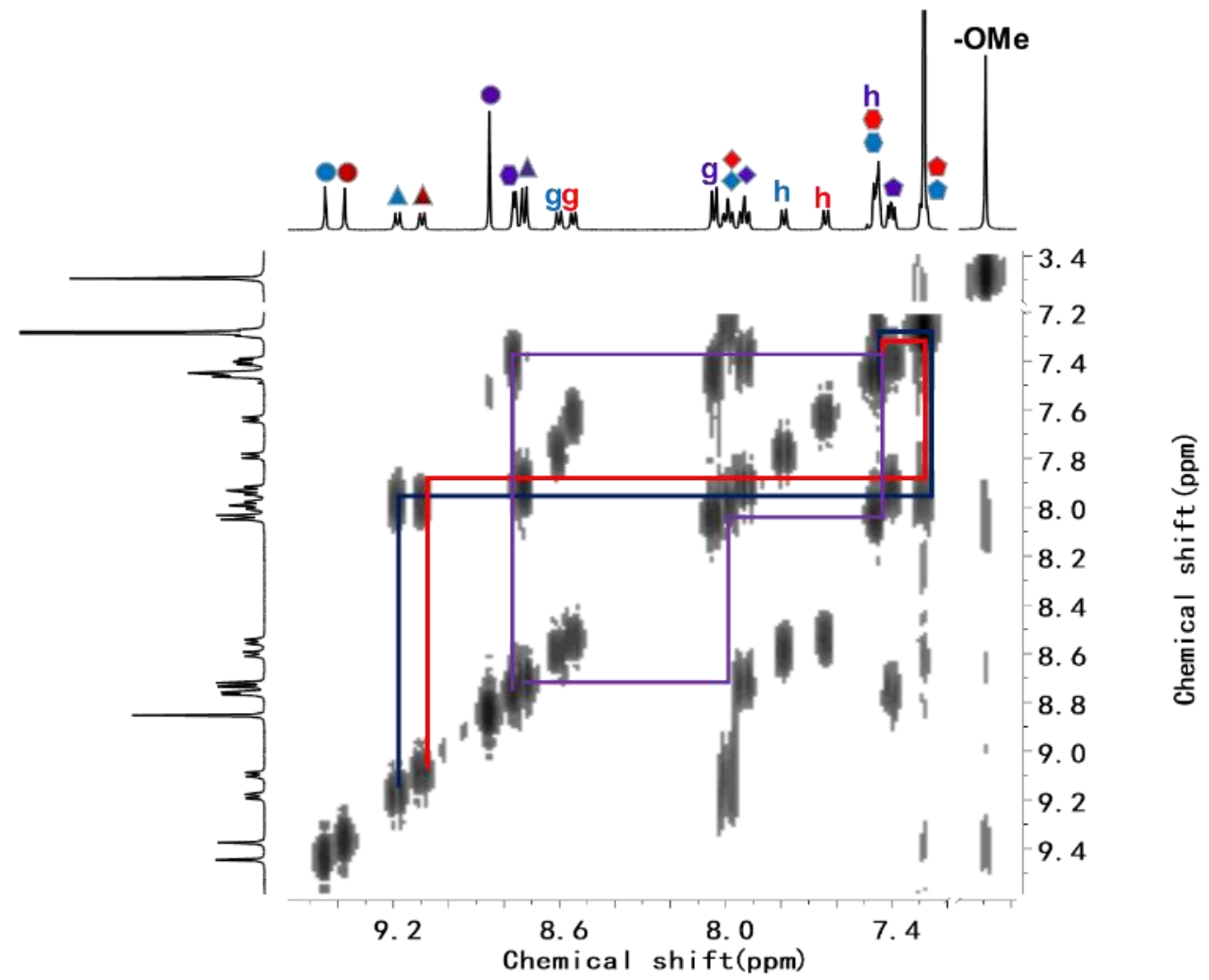

Figure S14: 2D COSY spectrum (500 MHz) of complex 3 in $\mathrm{CDCl}_{3}$. 


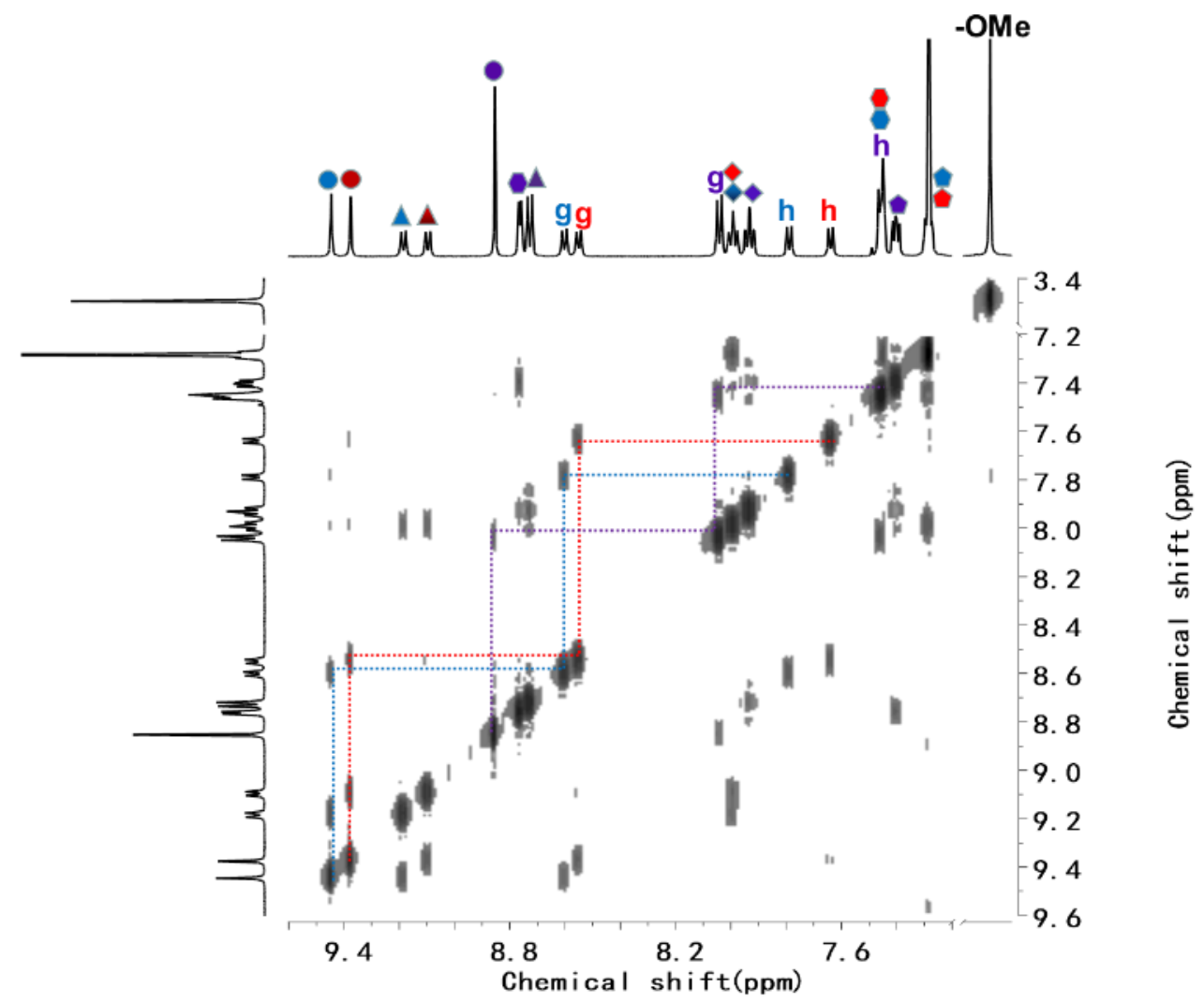

Figure S15: 2D NOESY spectrum (500 MHz) of complex 3 in $\mathrm{CDCl}_{3}$.
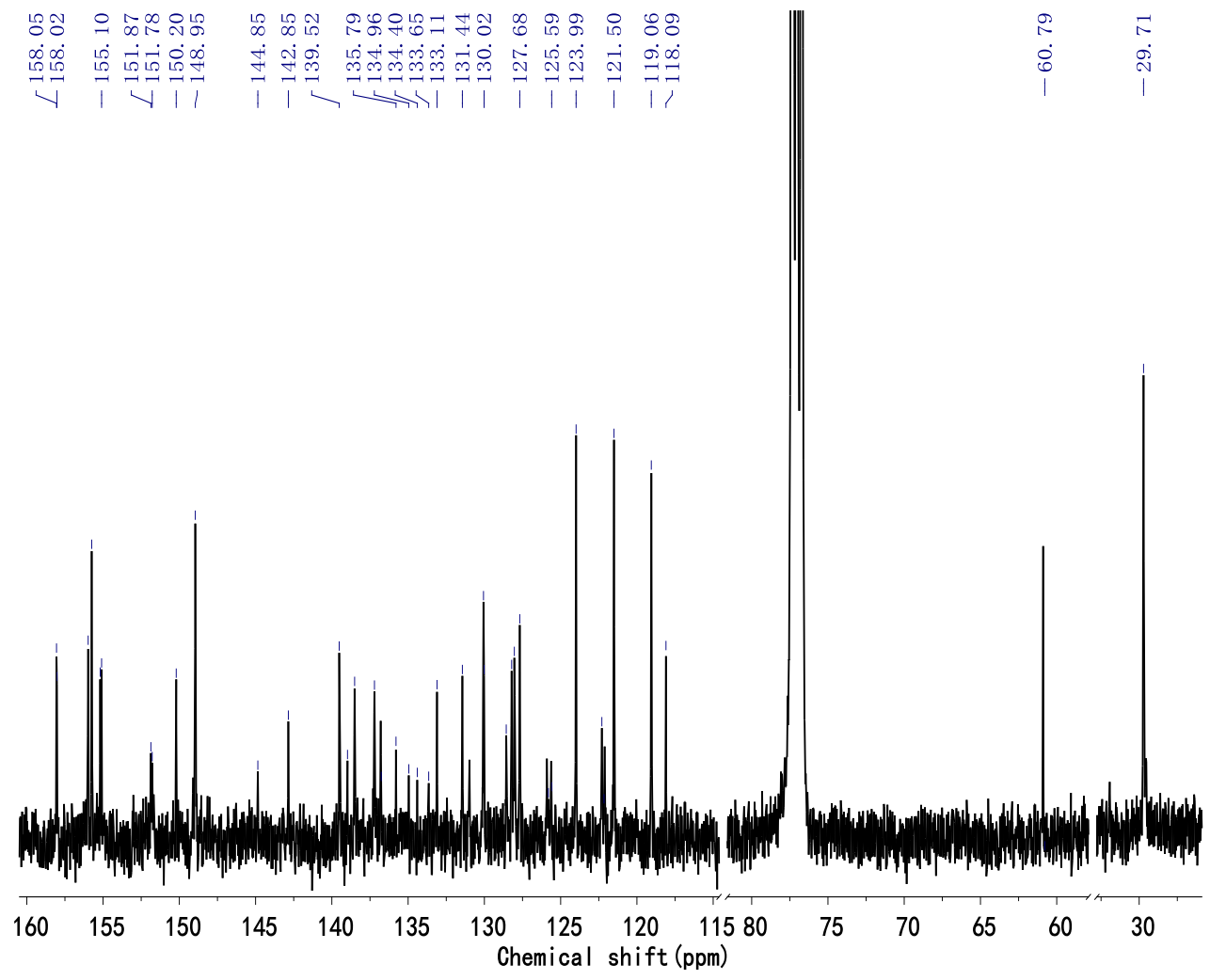

Figure S16: ${ }^{13} \mathrm{C}$ NMR spectrum (126 MHz) of complex 3 in $\mathrm{CDCl}_{3}$. 
कก ก

$\infty \infty \infty$

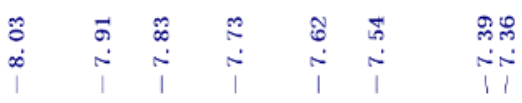
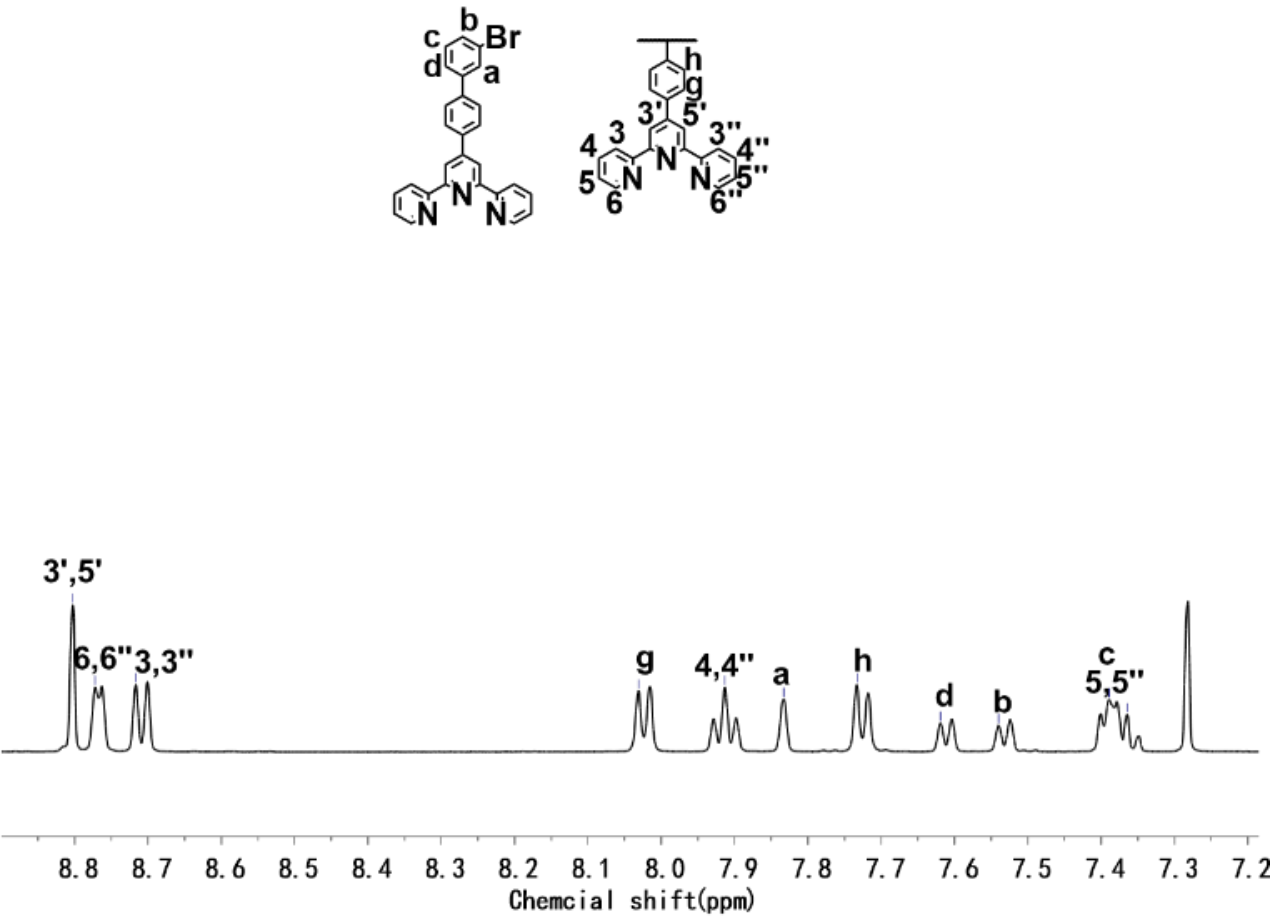

Figure S17: ${ }^{1} \mathrm{H}$ NMR spectrum $(500 \mathrm{MHz})$ of compound $\mathbf{S 8}$ in $\mathrm{CDCl}_{3}$.

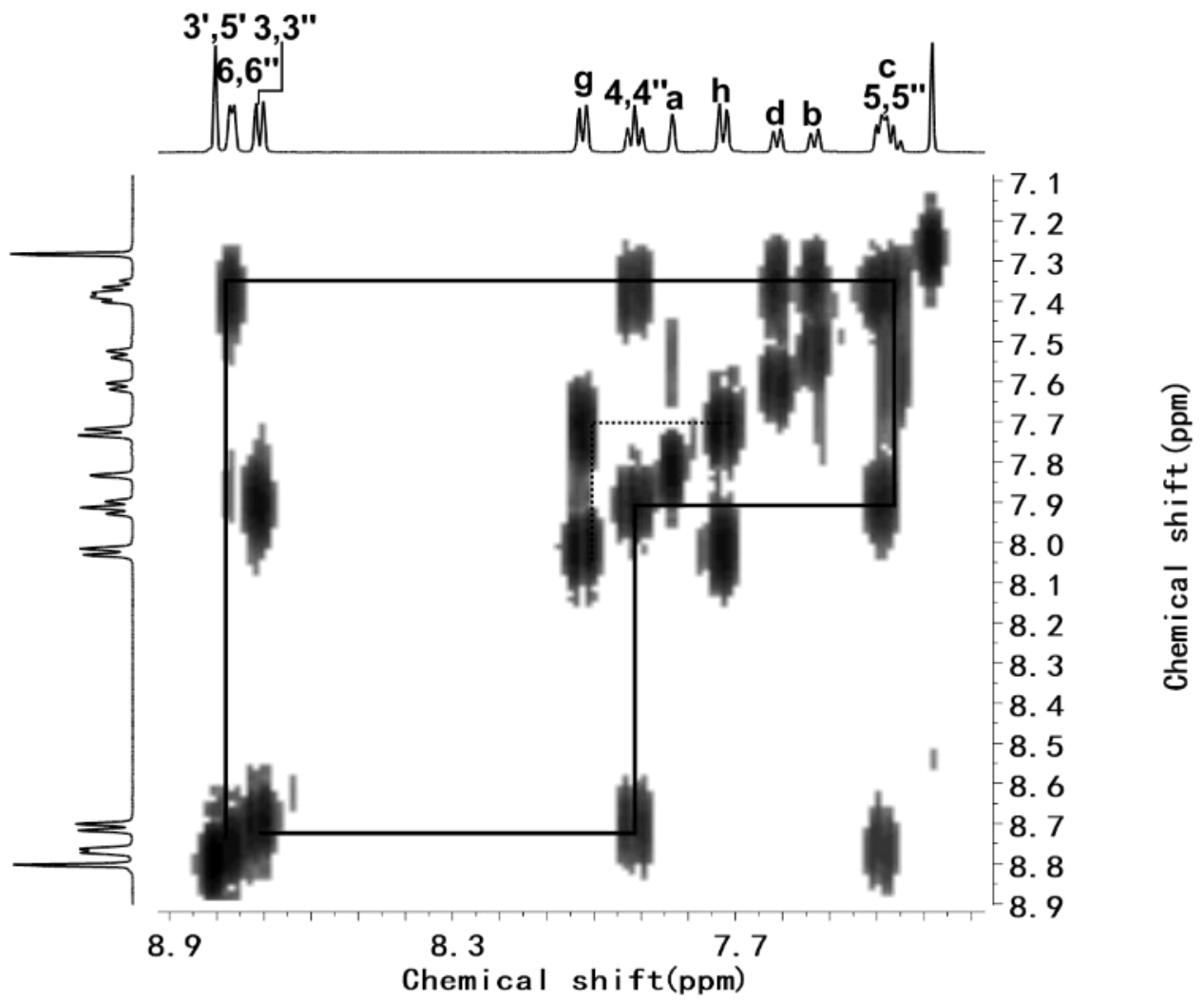

Figure S18: 2D COSY spectrum (500 MHz) of compound $\mathbf{S 8}$ in $\mathrm{CDCl}_{3}$. 


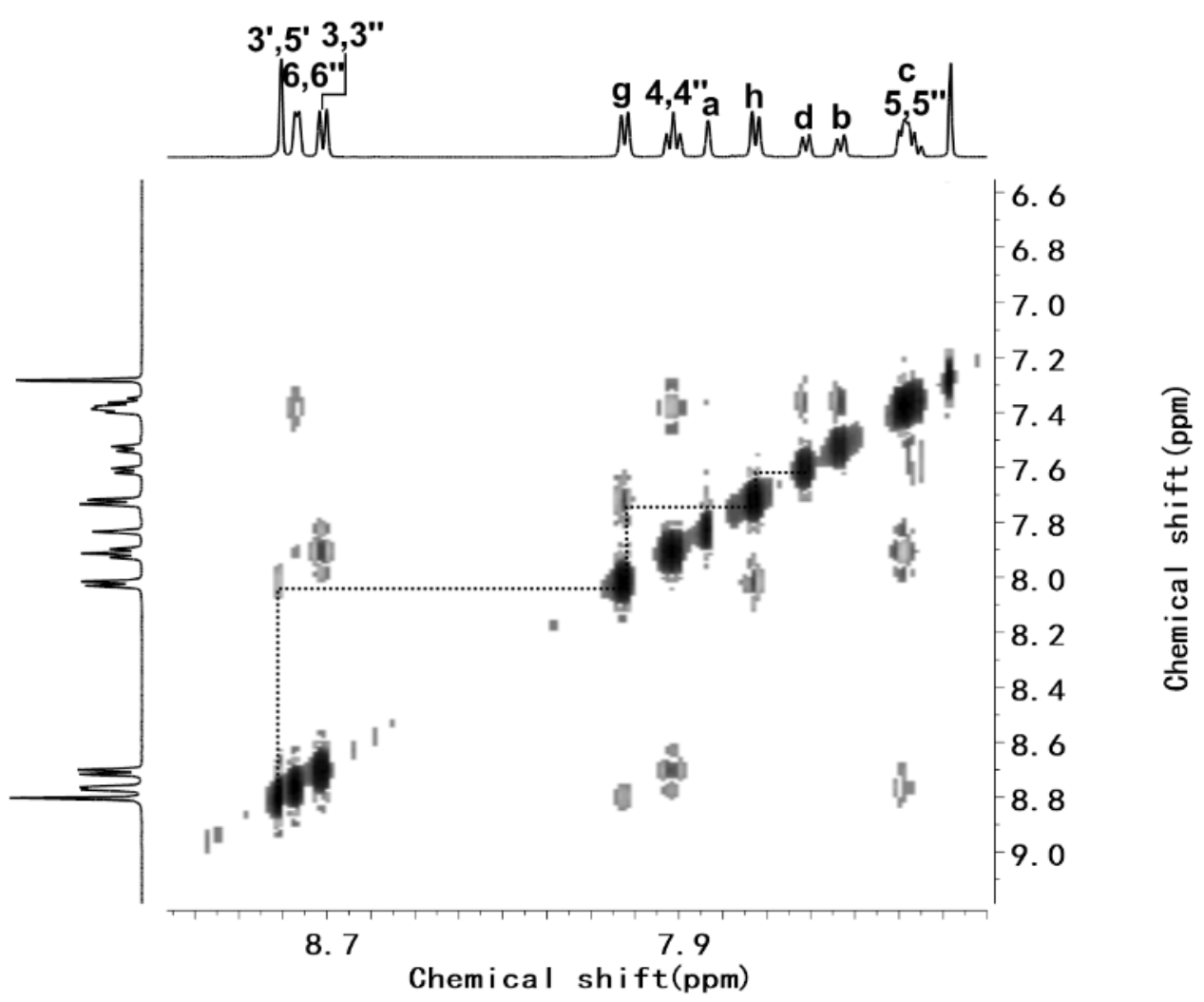

Figure S19: 2D NOESY spectrum (500 MHz) of compound $\mathbf{S 8}$ in $\mathrm{CDCl}_{3}$.

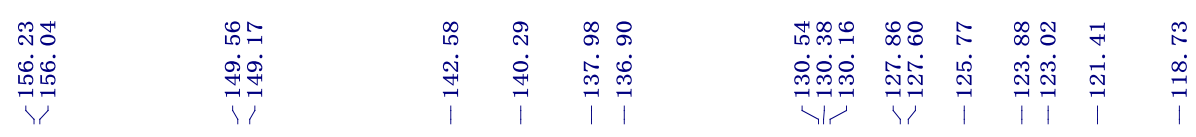

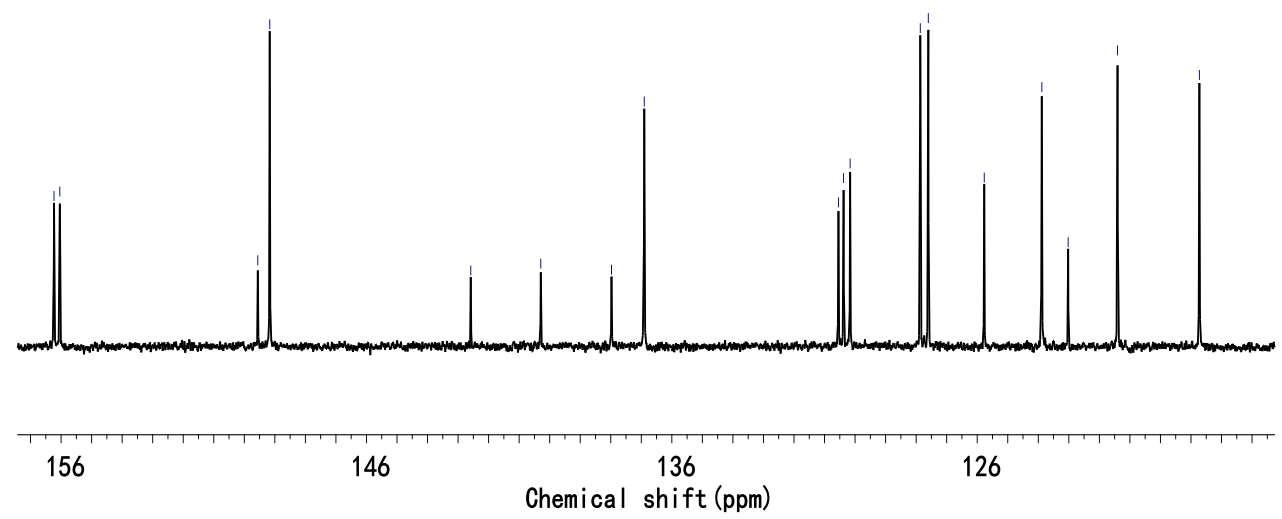

Figure S20: ${ }^{13} \mathrm{C}$ NMR spectrum (126 MHz) of compound $\mathbf{S 8}$ in $\mathrm{CDCl}_{3}$. 


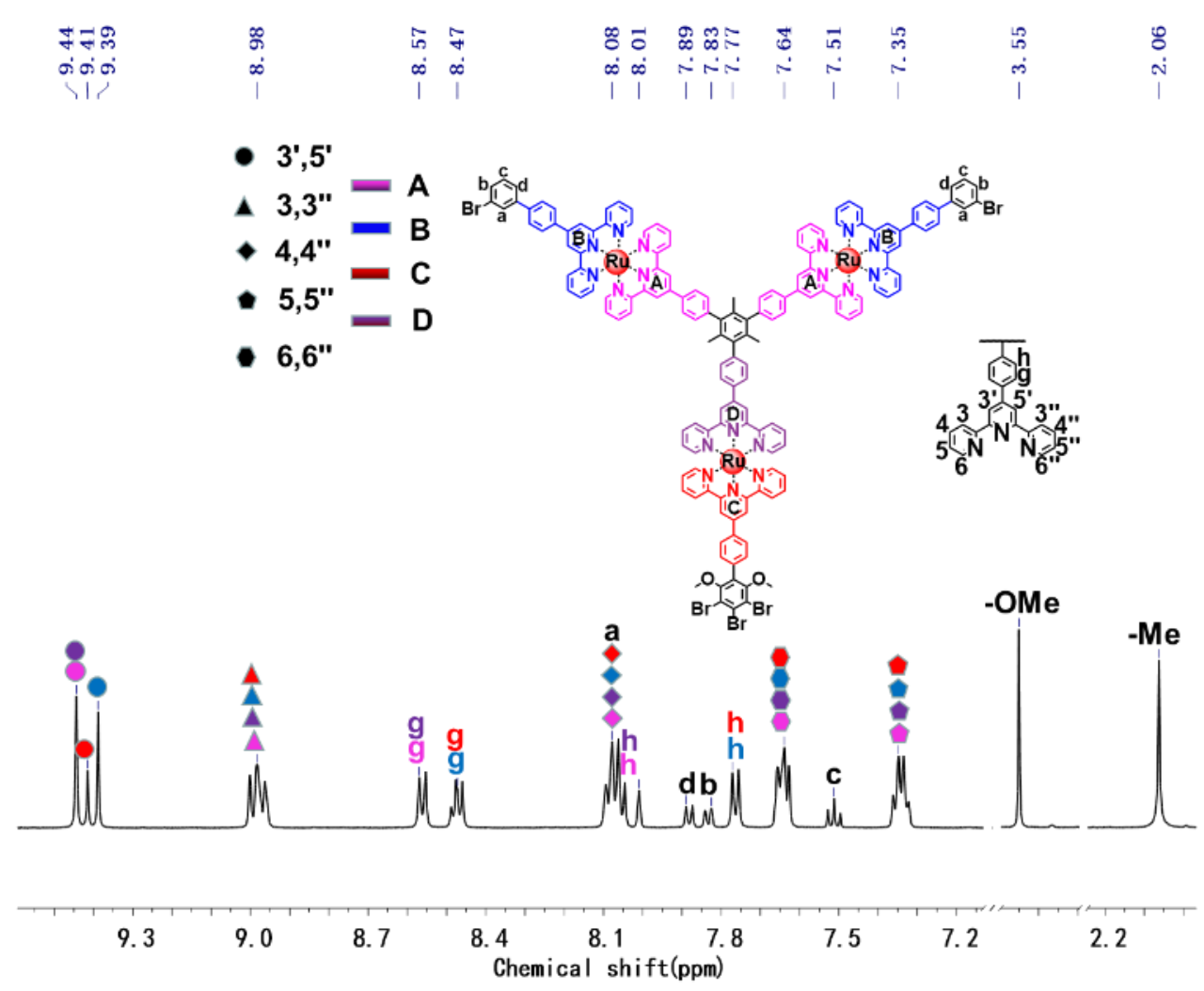

Figure S21: ${ }^{1} \mathrm{H}$ NMR spectrum (500 MHz) of complex 5 in MeOD.

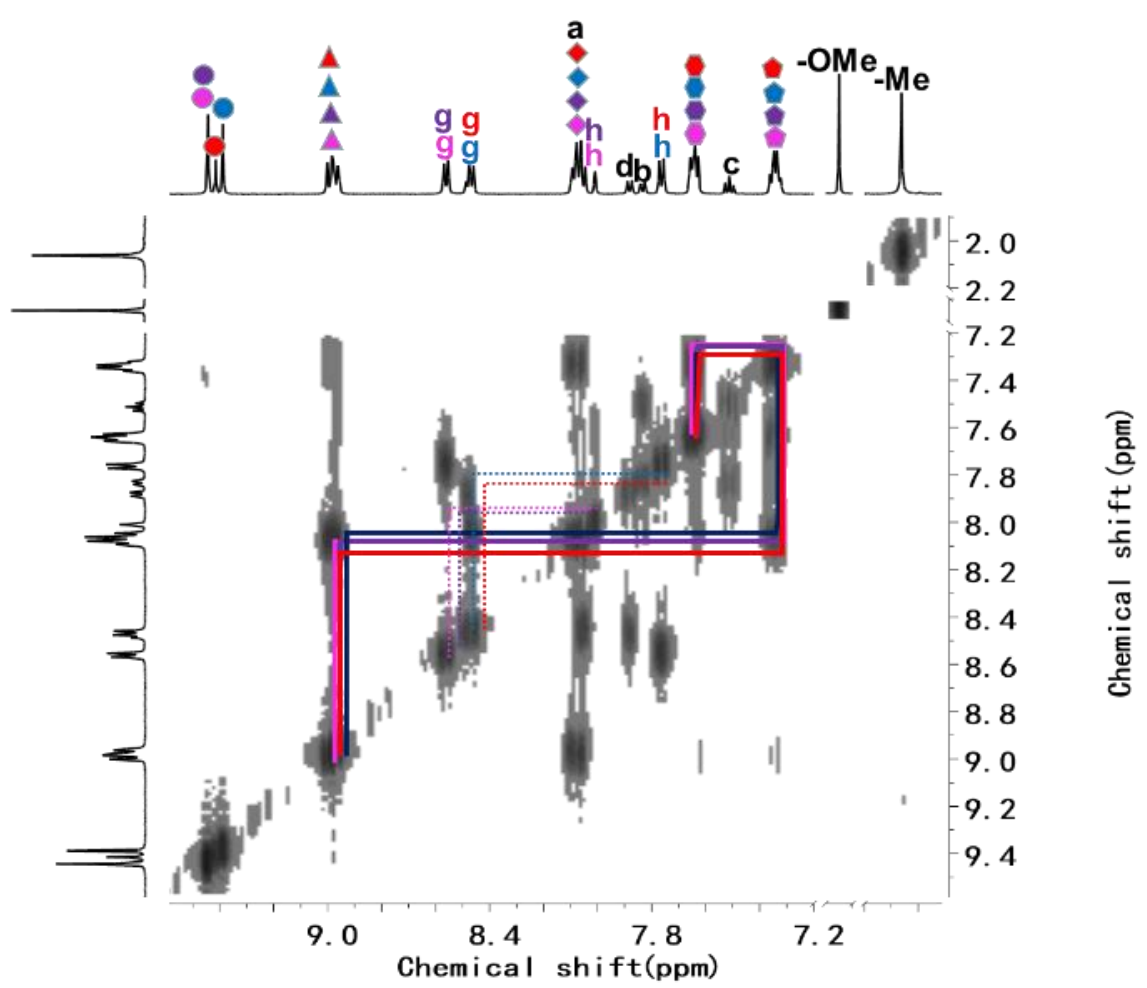

Figure S22: 2D COSY spectrum (500 MHz) of complex 5 in MeOD. 


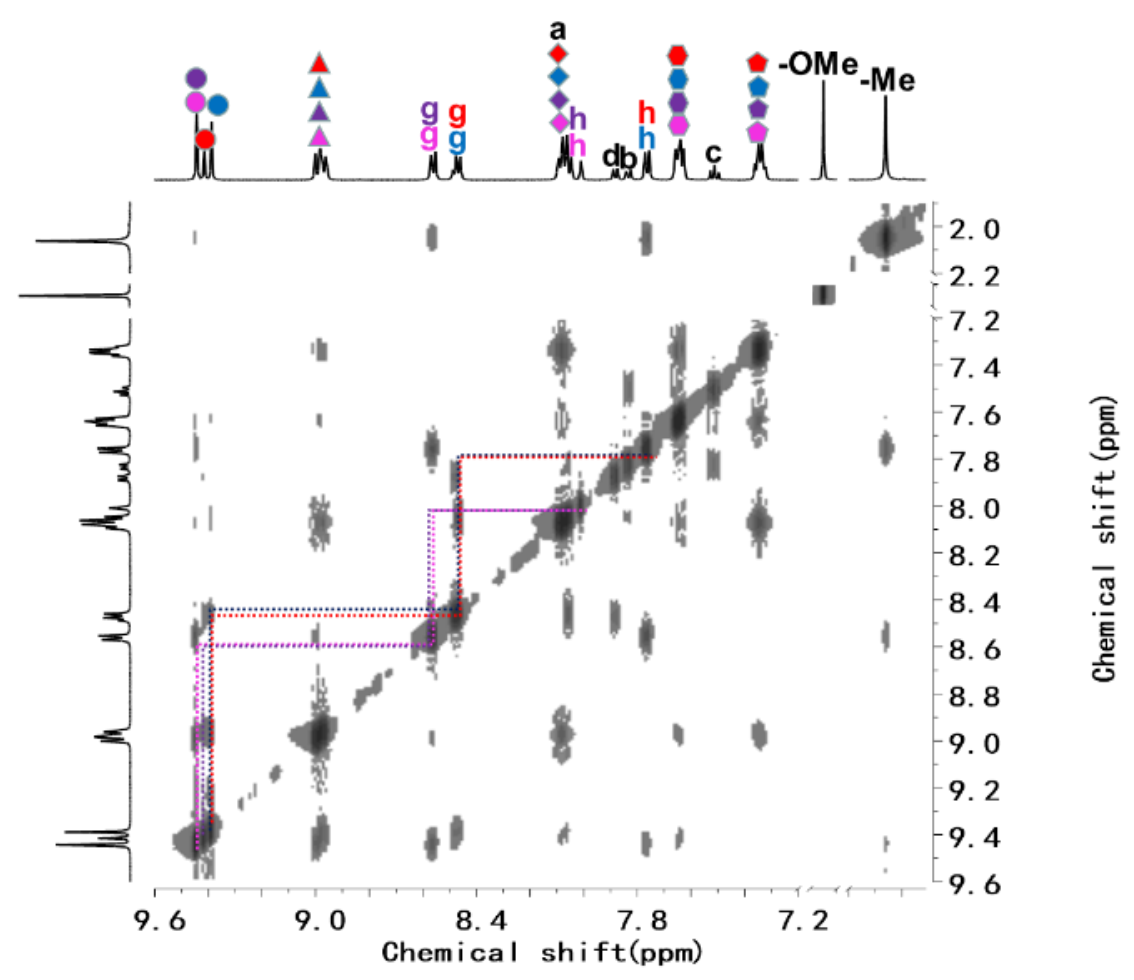

Figure S23: 2D NOESY spectrum (500 MHz) of complex 5 in MeOD.

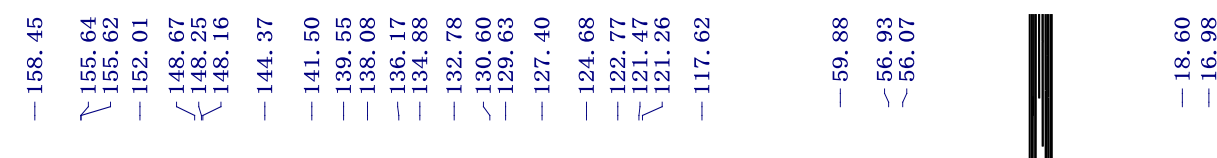

$$
\begin{array}{ll}
8 & \infty \\
8 & 0 \\
\infty & 0 \\
0 & 0
\end{array}
$$

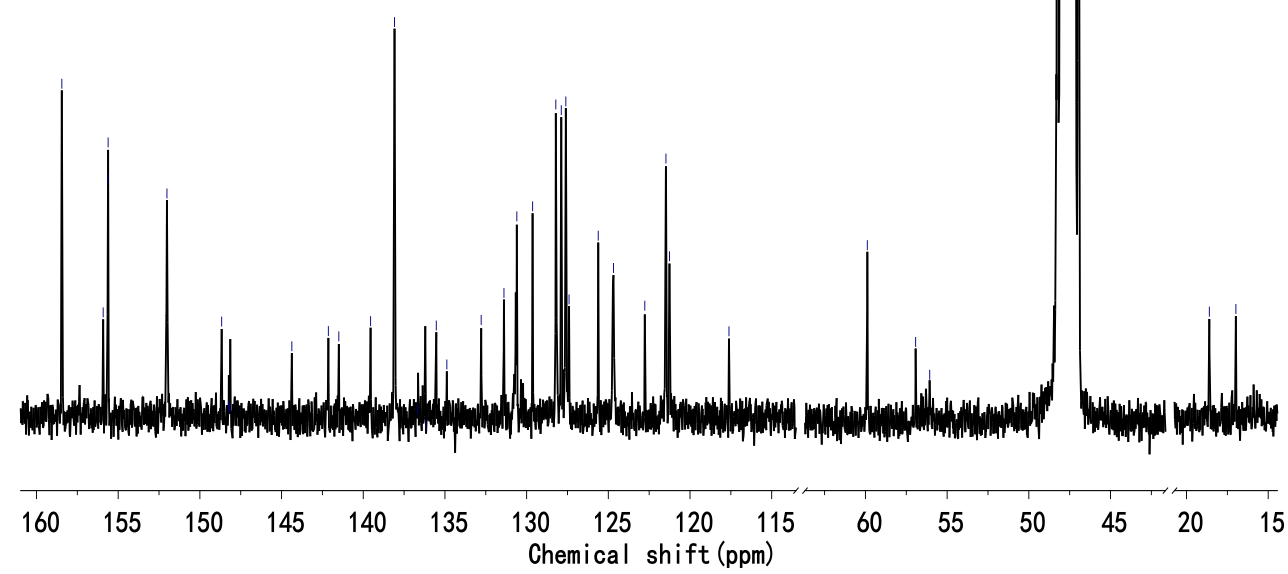

Figure S24: ${ }^{13} \mathrm{C}$ NMR spectrum (101 MHz) of complex 5 in MeOD. 


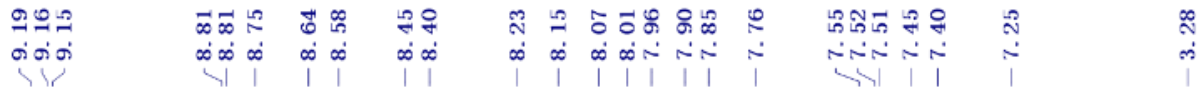
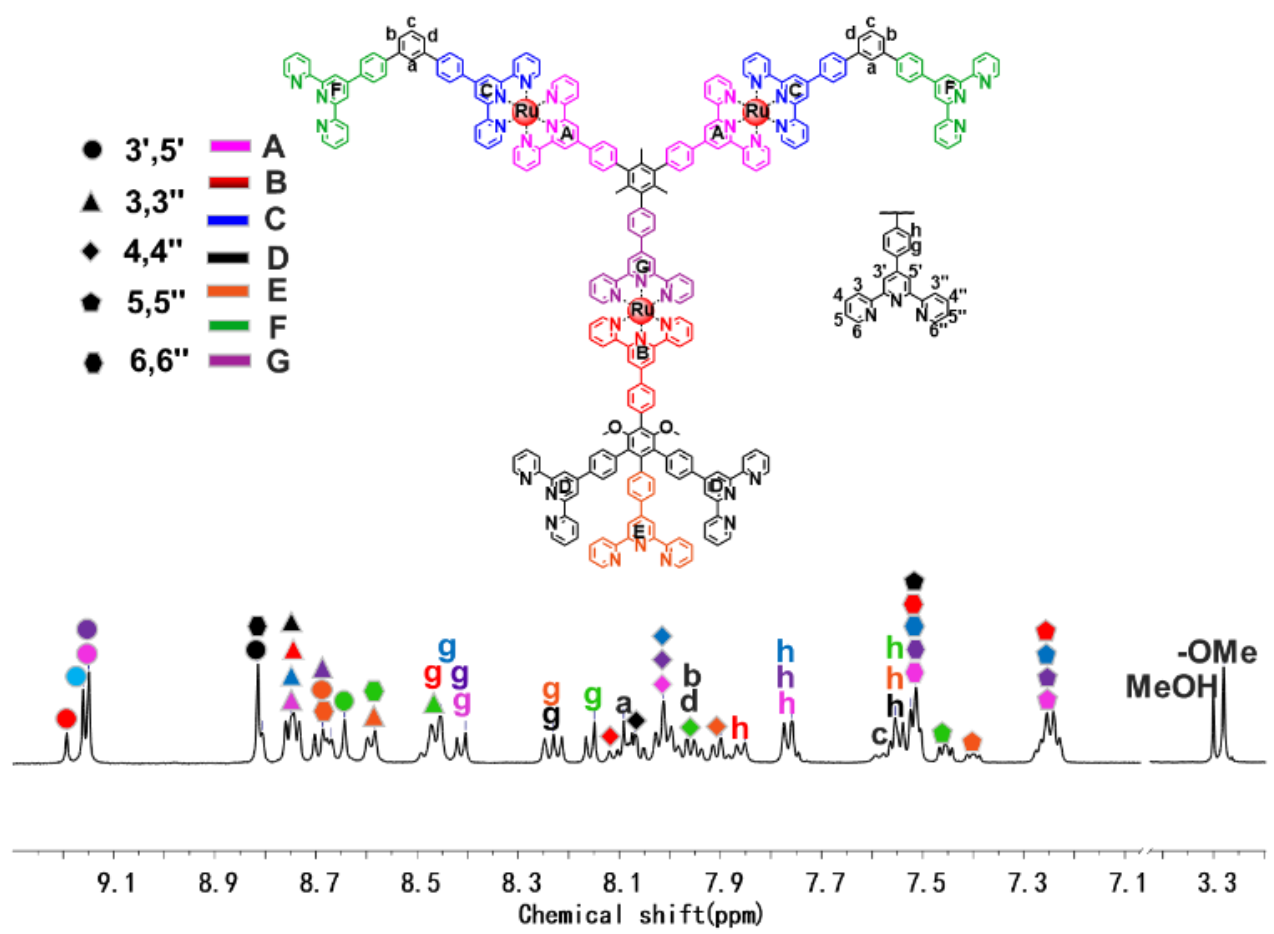

Figure S25: ${ }^{1} \mathrm{H}$ NMR spectrum $(500 \mathrm{MHz})$ of ligand $\mathbf{L A}$ in $\mathrm{CD}_{3} \mathrm{CN}$.

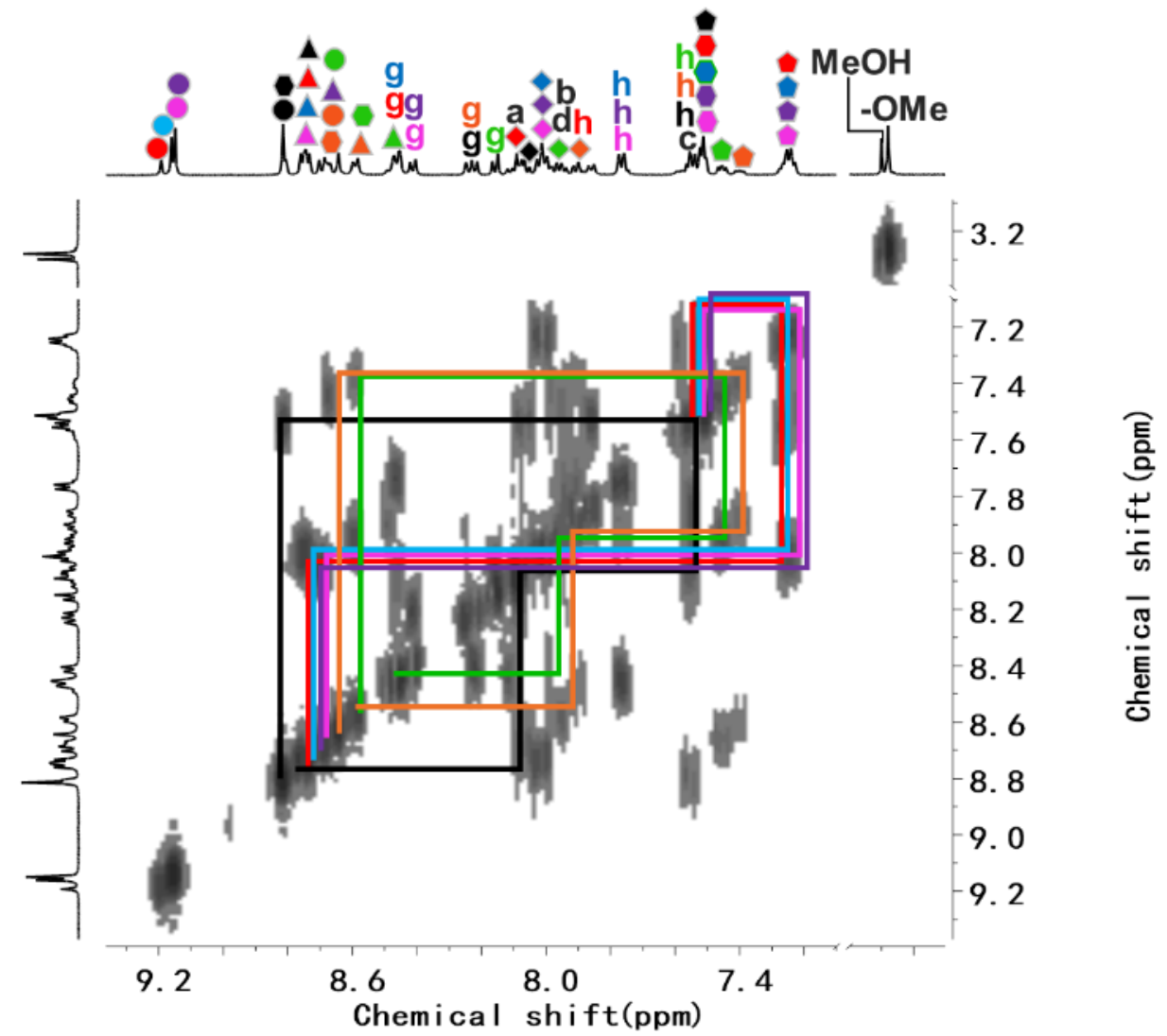

Figure S26: $2 \mathrm{D}$ COSY spectrum $(500 \mathrm{MHz})$ of ligand $\mathbf{L A}$ in $\mathrm{CD}_{3} \mathrm{CN}$. 


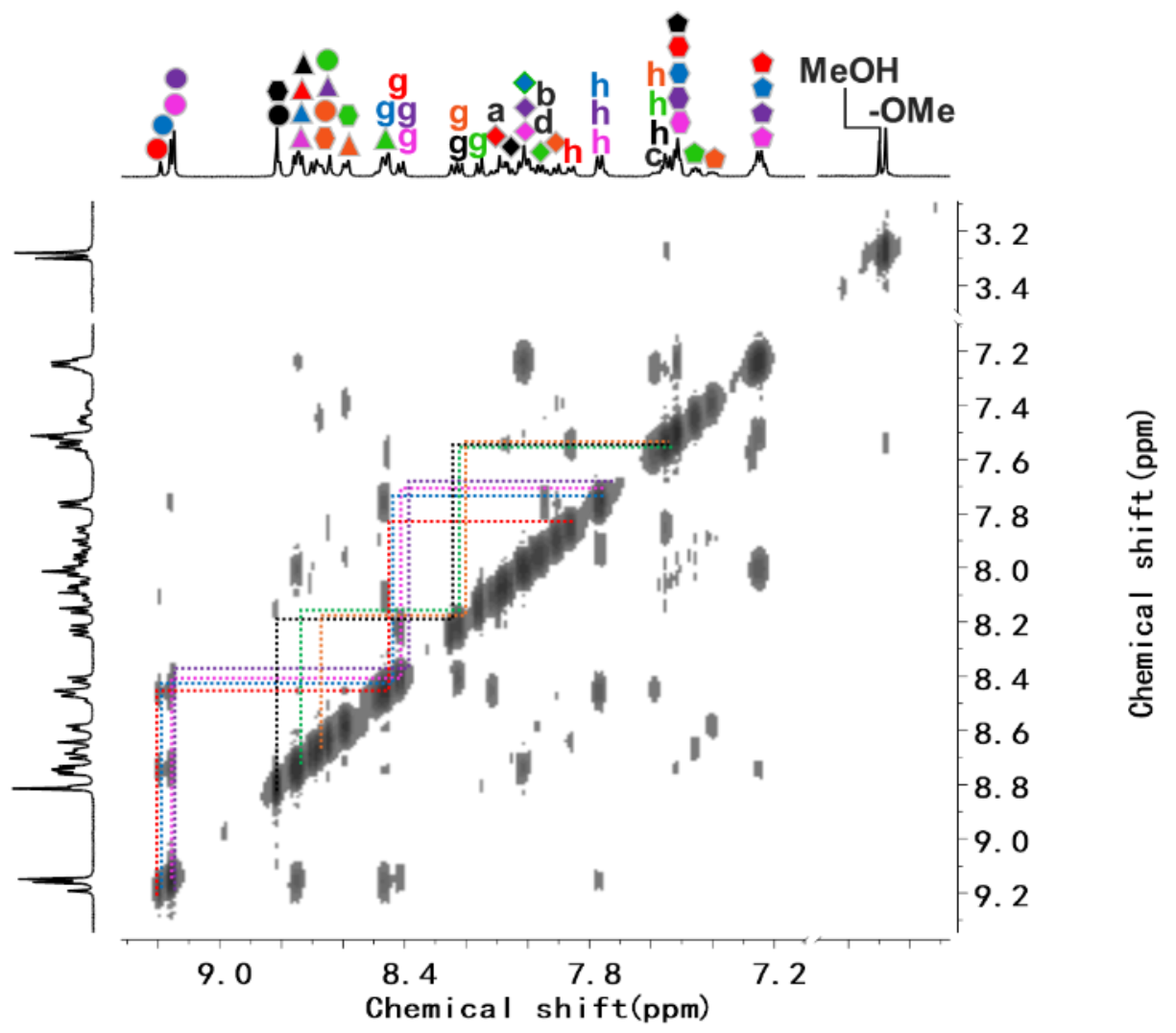

Figure S27: 2D NOESY spectrum (500 MHz) of ligand $\mathbf{L A}$ in $\mathrm{CD}_{3} \mathrm{CN}$.

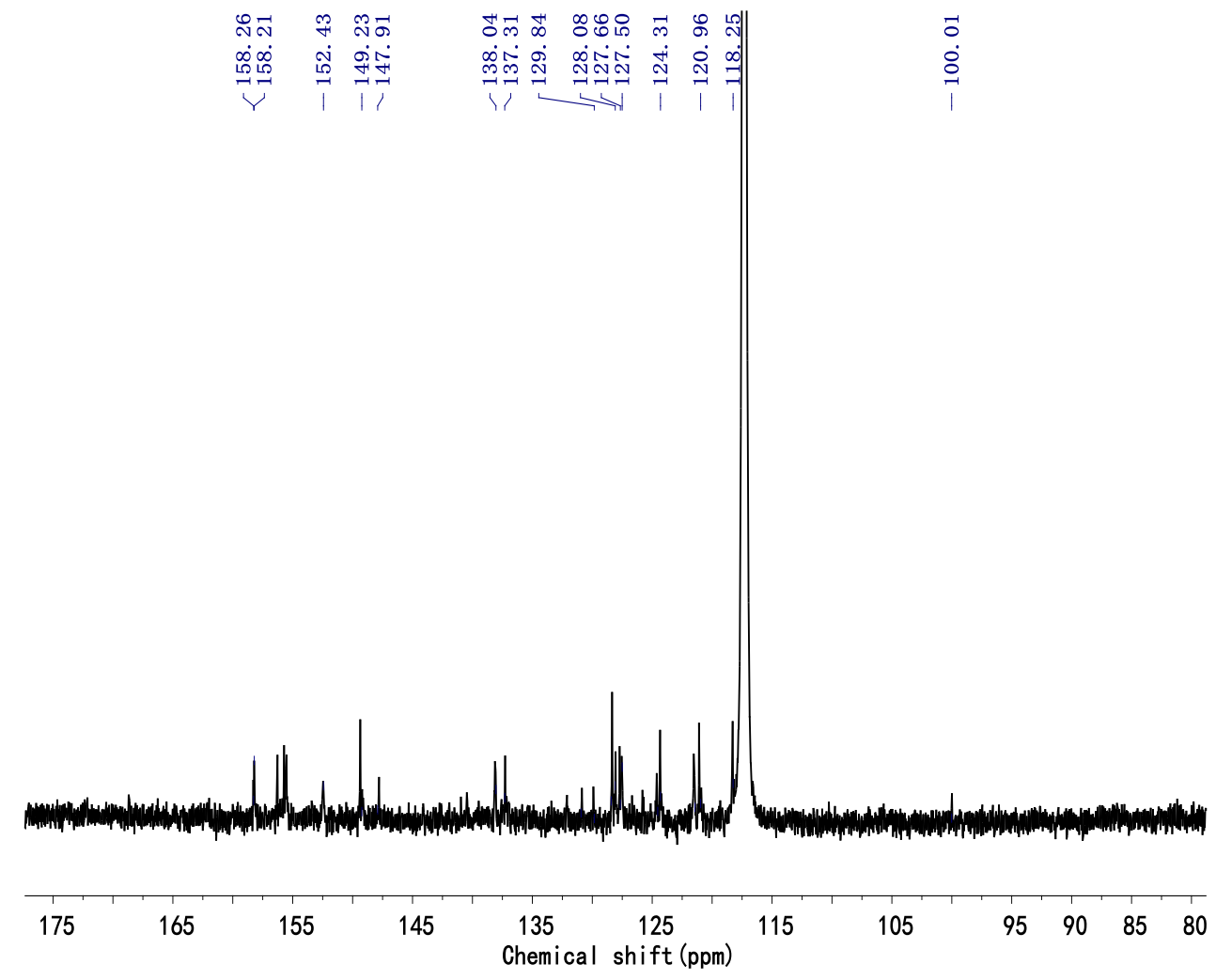

Figure S28: ${ }^{13} \mathrm{C}$ NMR spectrum (101 MHz) of ligand $\mathbf{L A}$ in $\mathrm{CD}_{3} \mathrm{CN}$. 

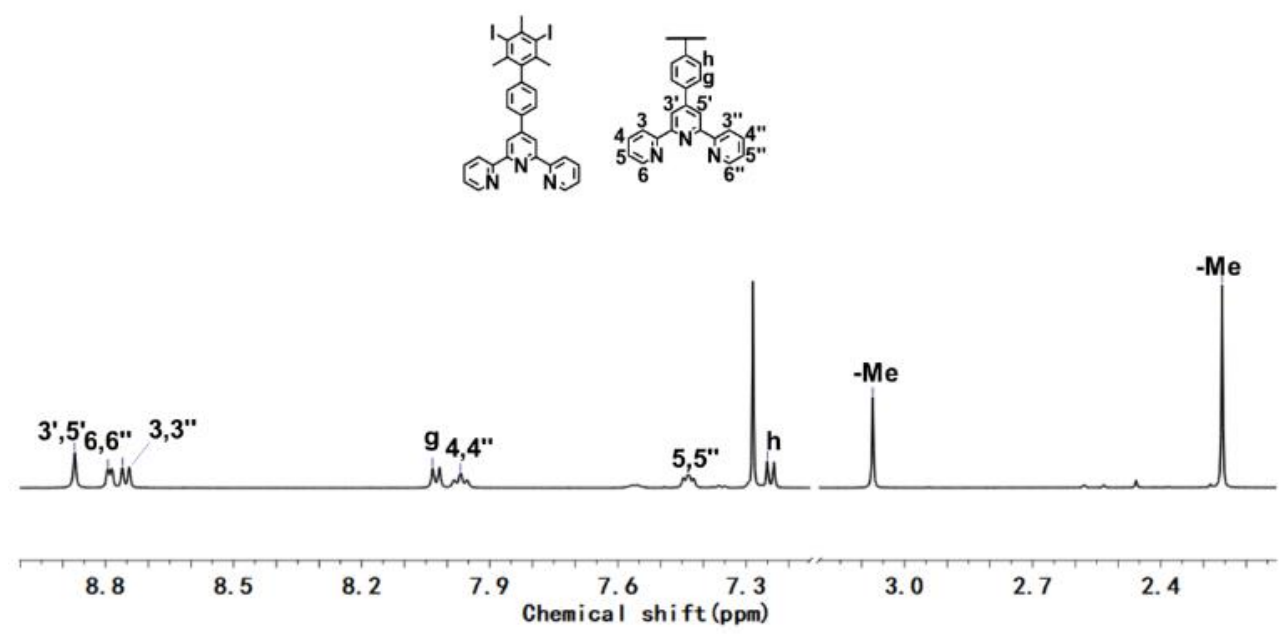

Figure S29: ${ }^{1} \mathrm{H}$ NMR spectrum $(500 \mathrm{MHz})$ of compound $\mathbf{S 9}$ in $\mathrm{CDCl}_{3}$.

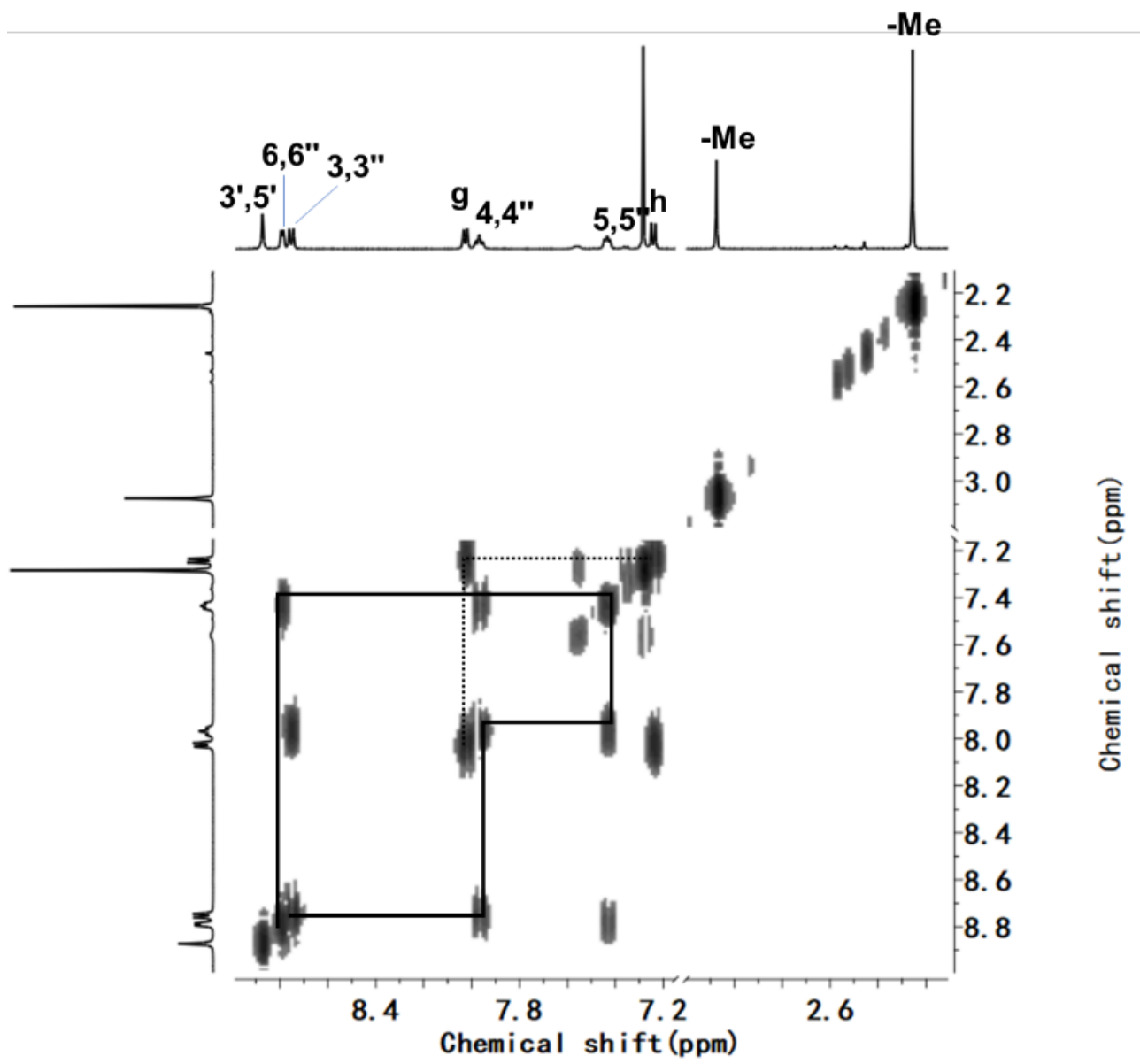

Figure S30: 2D COSY spectrum $(500 \mathrm{MHz})$ of compound $\mathbf{S 9}$ in $\mathrm{CDCl}_{3}$. 


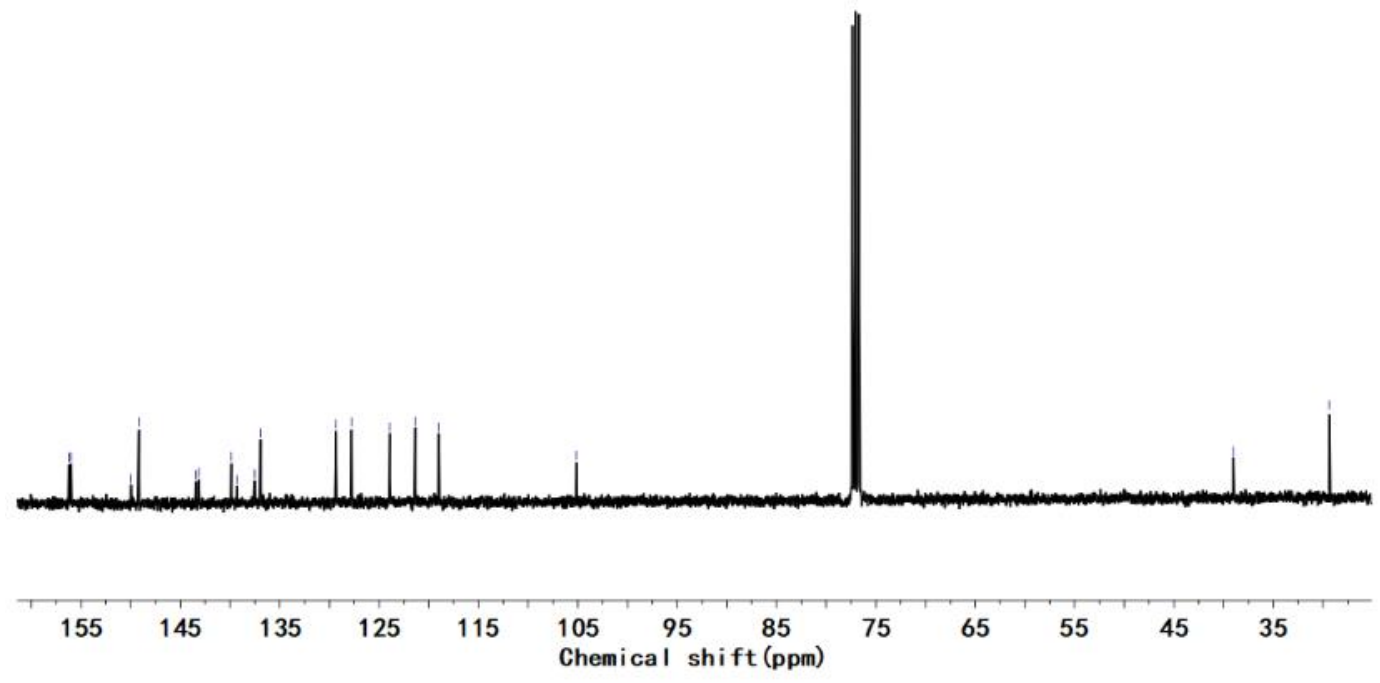

Figure S31: ${ }^{13} \mathrm{C}$ NMR spectrum $(101 \mathrm{MHz})$ of compound $\mathbf{S 9}$ in $\mathrm{CDCl}_{3}$.

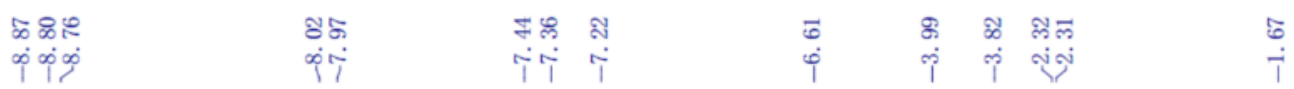

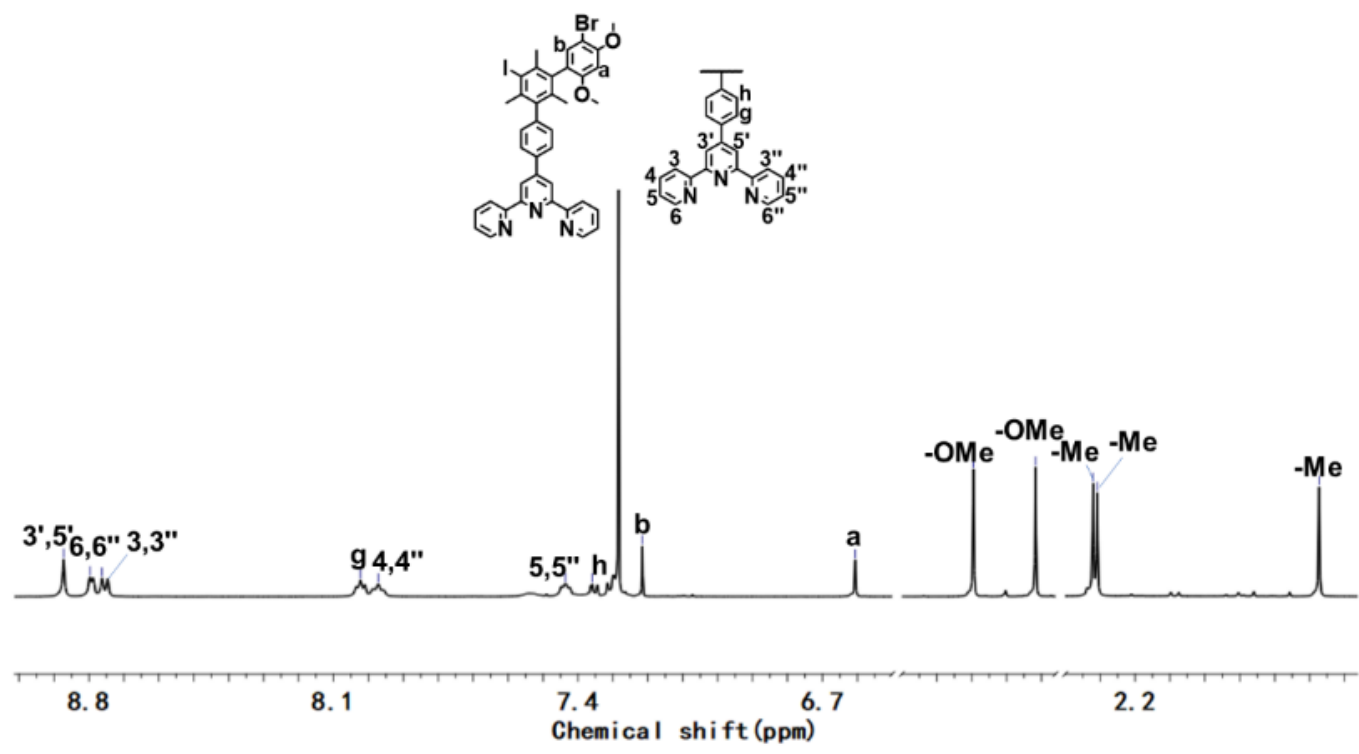

Figure S32: ${ }^{1} \mathrm{H}$ NMR spectrum $(500 \mathrm{MHz})$ of compound $\mathbf{S 1 1}$ in $\mathrm{CDCl}_{3}$. 


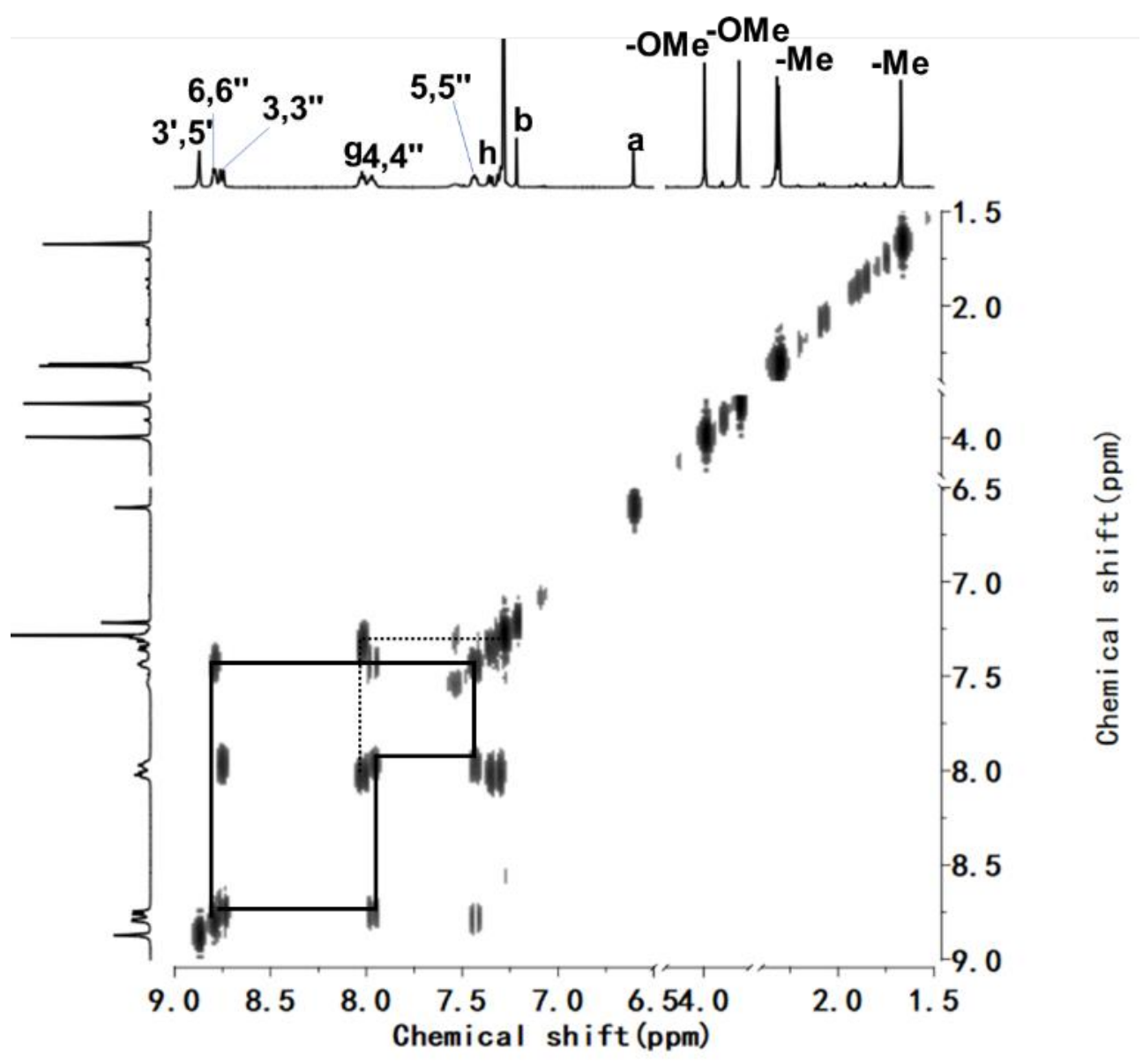

Figure S33: 2D COSY spectrum (500 MHz) of compound $\mathbf{S 1 1}$ in $\mathrm{CDCl}_{3}$.

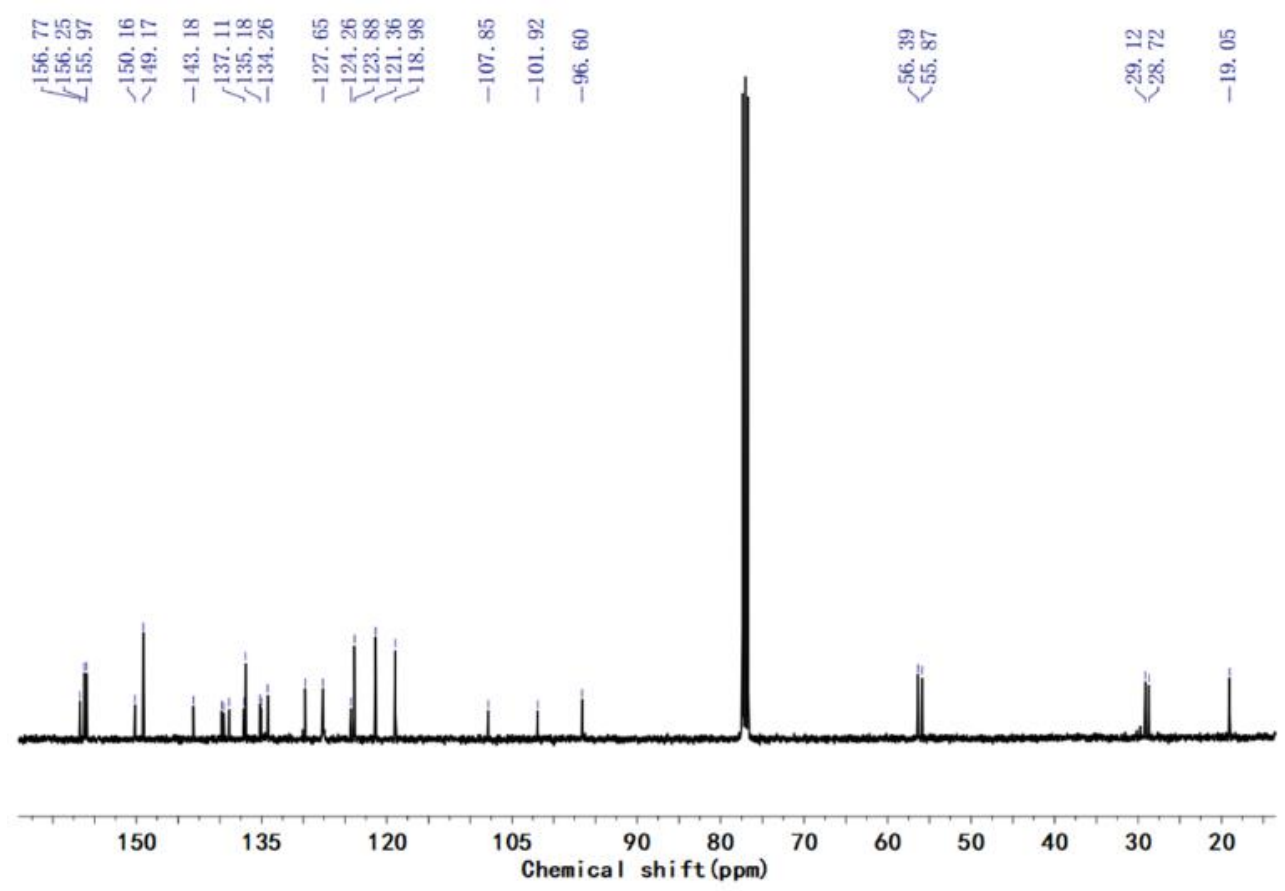

Figure S34: ${ }^{13} \mathrm{C}$ NMR spectrum $(101 \mathrm{MHz})$ of compound $\mathbf{S 1 1}$ in $\mathrm{CDCl}_{3}$. 


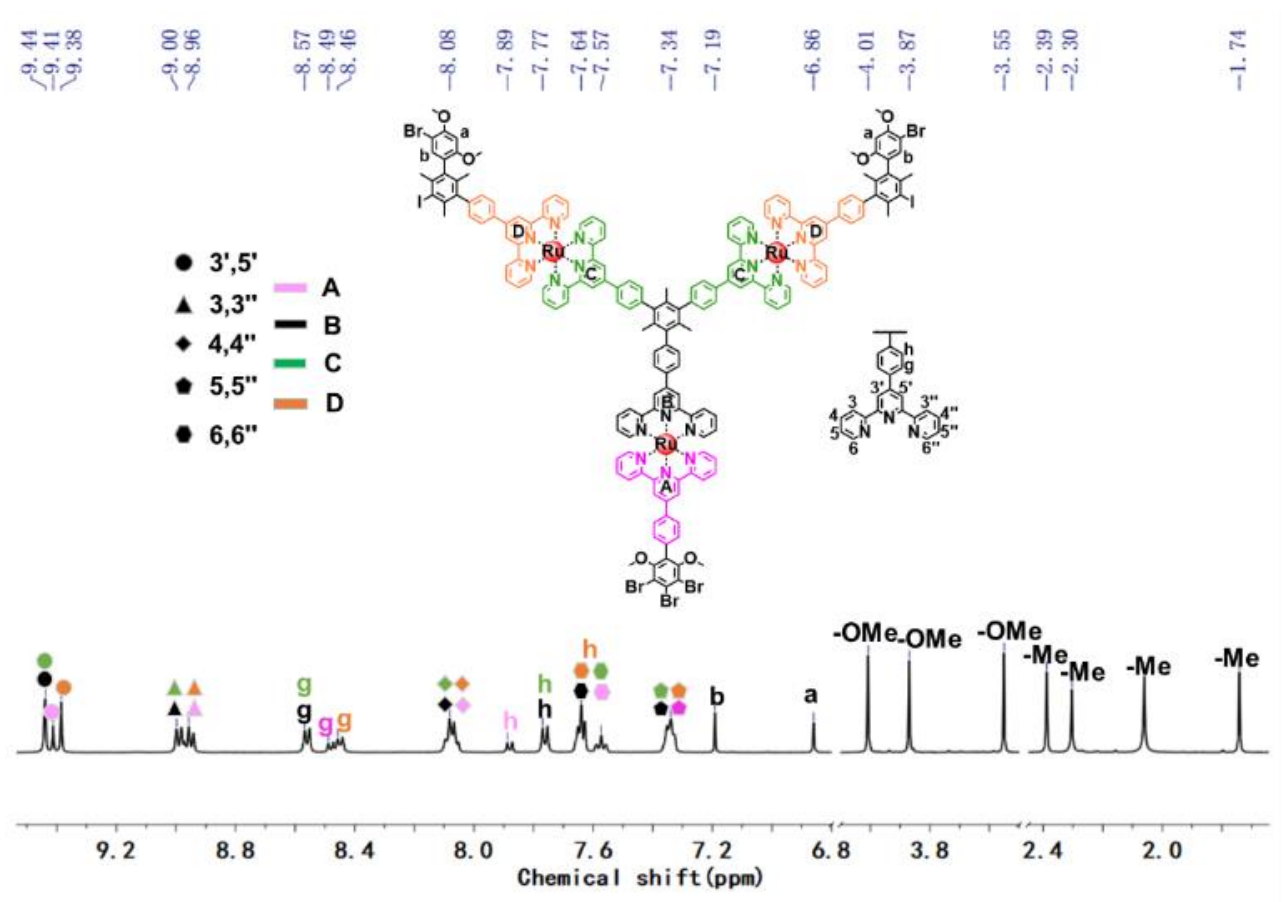

Figure S35: ${ }^{1} \mathrm{H}$ NMR spectrum (500 MHz) of complex 8 in MeOD.

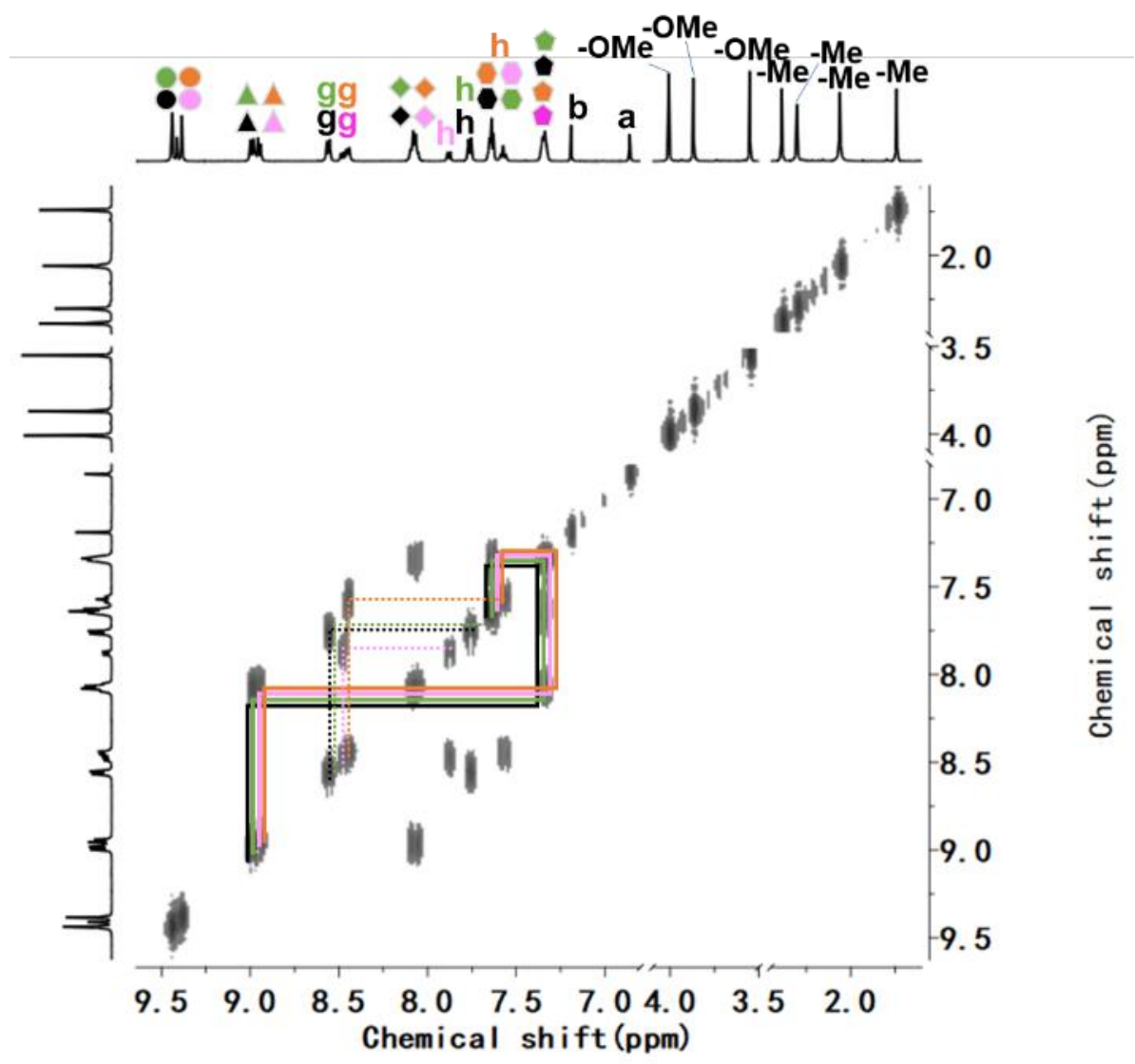

Figure S36: 2D COSY spectrum (500 MHz) of complex 8 in MeOD. 


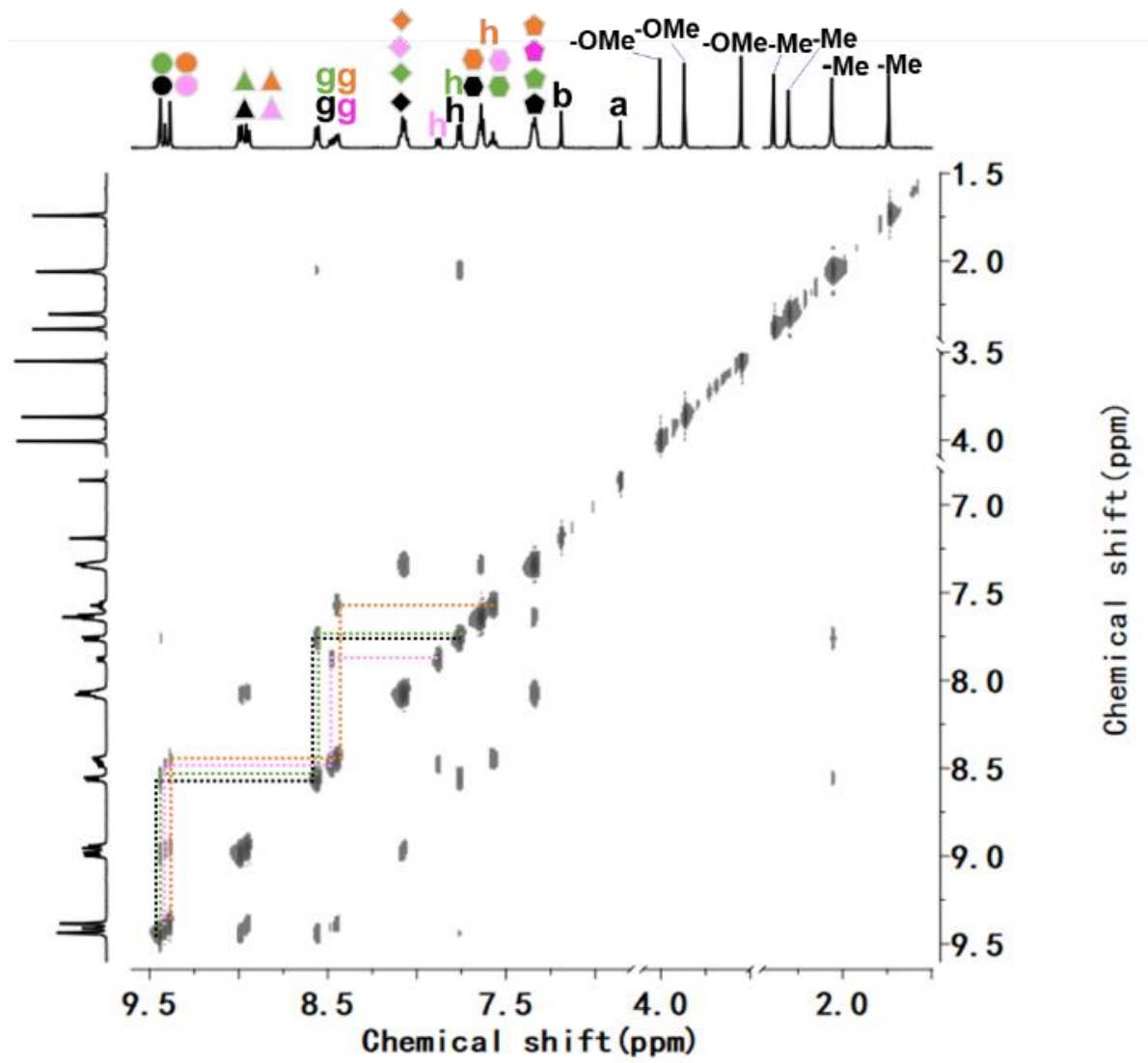

Figure S37: 2D NOESY spectrum (500 MHz) of complex 8 in MeOD.

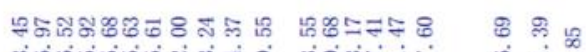

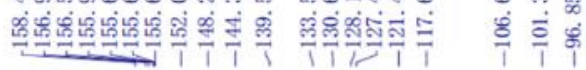

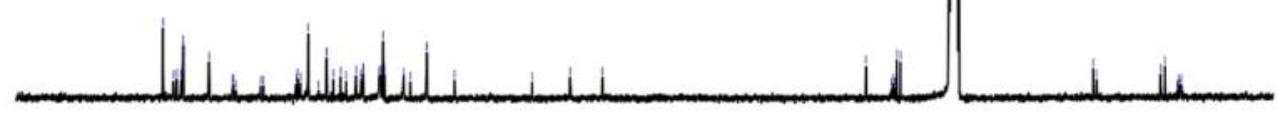

$\begin{array}{lllllllllllllllll}170 & 160 & 150 & 140 & 130 & 120 & 110 & 100 & 90 & 80 & 70 & 60 & 50 & 40 & 30 & 20 & 10\end{array}$

Figure S38: ${ }^{13} \mathrm{C}$ NMR spectrum (101 MHz) of complex 8 in MeOD. 


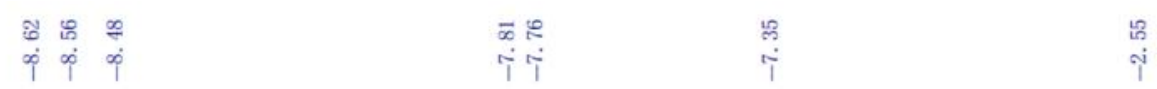
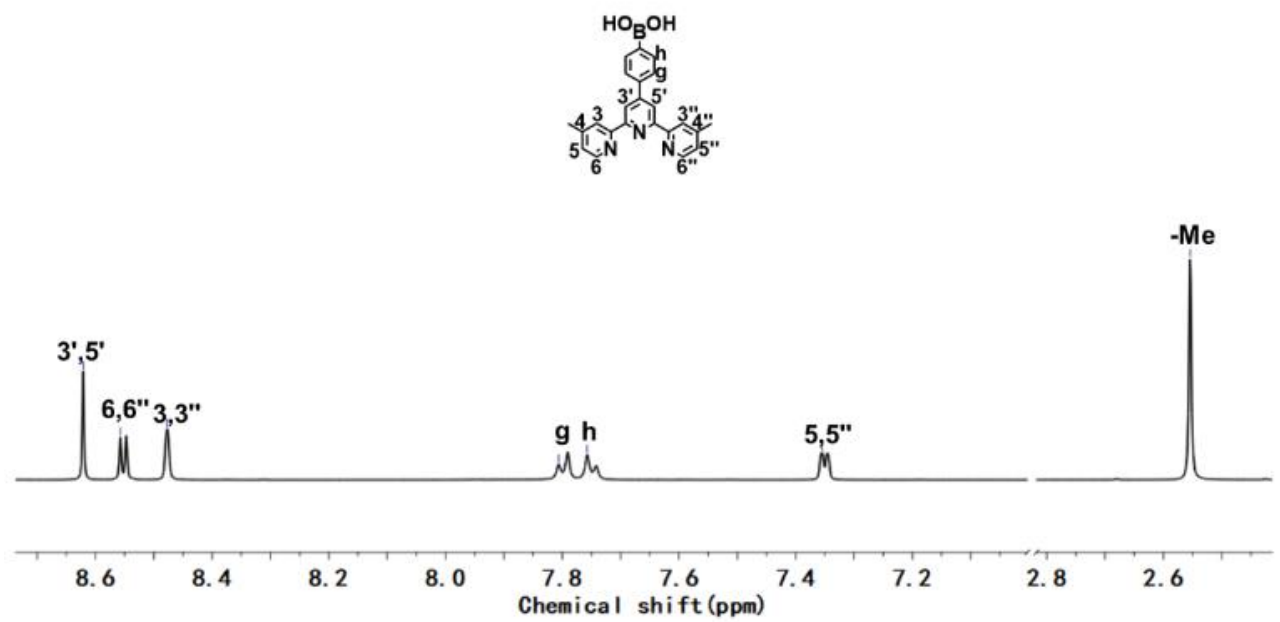

Figure S39: ${ }^{1} \mathrm{H}$ NMR spectrum $(500 \mathrm{MHz})$ of compound 9 in MeOD.

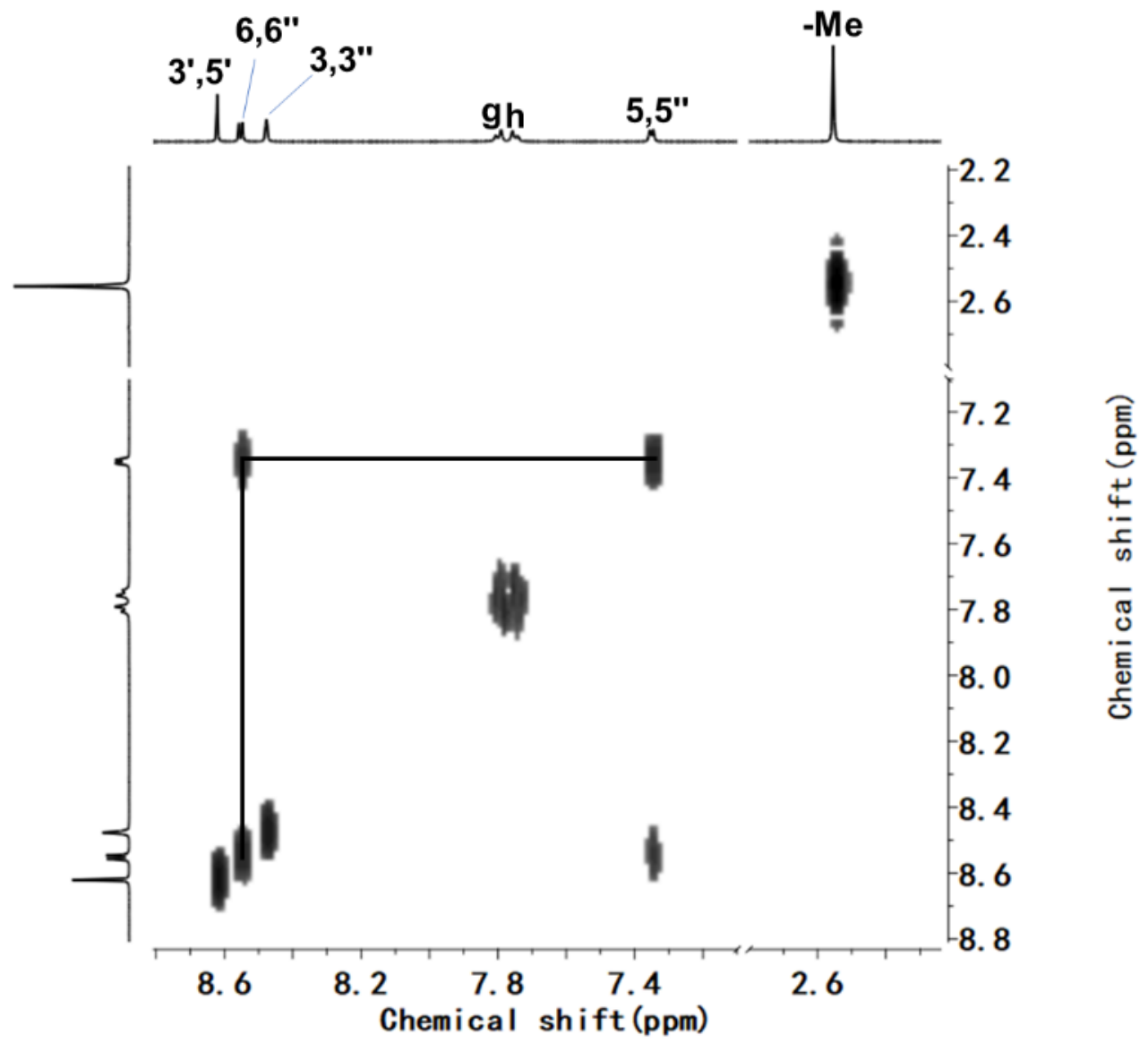

Figure S40: 2D COSY spectrum (500 MHz) of compound 9 in MeOD. 


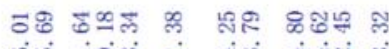

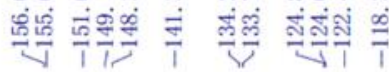
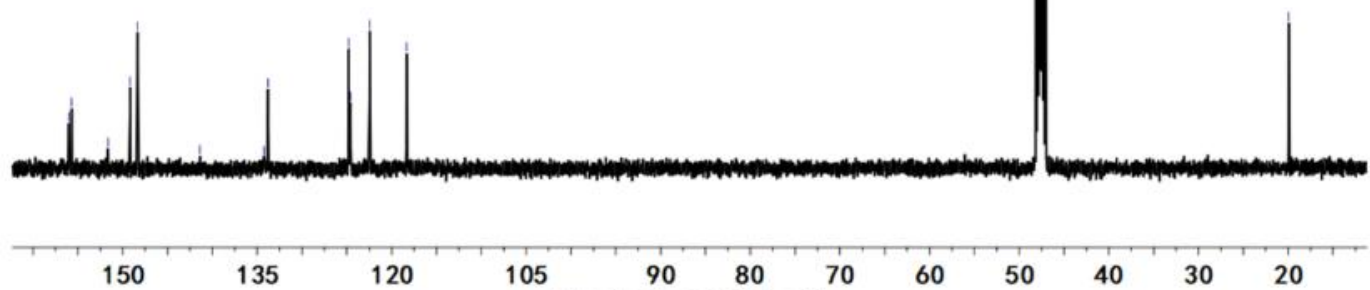

120

105

$90 \quad 80$

Chemical shift (ppm)

Figure S41: ${ }^{13} \mathrm{C}$ NMR spectrum (126 MHz) of compound 9 in MeOD.
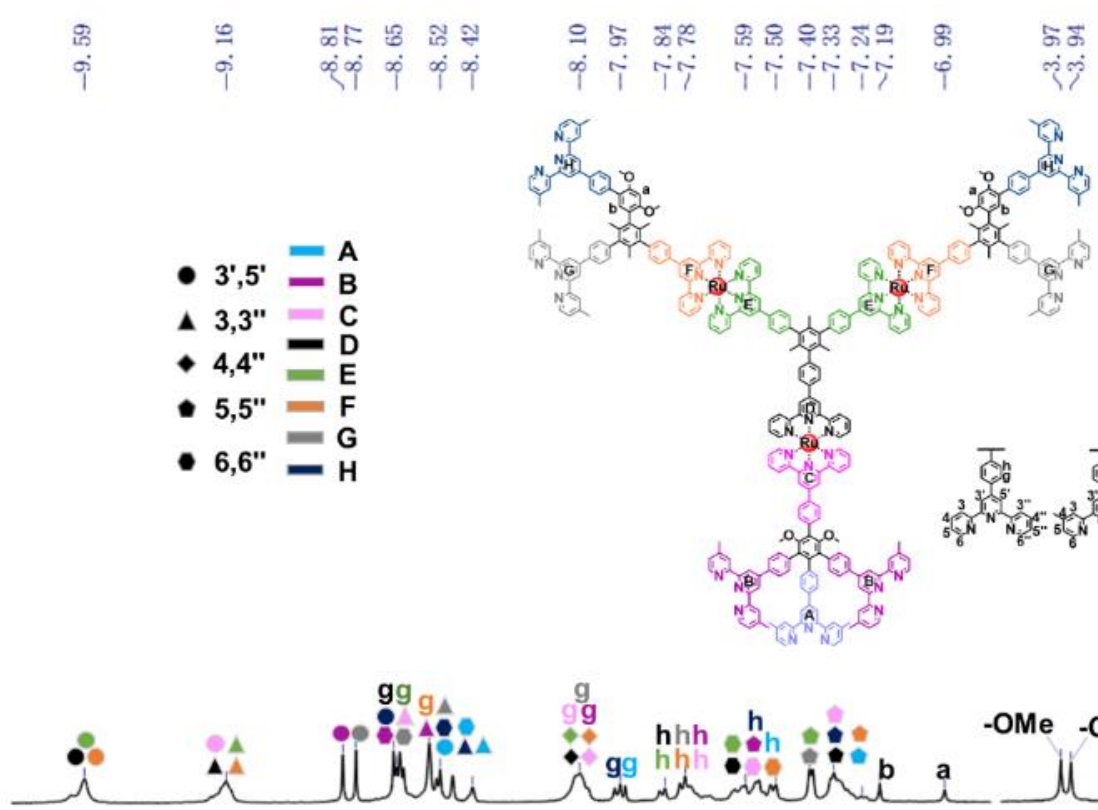

-OMe -OMe
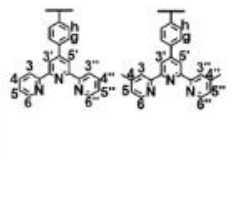

a
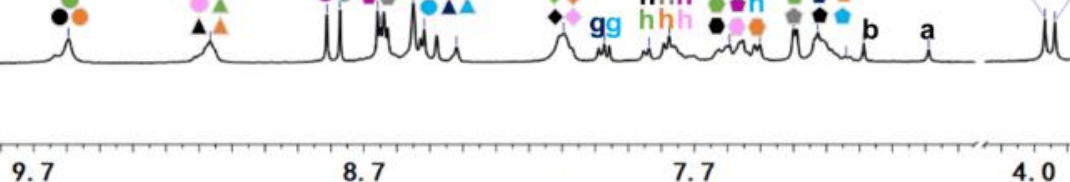

8. 7

7. 7

Chemical shift (ppm)

Figure S42: ${ }^{1} \mathrm{H}$ NMR spectrum (500 MHz) of ligand $\mathbf{L B}$ in DMSO. 


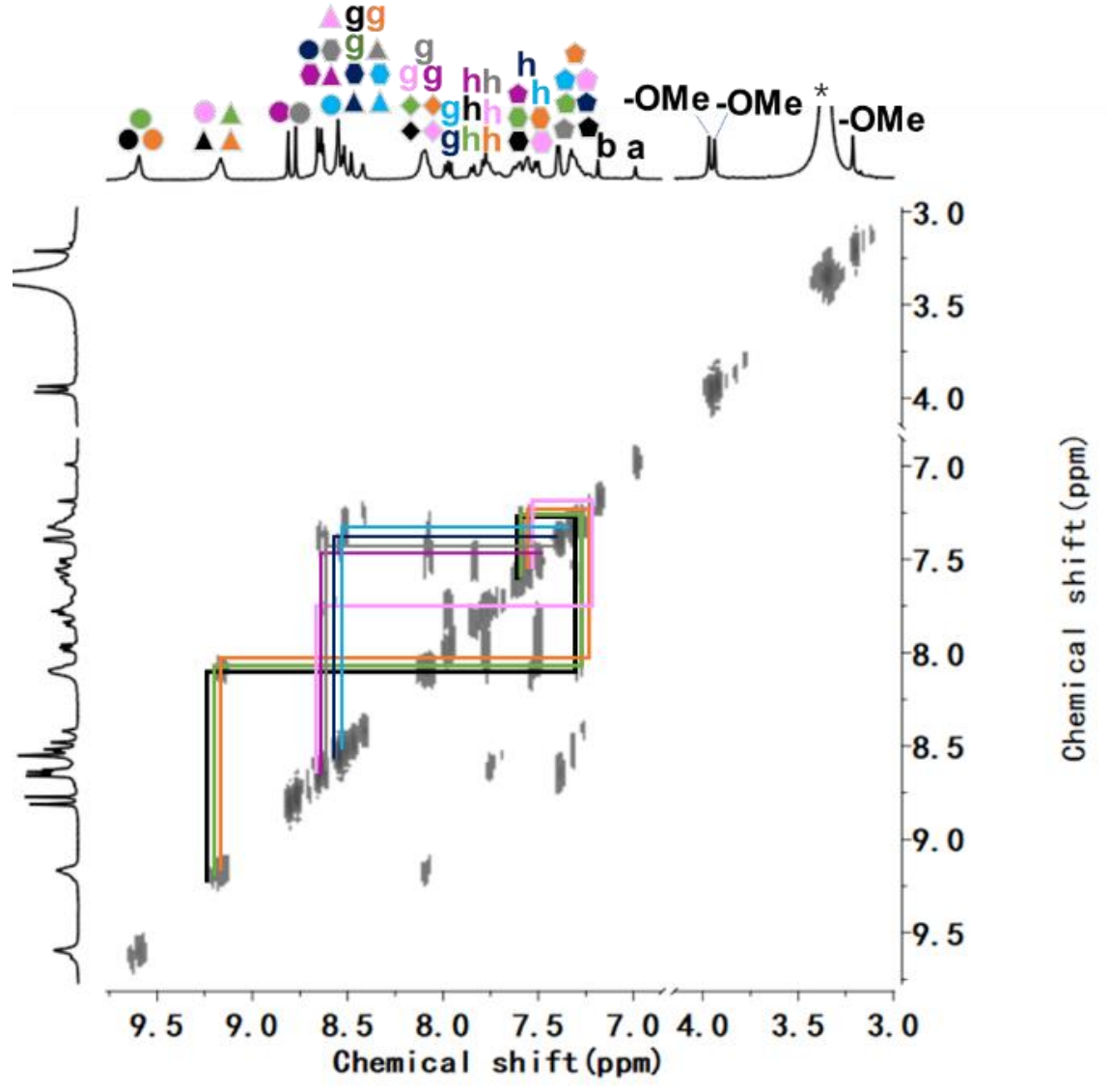

Figure S43: 2D COSY spectrum (500 MHz) of ligand LB in DMSO.

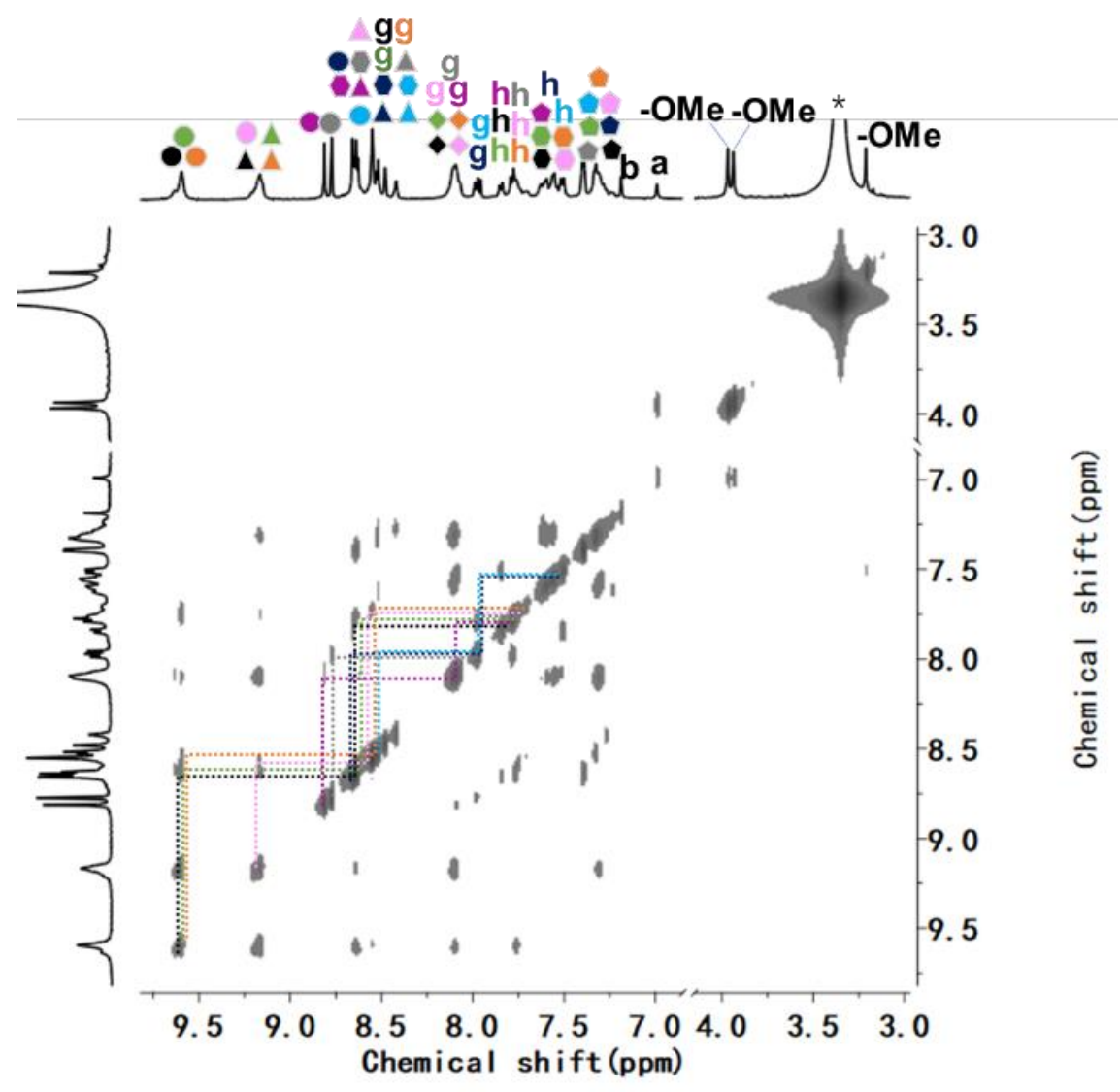

Figure S44: 2D NOESY spectrum (500 MHz) of ligand LB in DMSO. 

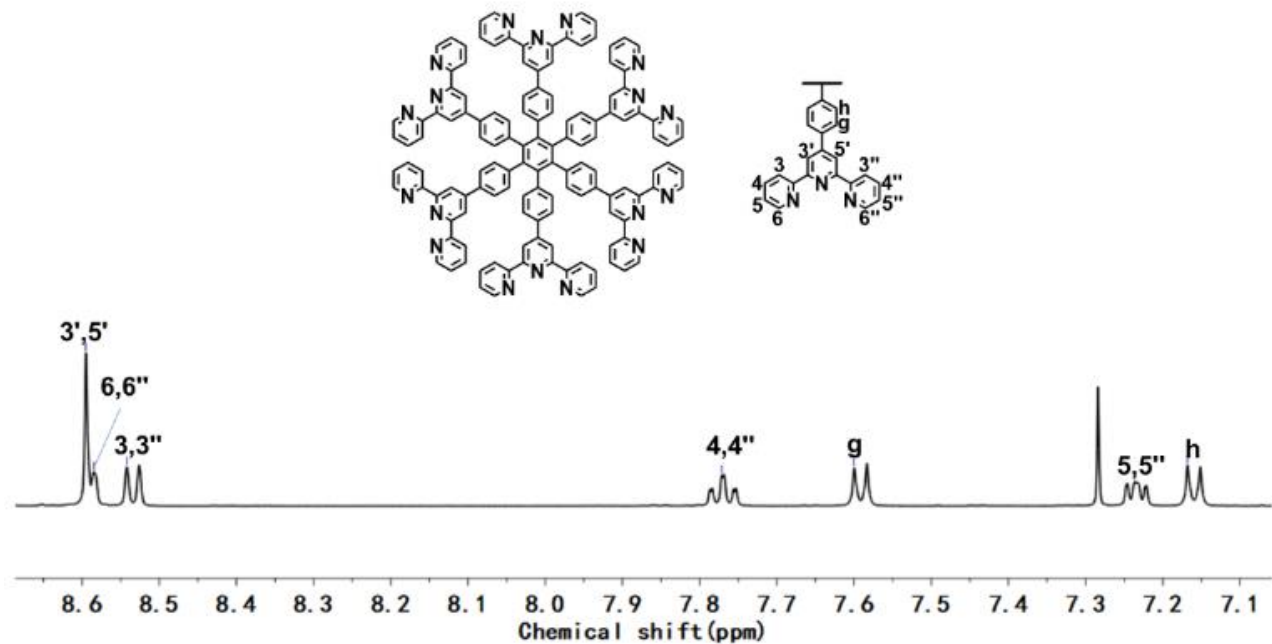

Figure S45: ${ }^{1} \mathrm{H}$ NMR spectrum (500 MHz) of ligand $\mathbf{L H}$ in $\mathrm{CDCl}_{3}$.

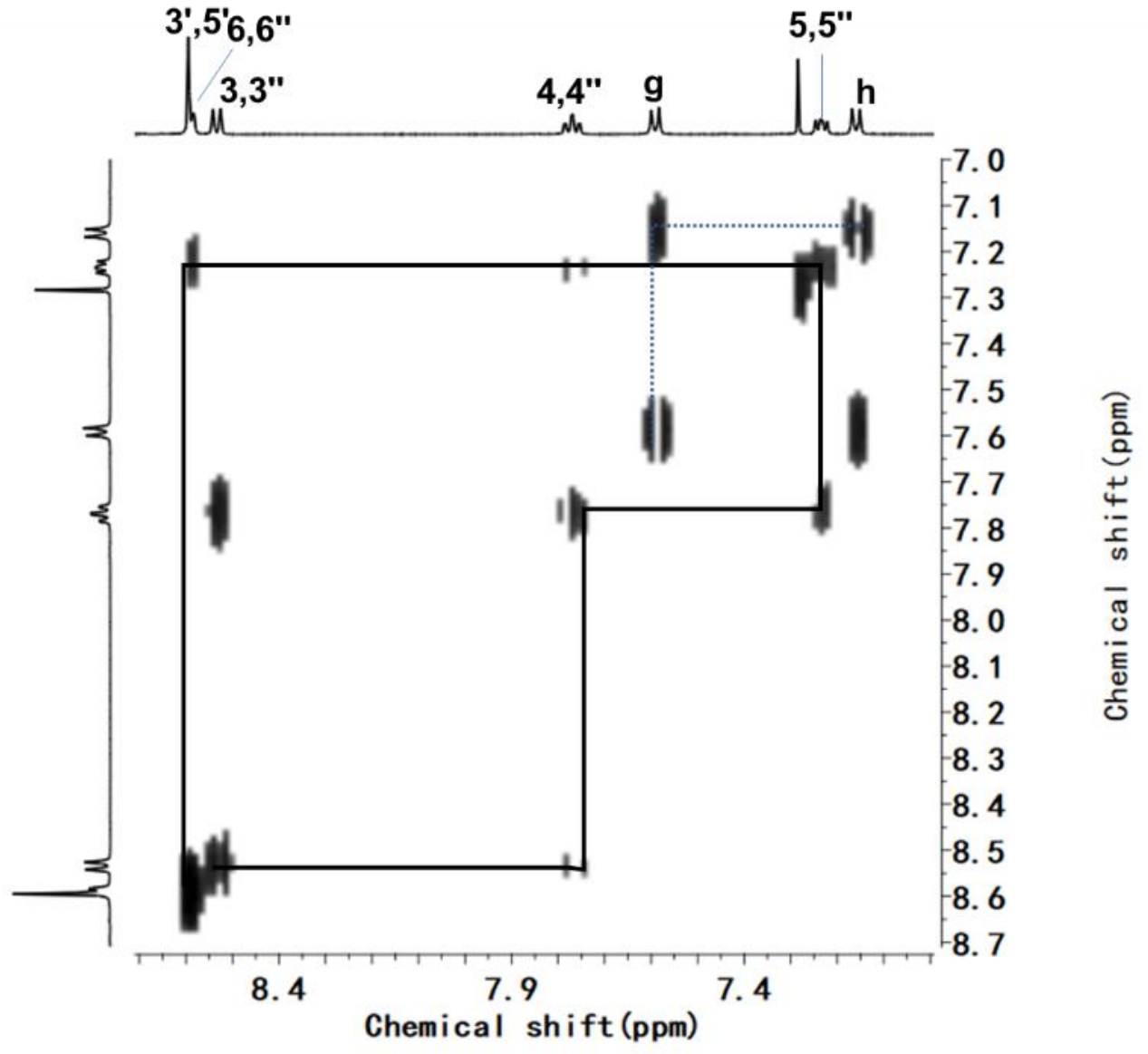

Figure S46: 2D COSY spectrum (500 MHz) of ligand $\mathbf{L H}$ in $\mathrm{CDCl}_{3}$. 


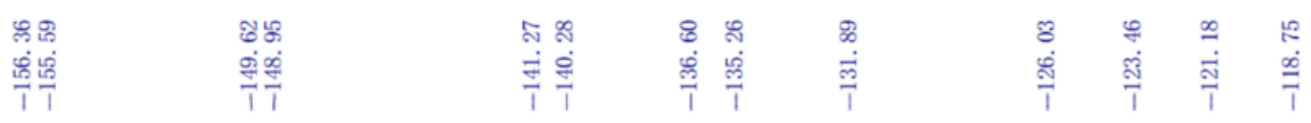

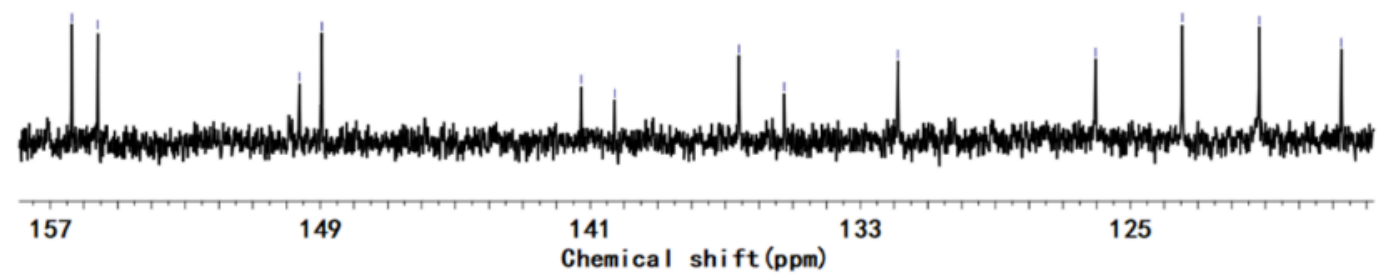

Figure S47: ${ }^{13} \mathrm{C}$ NMR spectrum $(101 \mathrm{MHz})$ of ligand $\mathbf{L H}$ in $\mathrm{CDCl}_{3}$. 


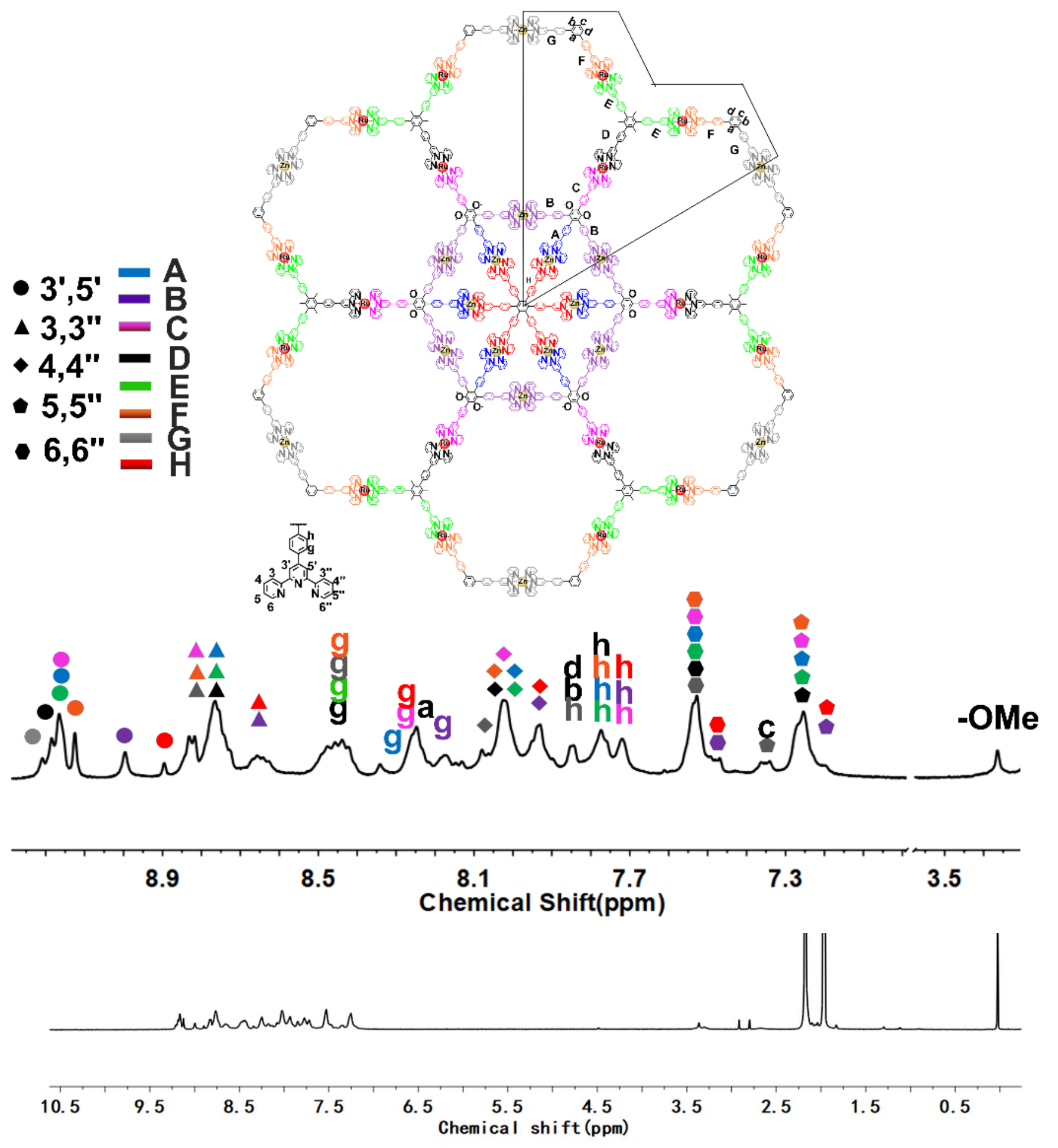

Figure S48: ${ }^{1} \mathrm{H}$ NMR full spectrum $(500 \mathrm{MHz})$ of $\mathbf{W} \mathbf{1}$ in $\mathrm{CD}_{3} \mathrm{CN}$ (min 10.5-0.0 ppm). 


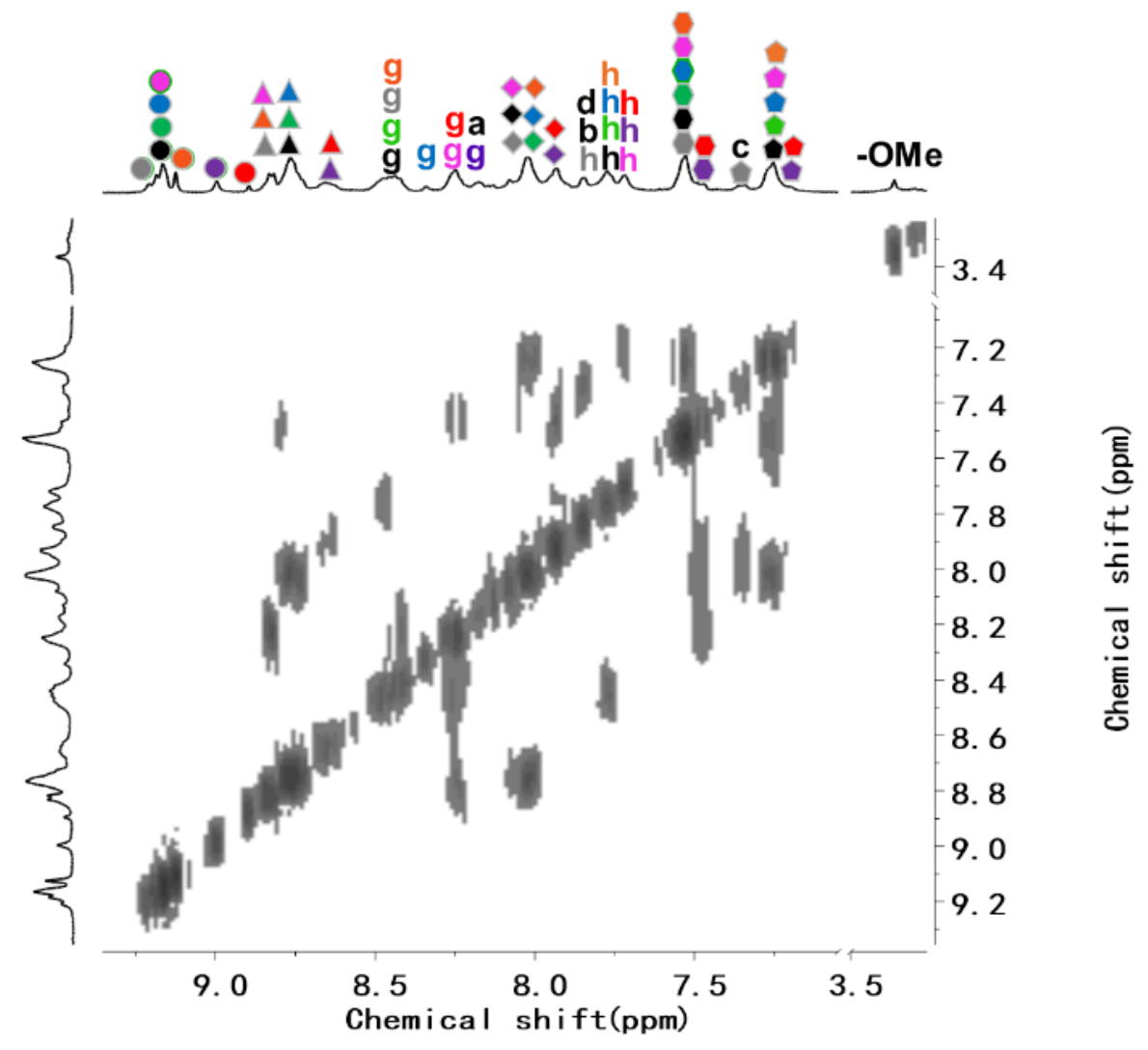

Figure S49: 2D COSY spectrum (500 MHz) of $\mathbf{W 1}$ in $\mathrm{CD}_{3} \mathrm{CN}$.

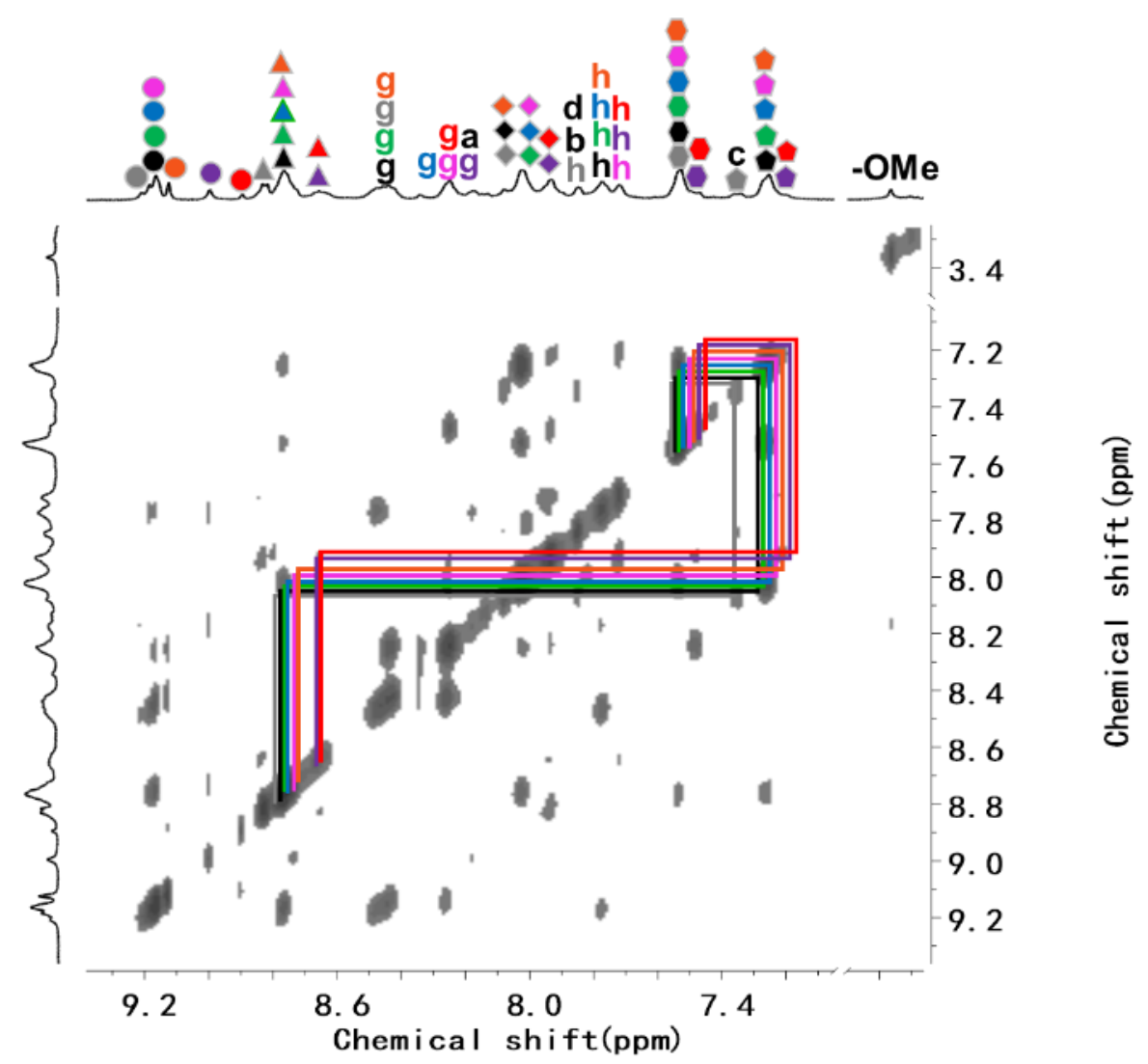

Figure S50: 2D NOESY spectrum $(500 \mathrm{MHz})$ of $\mathbf{W} \mathbf{1}$ in $\mathrm{CD}_{3} \mathrm{CN}$. 


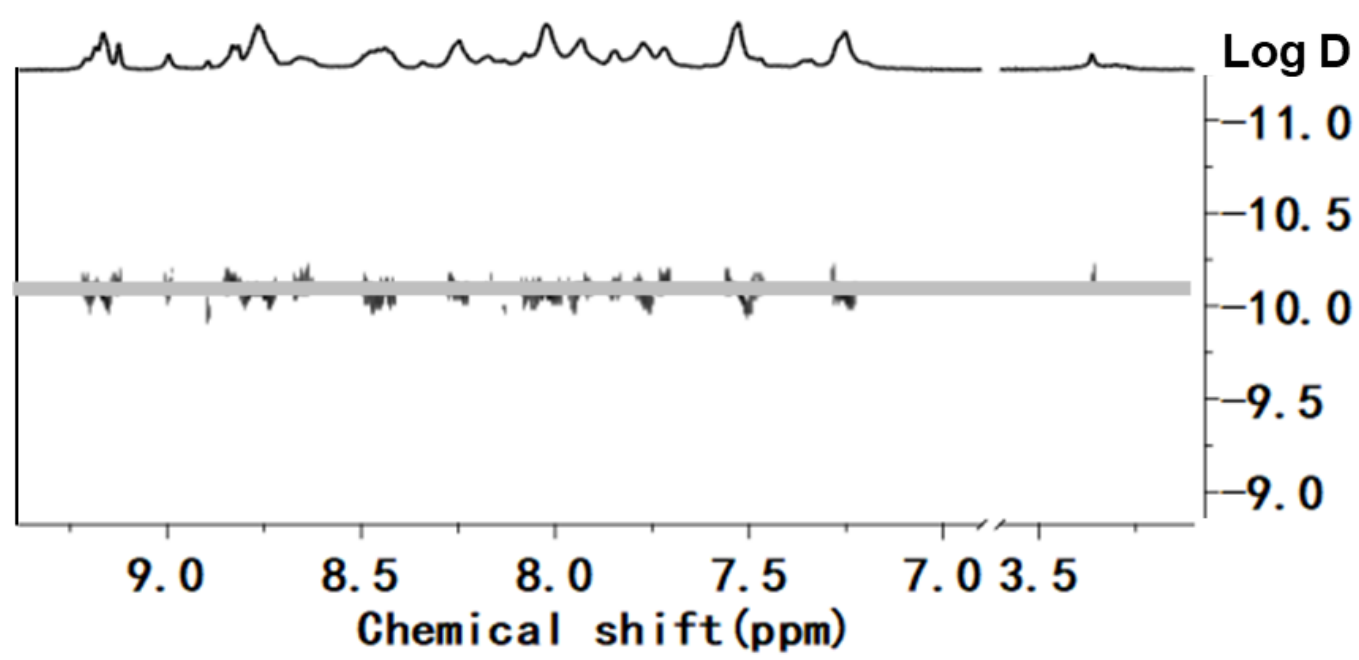

Figure S51: 2D DOSY NMR spectra of W1.

The radius ( $r$ ) of the hexagon-fused wheel macromolecules $\mathbf{W} \mathbf{1}$ was roughly estimated using a modified Stokes-Einstein equation: ${ }^{\mathrm{S} 5}$

$D=\frac{\mathrm{kT}}{6 \pi \eta \mathrm{Rf}}$

take $\mathrm{f} \approx 1.2$

$\mathrm{D}=8.31 \times 10^{-11} \mathrm{~m}^{2} \mathrm{~s}^{-1}$

$\mathrm{k}=1.38 \times 10^{-23} \mathrm{~N} \mathrm{~m} \mathrm{~K}^{-1}$

$\mathrm{T}=298 \mathrm{~K}$

$\mu=3.7 \times 10^{-4} \mathrm{~N} \mathrm{~m}^{-2} \mathrm{~s}\left(\mathrm{CD}_{3} \mathrm{CN}\right)$

$R=\frac{\mathrm{kT}}{6 \pi \mu \mathrm{Df}} \approx 5.9 \times 10^{-9} \mathrm{~m} \approx 5.9 \mathrm{~nm}$

The radius of the $\mathrm{W} 1$ was determined to be $5.9 \mathrm{~nm}$, which is smaller than the computer modeling possibly caused by the broad NMR signal. 


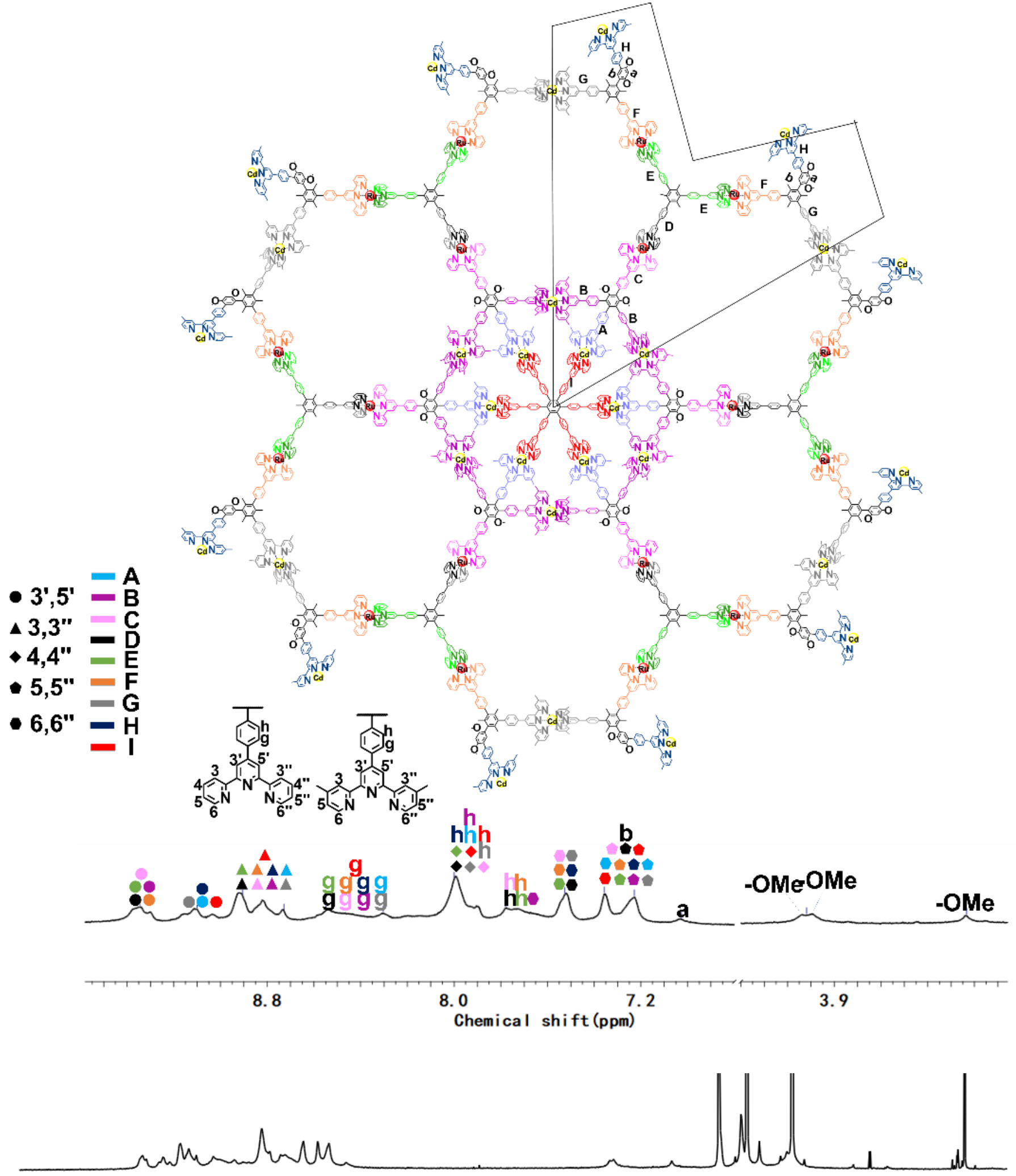

\begin{tabular}{llllllllll|l|l|}
\hline 10.5 & 9.5 & 8.5 & 7.5 & 6.5 & 5.5 & 4.5 & 3.5 & 2.5 & 1.5 & 0.5 &
\end{tabular}

Figure S52: ${ }^{1} \mathrm{H}$ NMR full spectrum (500 MHz) of $\mathbf{P 1} \mathrm{CD}_{3} \mathrm{CN} / \mathrm{DMSO}(2 / 1)$ (min 10.5-0.0 ppm). 


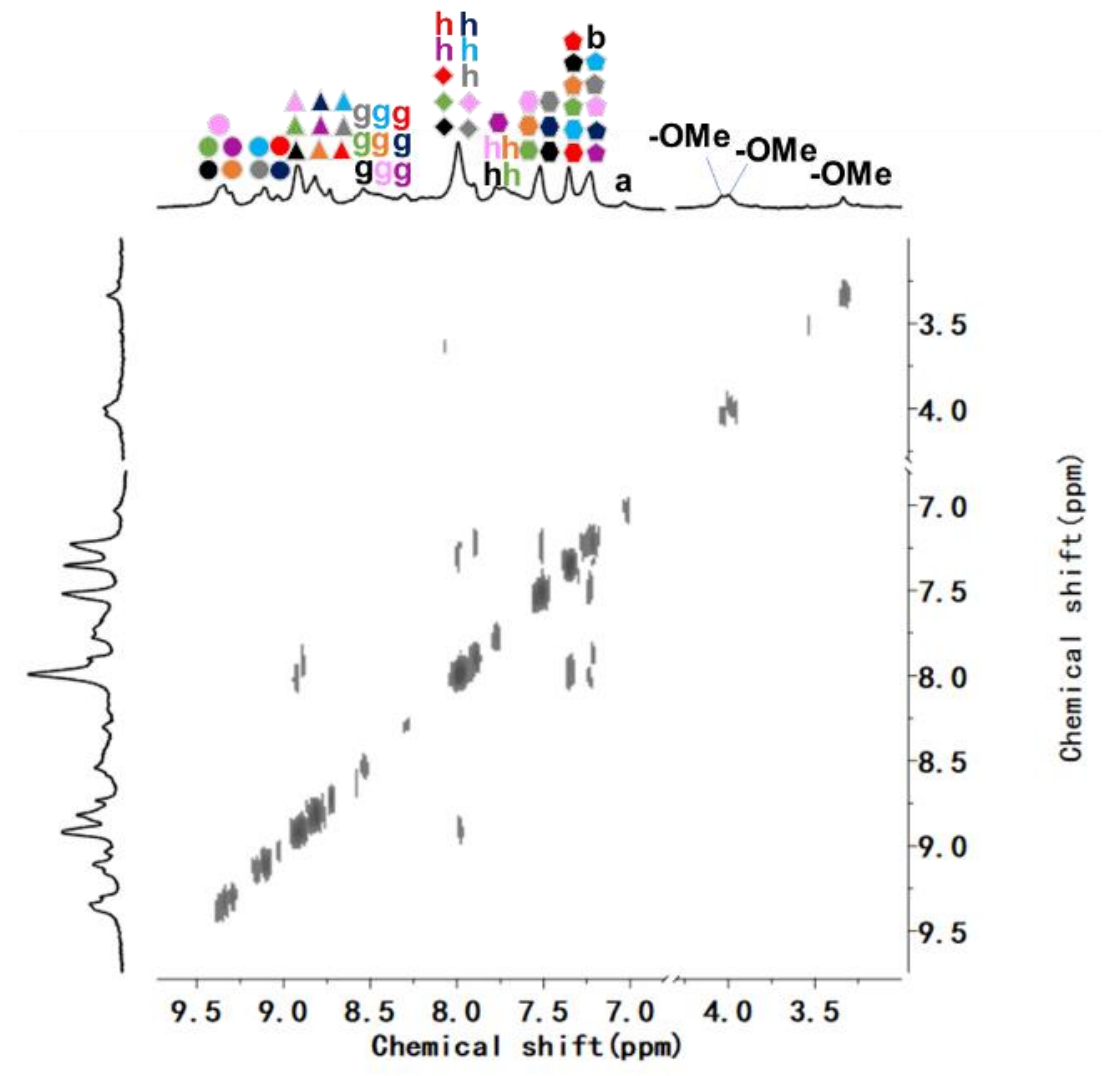

Figure S53: 2D COSY spectrum (500 MHz) of $\mathbf{P 1}$ in $\mathrm{CD}_{3} \mathrm{CN} / \mathrm{DMSO}(2 / 1)$.

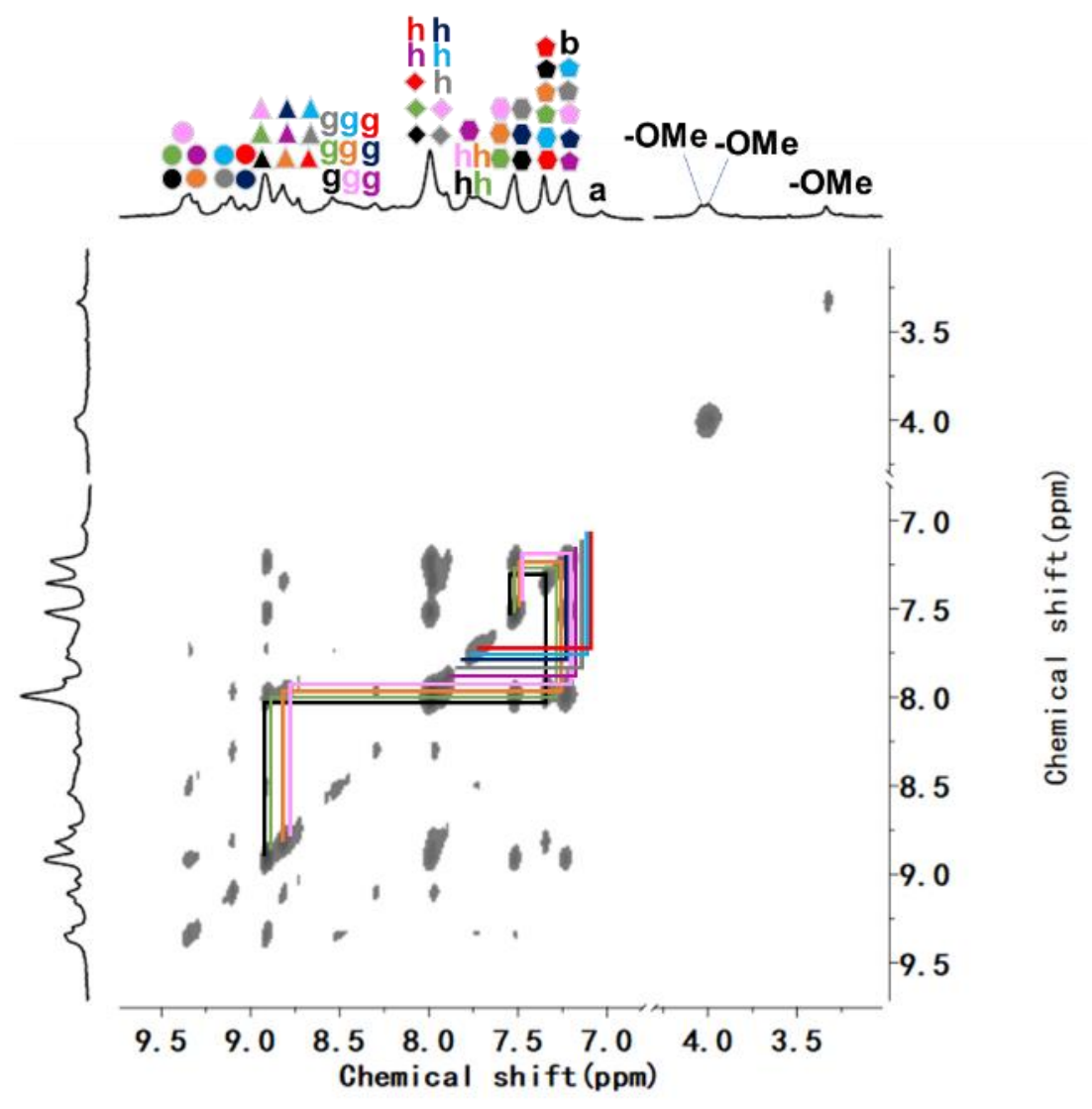

Figure S54: 2D NOESY spectrum (500 MHz) of $\mathbf{P 1}$ in $\mathrm{CD}_{3} \mathrm{CN} / \mathrm{DMSO}(2 / 1)$. 


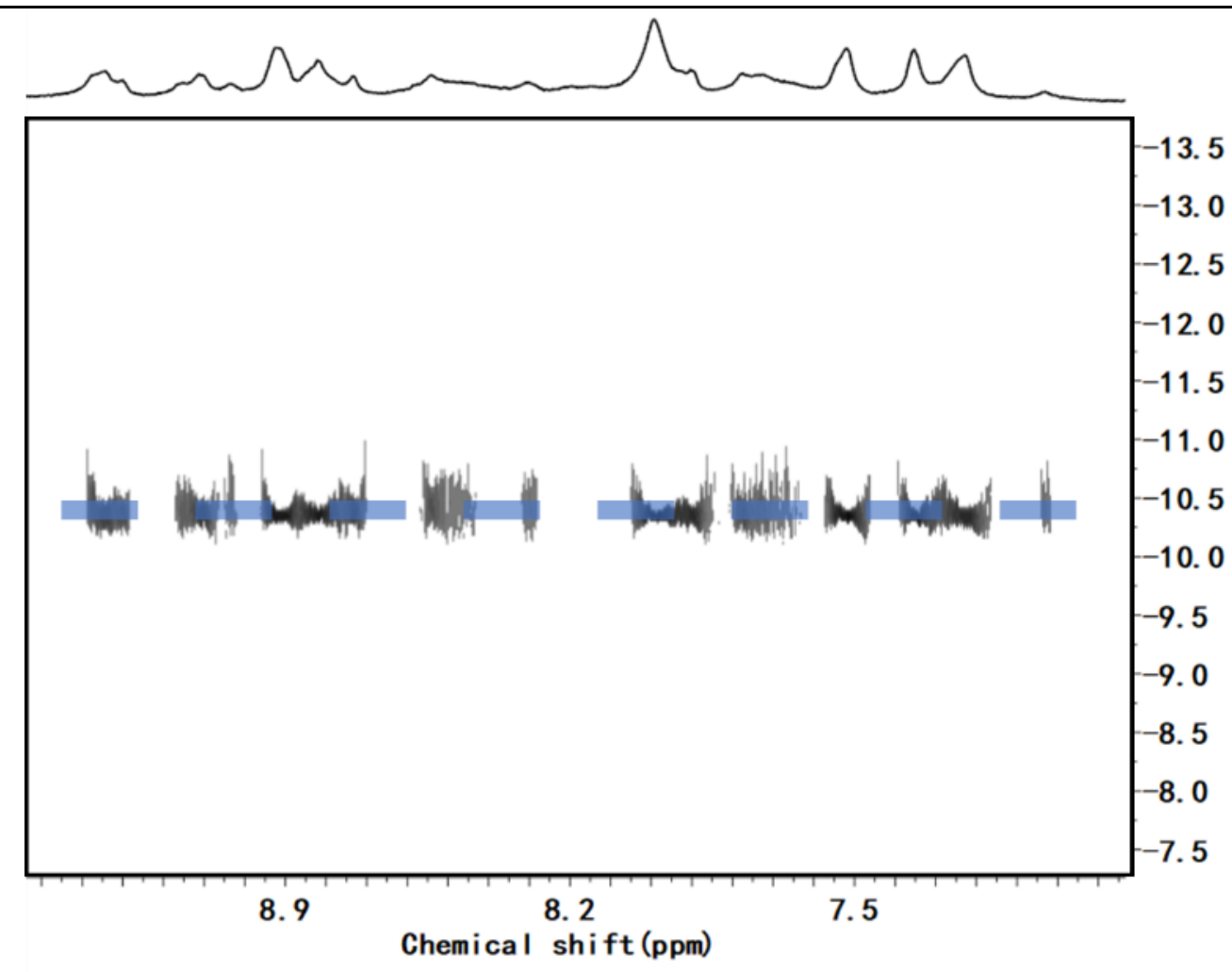

Figure S55: 2D DOSY NMR spectra of P1.

The radius (r) of the prism-like architecture P1 was roughly estimated using the Stokes-Einstein equation:

$D=\frac{\mathrm{kT}}{6 \pi \eta \mathrm{R}}$

$\mathrm{D}=4.26 \times 10^{-11} \mathrm{~m}^{2} \mathrm{~s}^{-1}$

$\mathrm{k}=1.38 \times 10^{-23} \mathrm{~N} \mathrm{~m} \mathrm{~K}^{-1}$

$\mathrm{T}=298 \mathrm{~K}$

$\mu \approx 7.0 \times 10^{-4} \mathrm{~N} \mathrm{~m}^{-2} \mathrm{~s}\left(\mathrm{DMSO}-\mathrm{d}^{5} / \mathrm{CD}_{3} \mathrm{CN}(\mathrm{v} / \mathrm{v}, 1: 2)\right)^{\mathrm{s} 6}$

$R=\frac{\mathrm{kT}}{6 \pi \mu \mathrm{Df}} \approx 7.3 \times 10^{-9} \mathrm{~m}=7.3 \mathrm{~nm}$

The radius of the $\mathbf{P 1}$ was determined to be $7.3 \mathrm{~nm}$, which is consistent with the results of computer modeling.

\section{ESI-MS spectra for ligands and complexes.}




\section{Experimental}

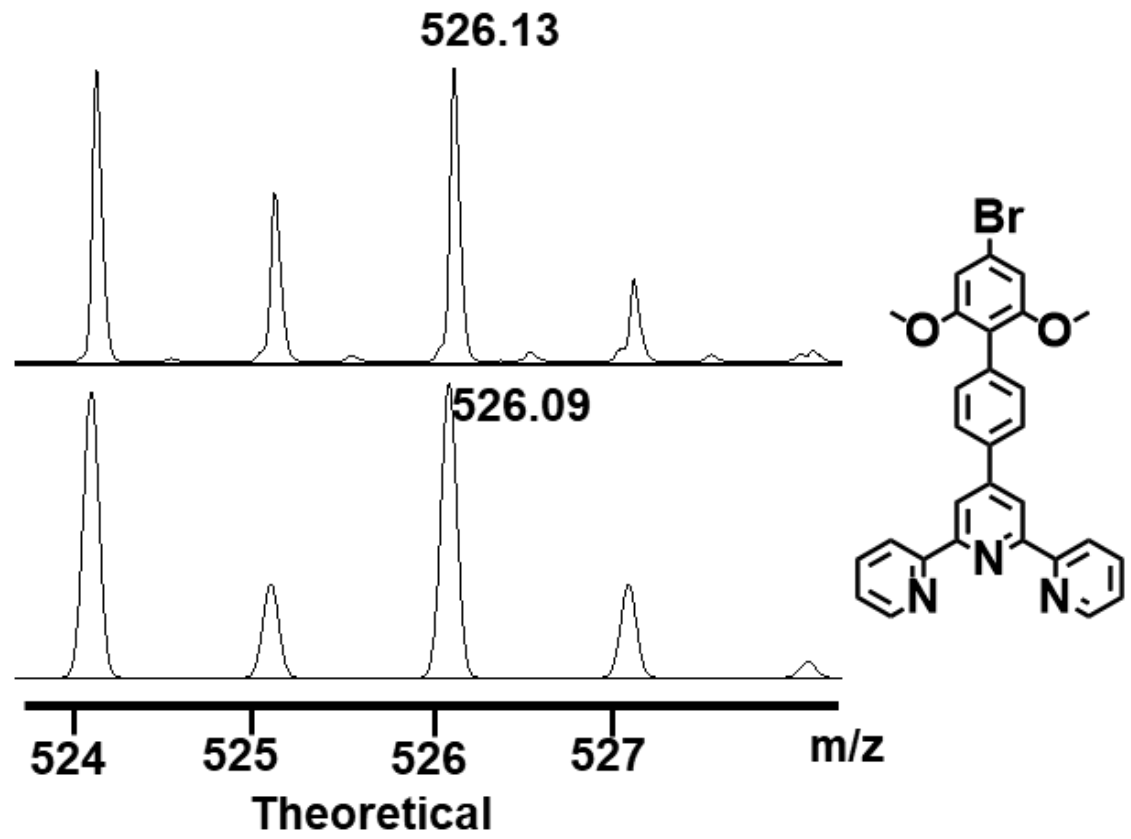

Figure S56: ESI-MS spectrum of Compound S4.

Experimental

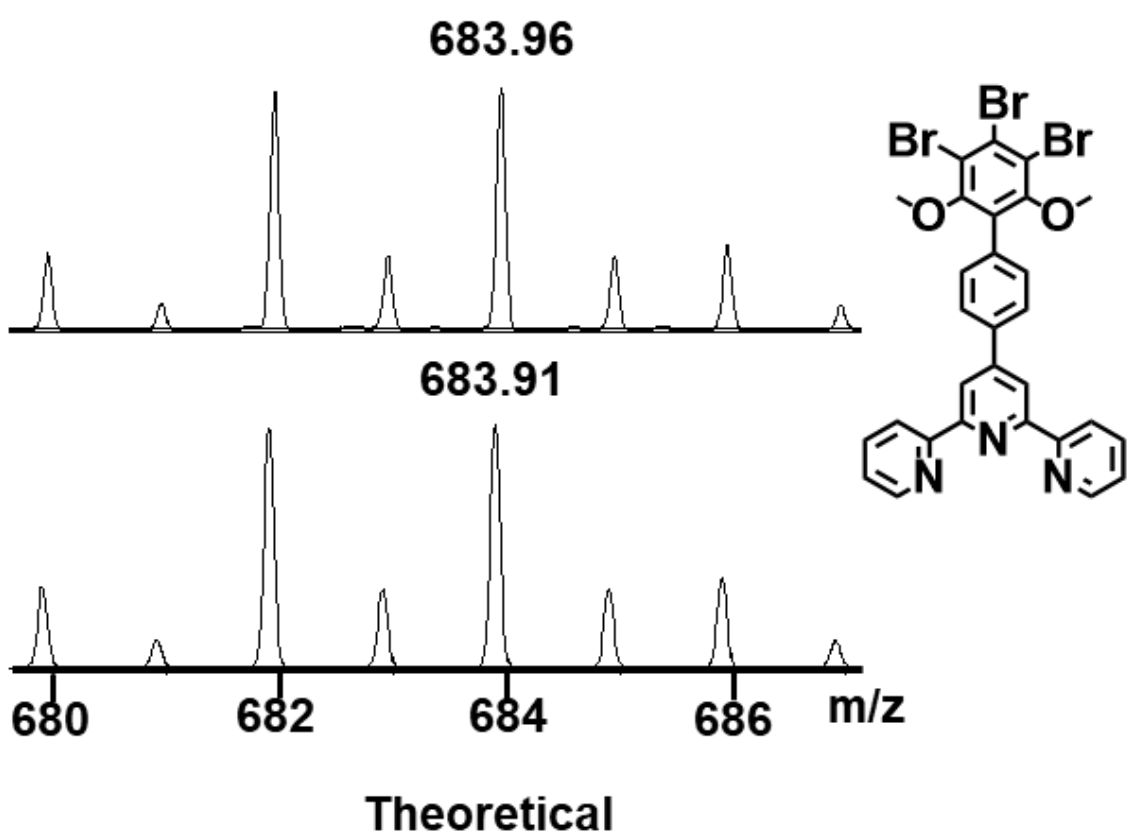

Figure S57: ESI-MS spectrum of Compound S5. 


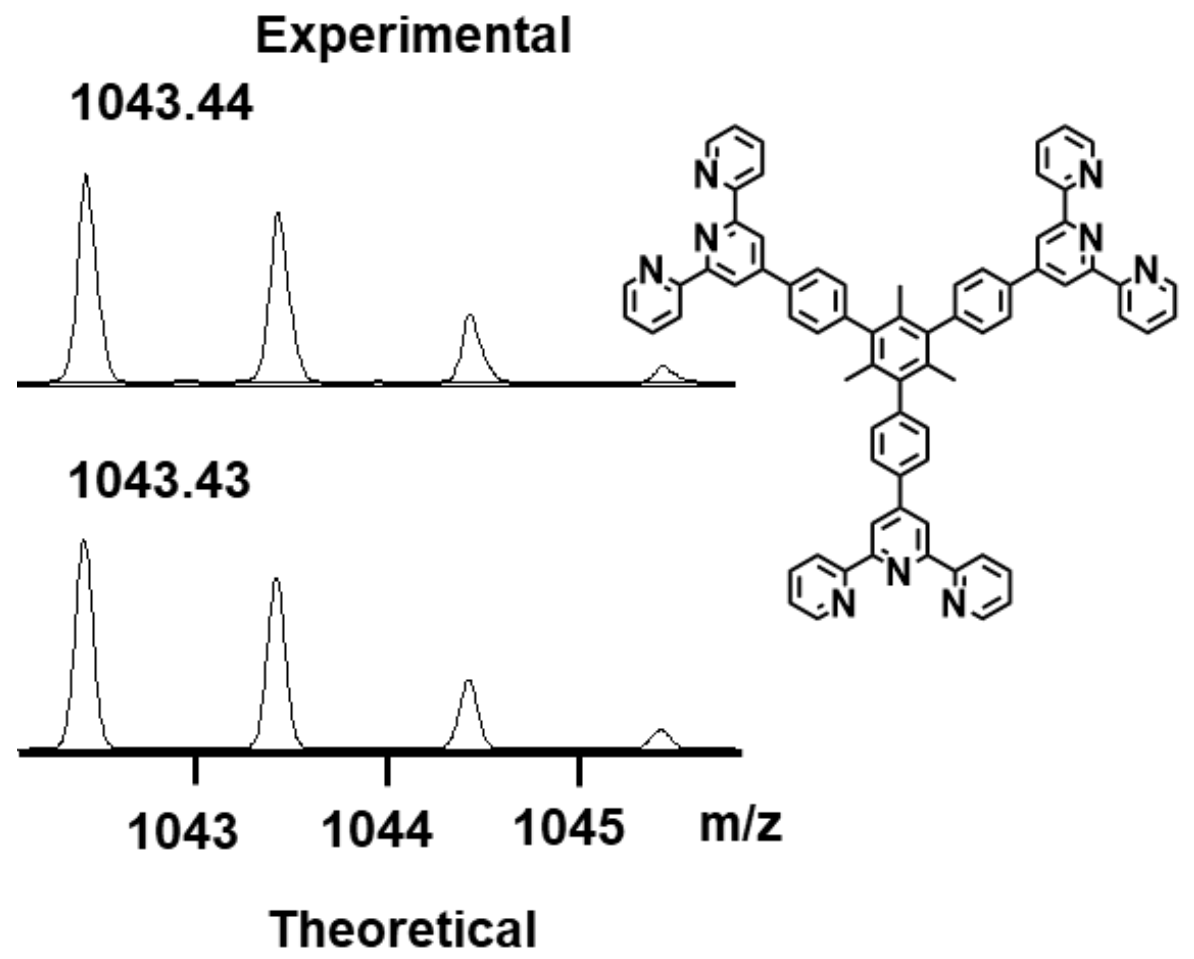

Figure S58: ESI-MS spectrum of Compound 2.

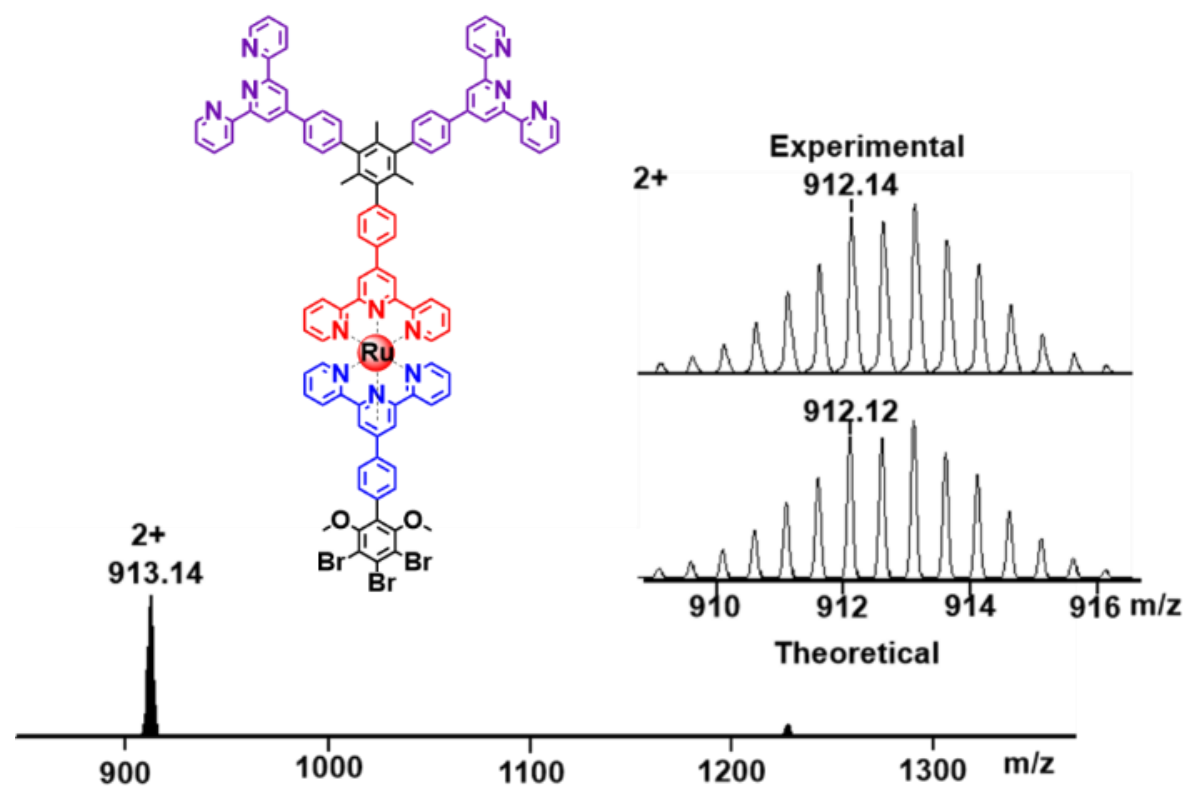

Figure S59: ESI-MS spectrum of Complex $3\left(\mathrm{Cl}^{-}\right.$as counterion). 


\section{Experimental}

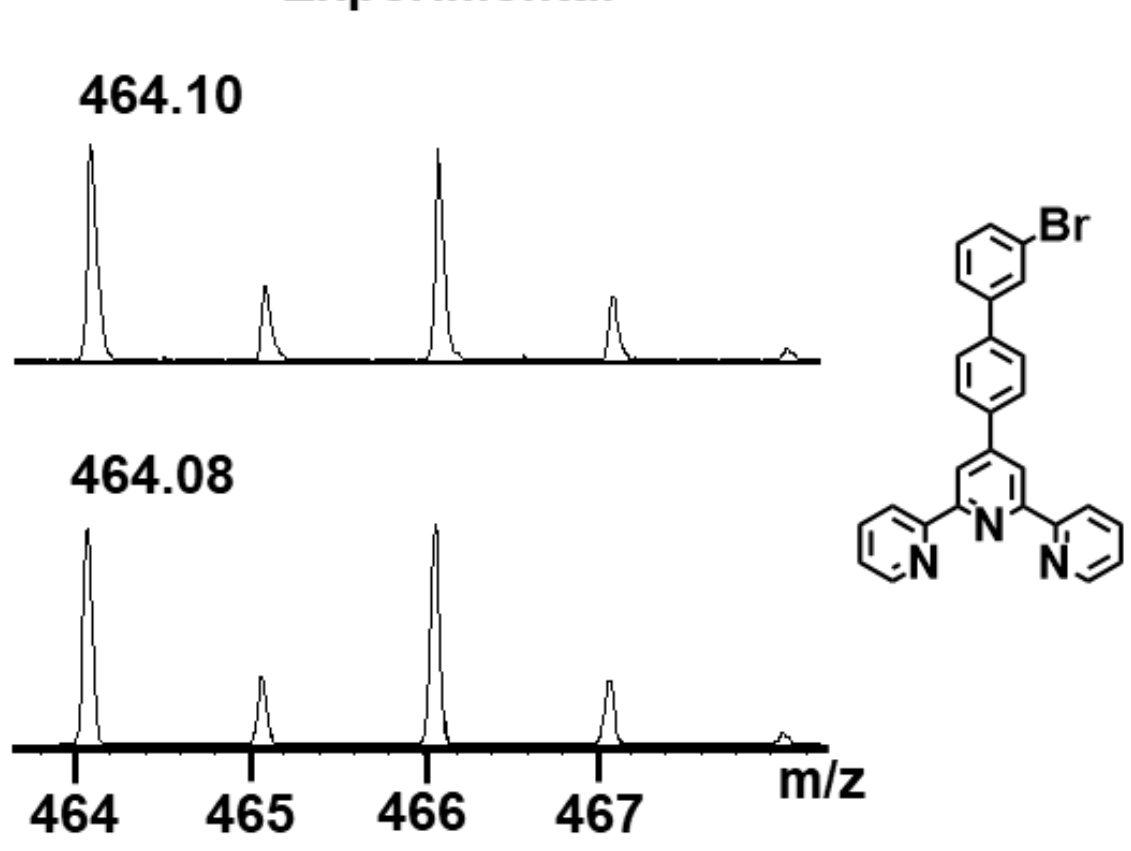

Theoretical

Figure S60: ESI-MS spectrum of Compound S8.

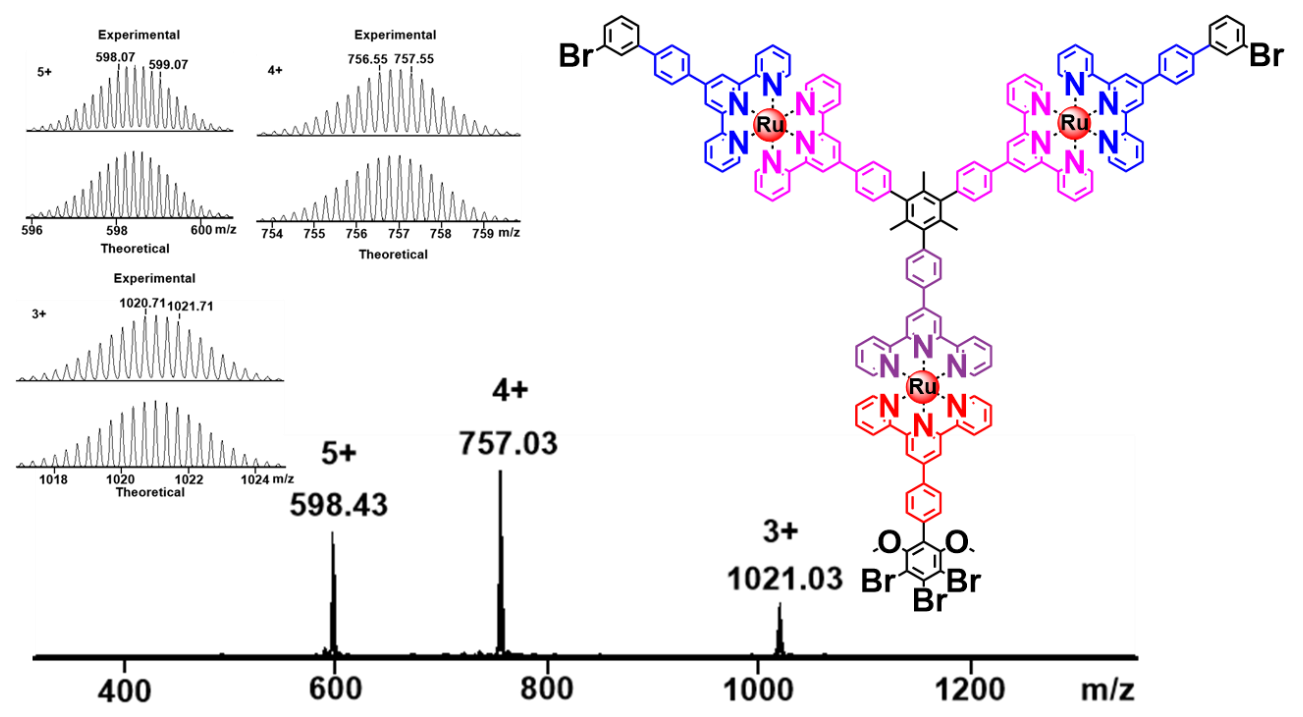

Figure S61: ESI-MS spectrum of Complex $5\left(\mathrm{Cl}^{-}\right.$as counterion). 


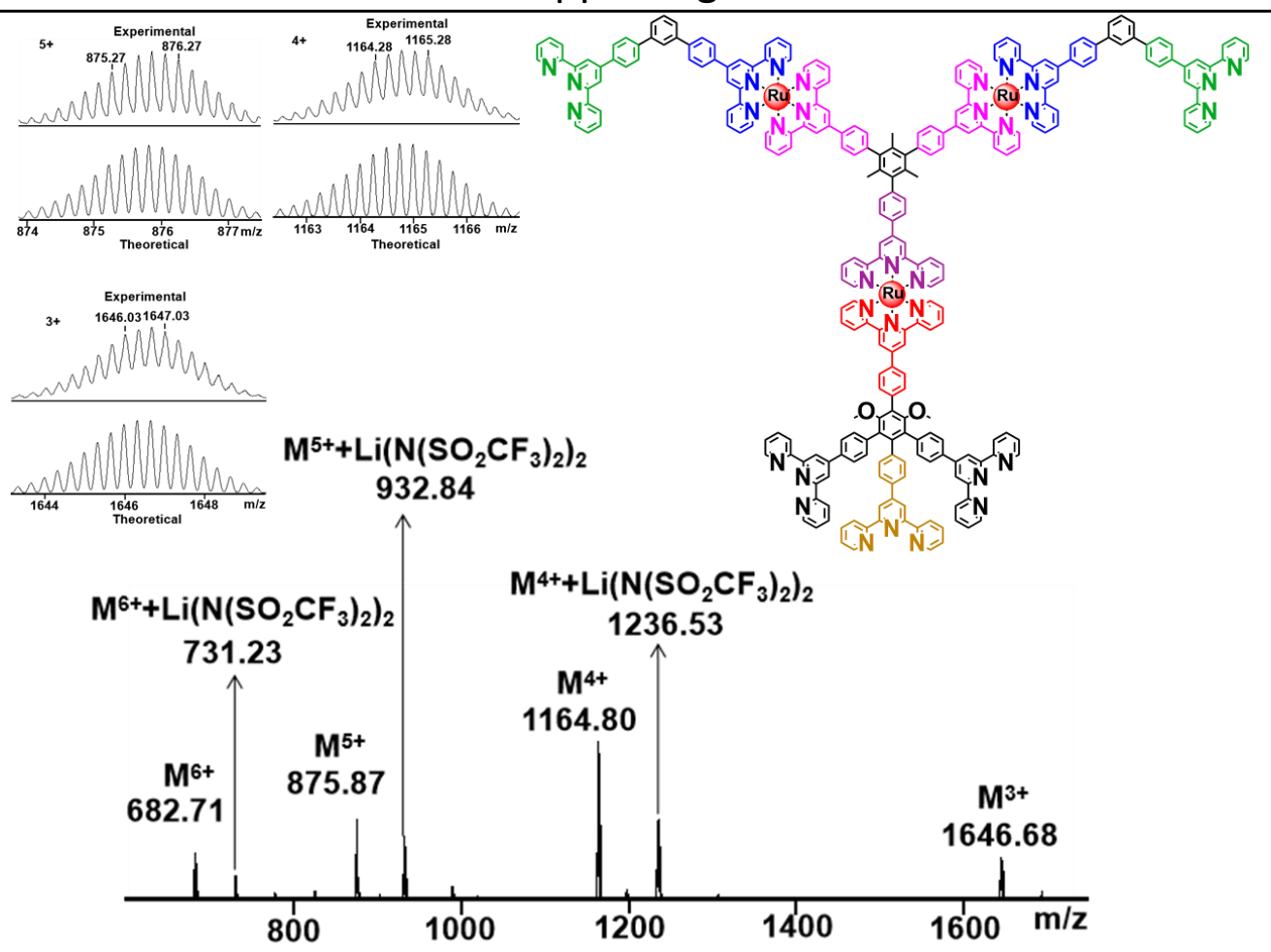

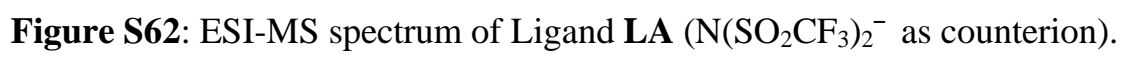

\section{Experimental}

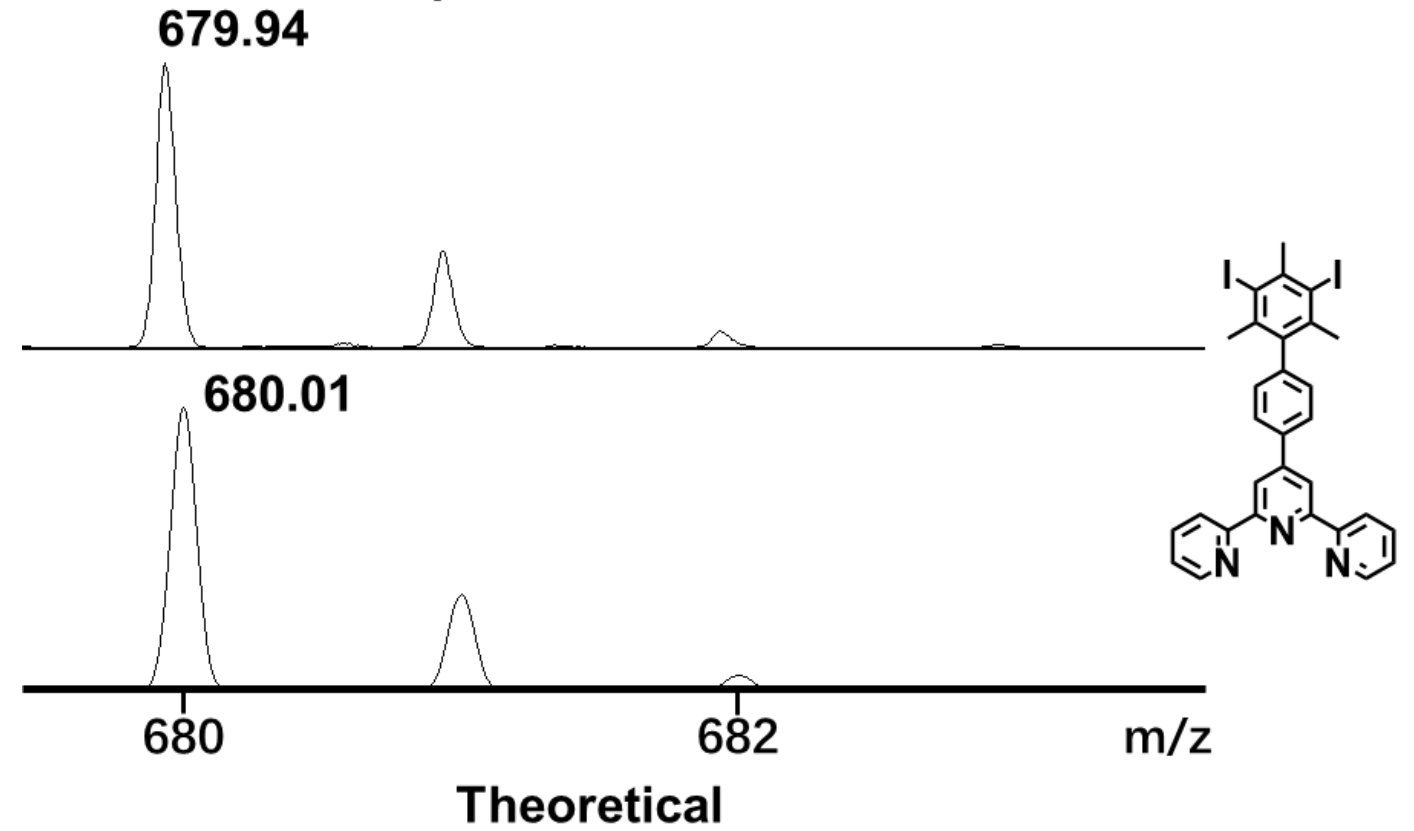

Figure S63: ESI-MS spectrum of Compound S9. 


\section{Experimental}

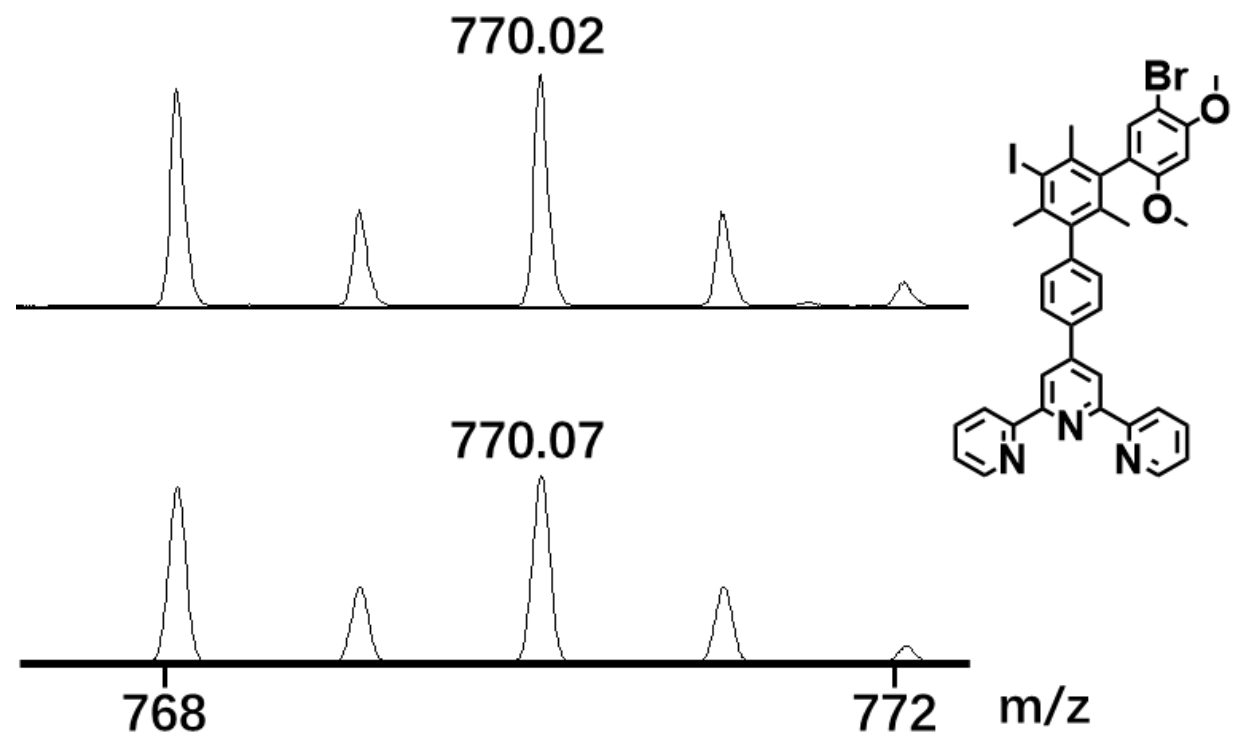

Theoretical

Figure S64: ESI-MS spectrum of Compound S11.

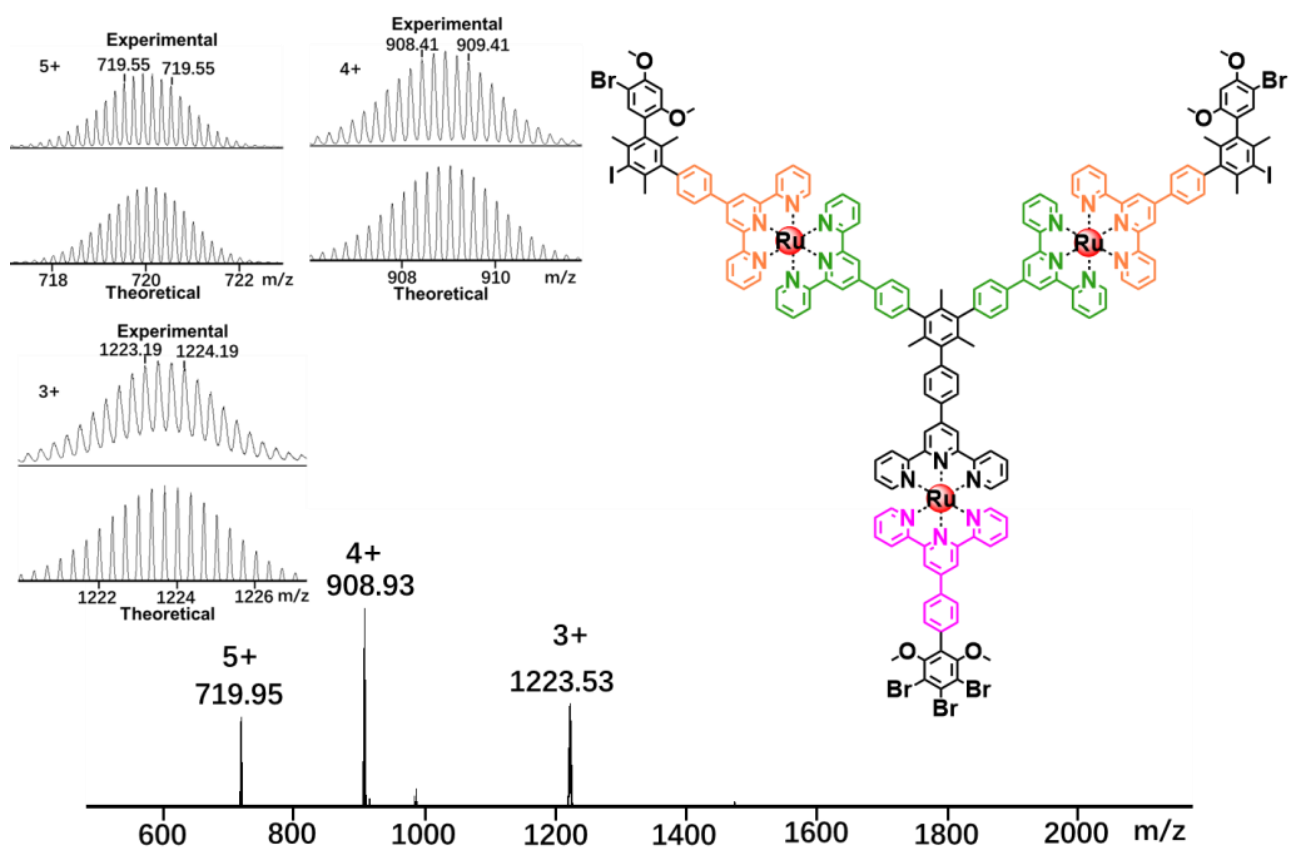

Figure S65: ESI-MS spectrum of Complex $8\left(\mathrm{Cl}^{-}\right.$as counterion). 


\section{Experimental}

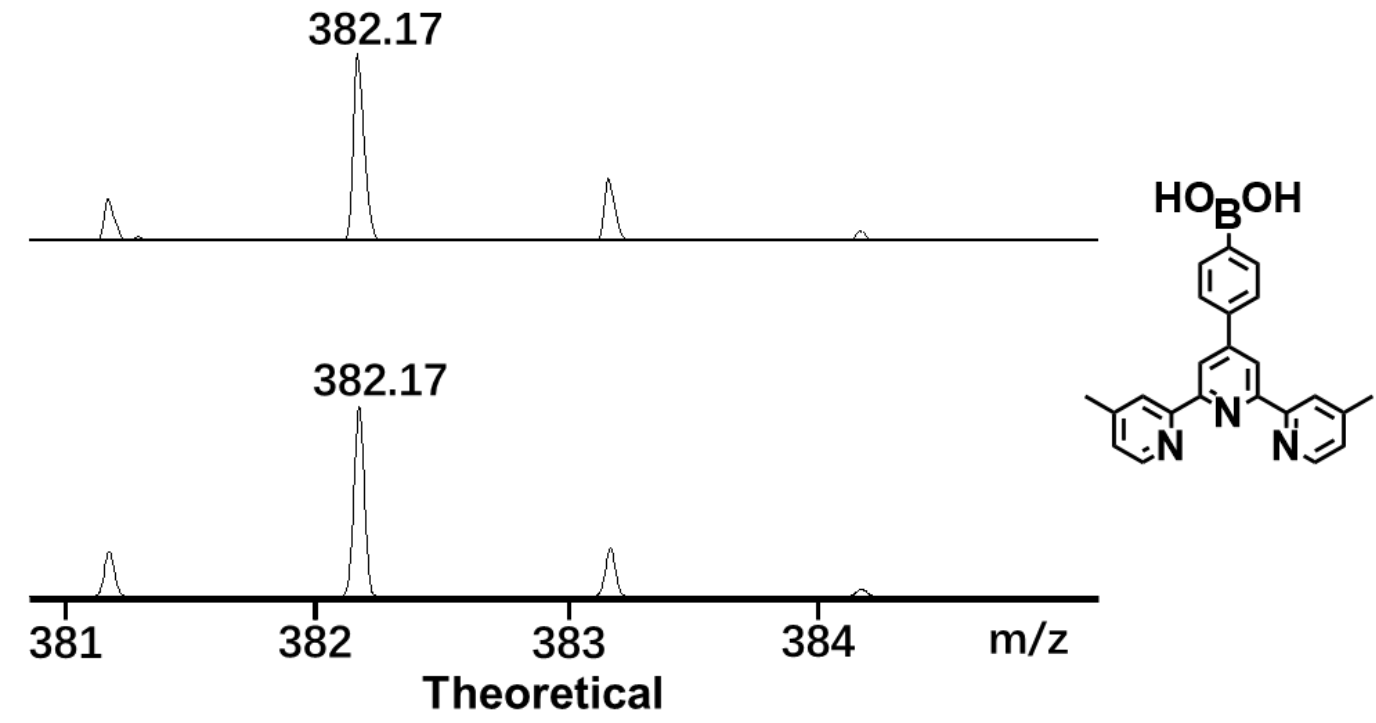

Figure S66: ESI-MS spectrum of Compound 9.
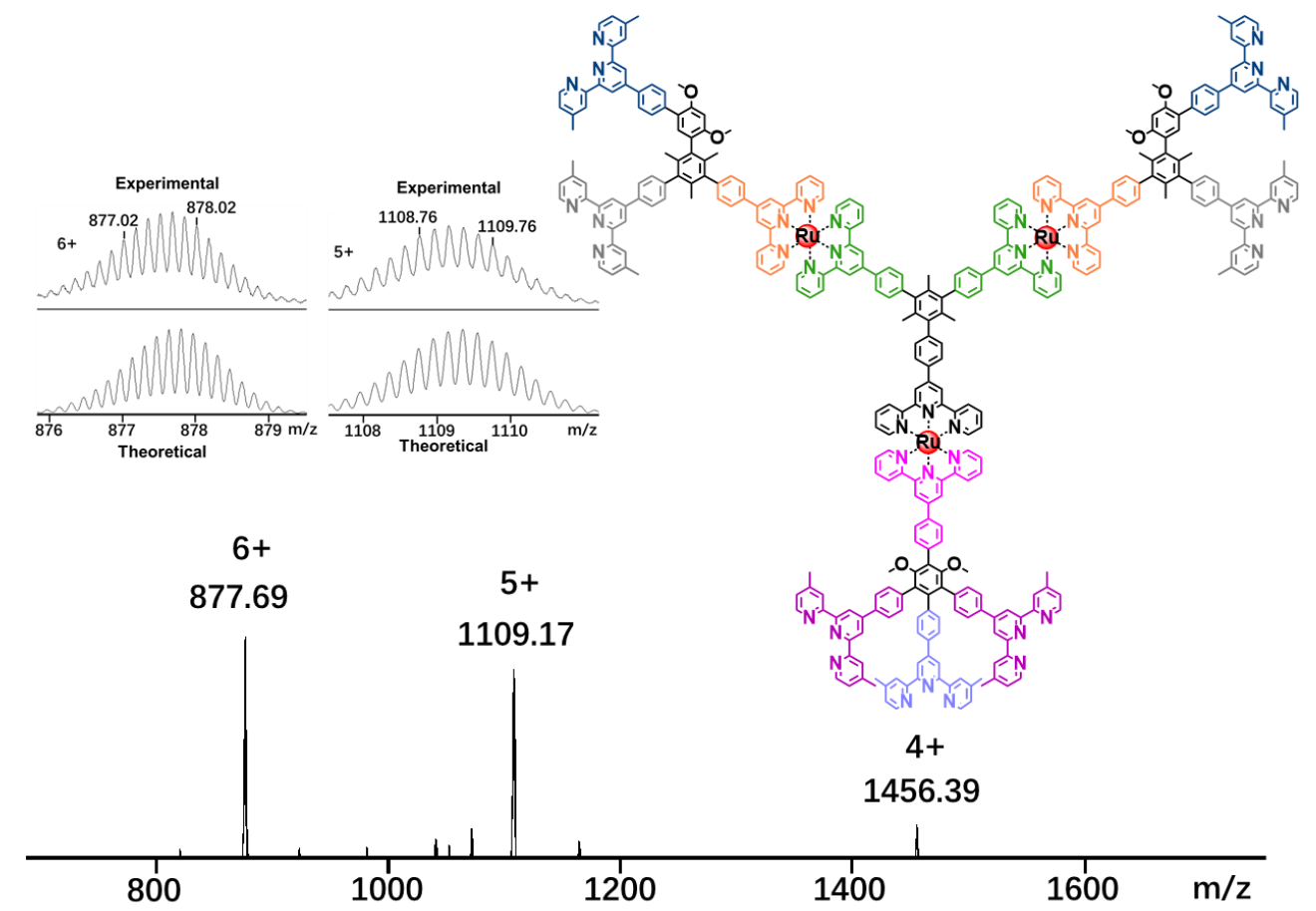

Figure S67: ESI-MS spectrum of Ligand $\mathbf{L B}\left(\mathrm{N}\left(\mathrm{SO}_{2} \mathrm{CF}_{3}\right)_{2}{ }^{-}\right.$as counterion). 


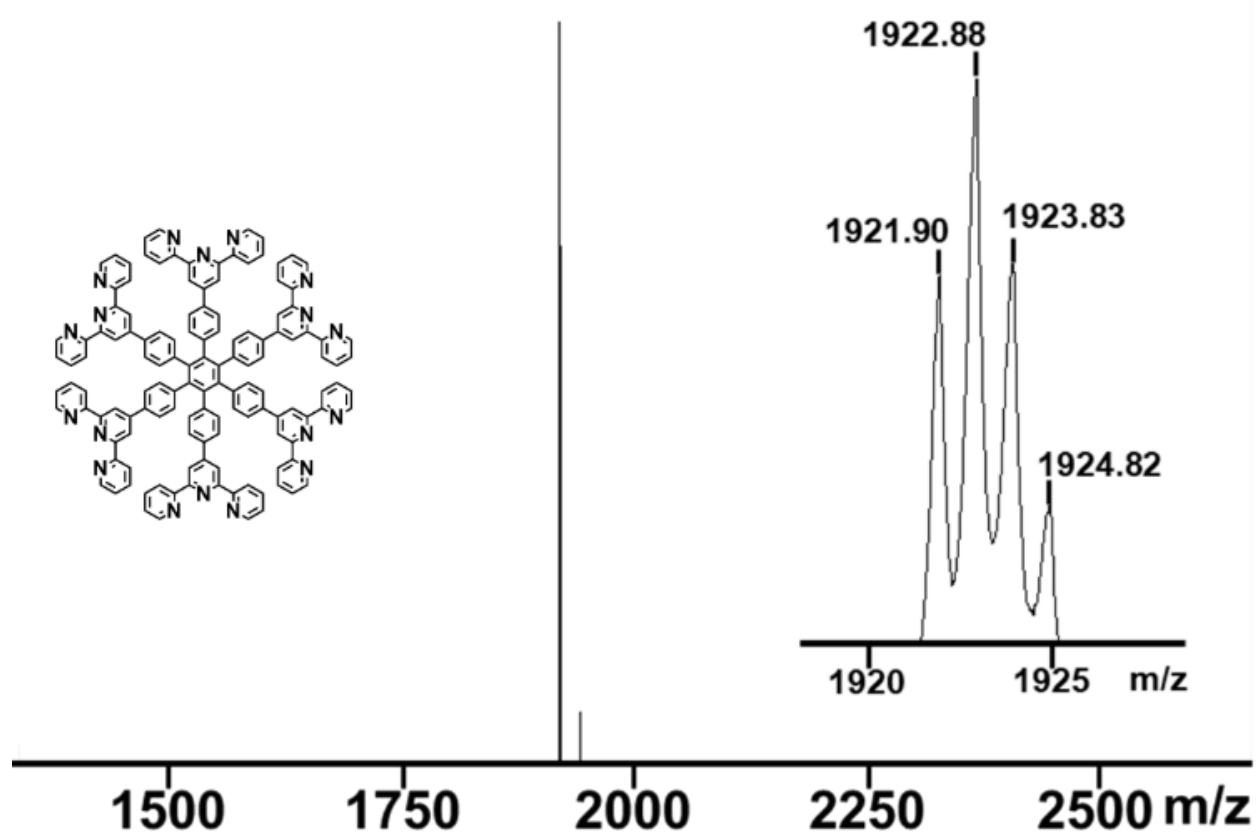

Figure S68: MALDI-TOF mass spectrum of Ligand $\mathbf{L H}$.

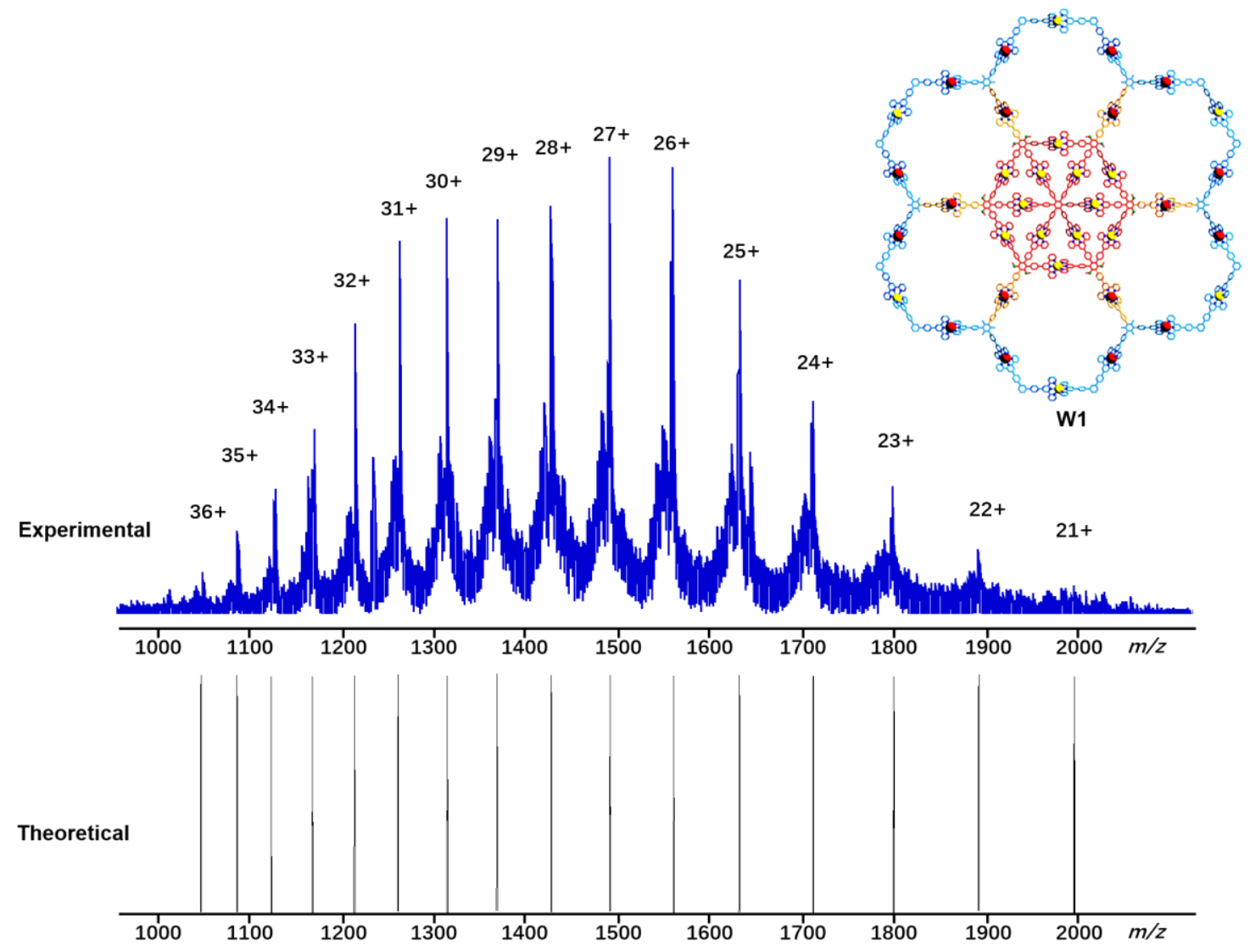

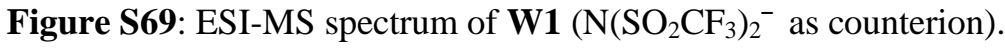




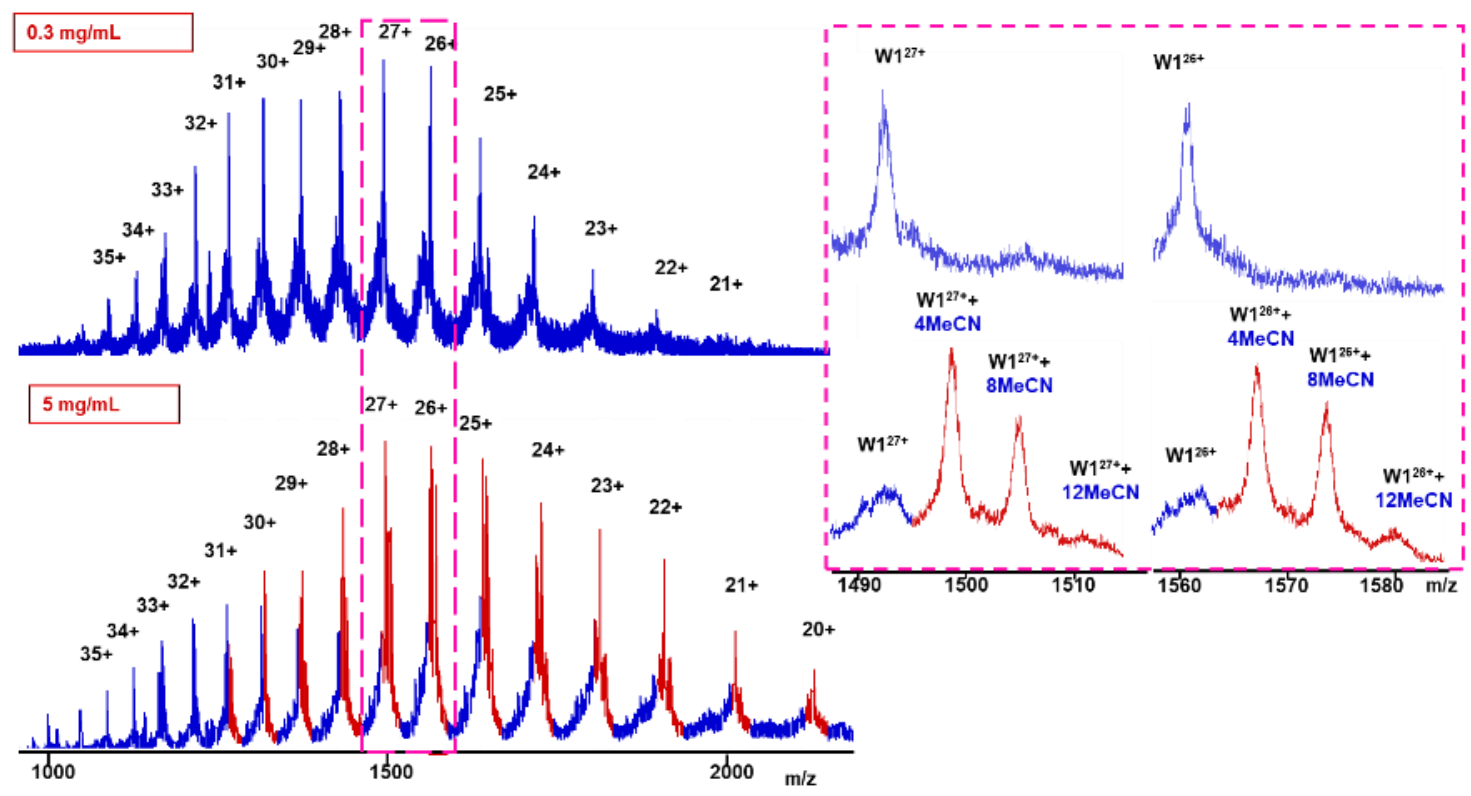

Figure S70: The ESI-MS spectrum of $\mathbf{W} 1$ at $0.3 \mathrm{mg} \mathrm{mL}^{-1}$ and $5 \mathrm{mg} \mathrm{mL}^{-1}$ in $\mathrm{CH}_{3} \mathrm{CN}$, two ESI-MS charge states of W1 as representative peaks for absorption solvent $\mathrm{CH}_{3} \mathrm{CN}$.

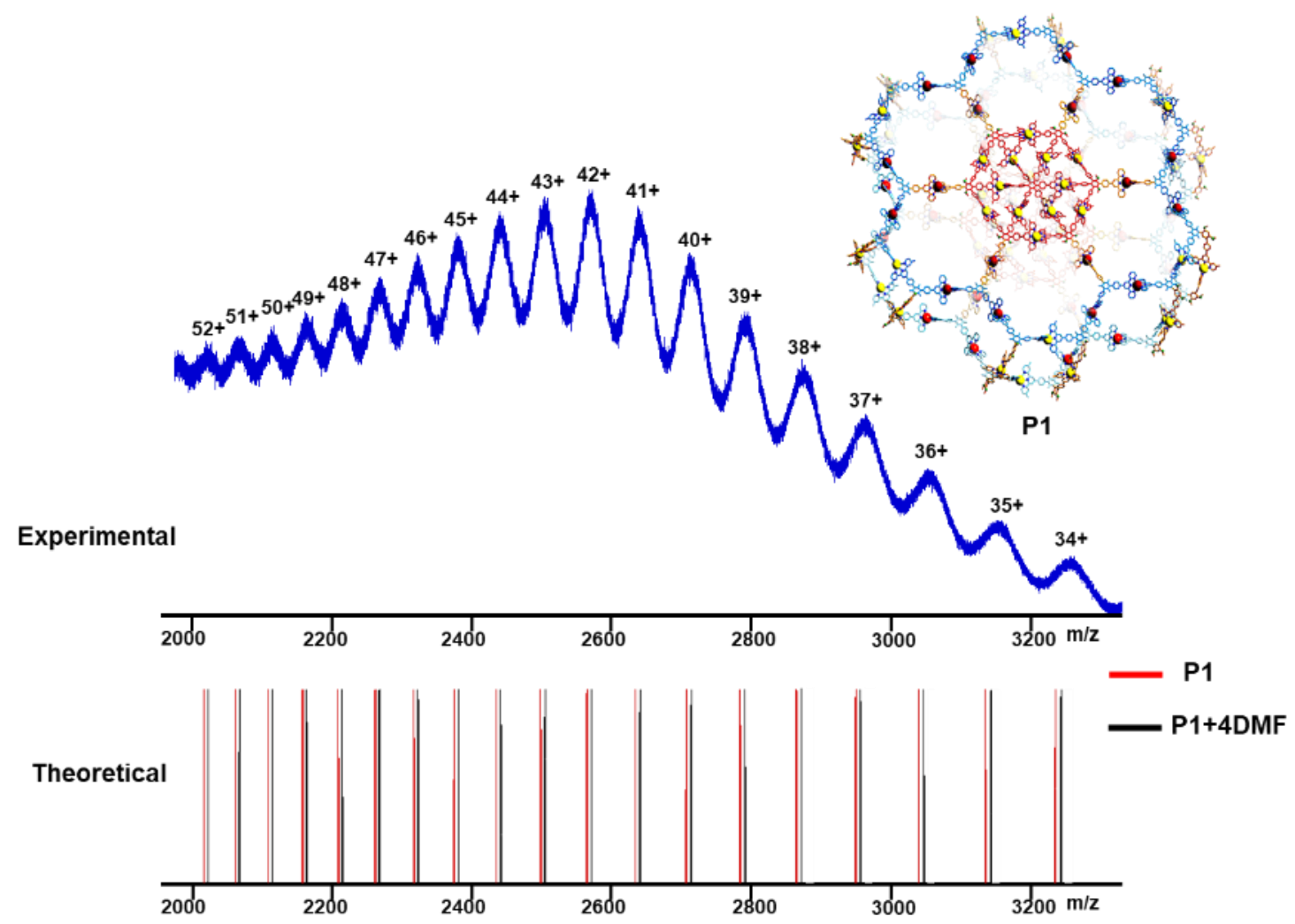

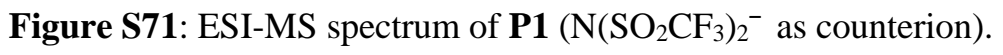

\section{TEM images and Molecular models.}


Electronic Supporting Information

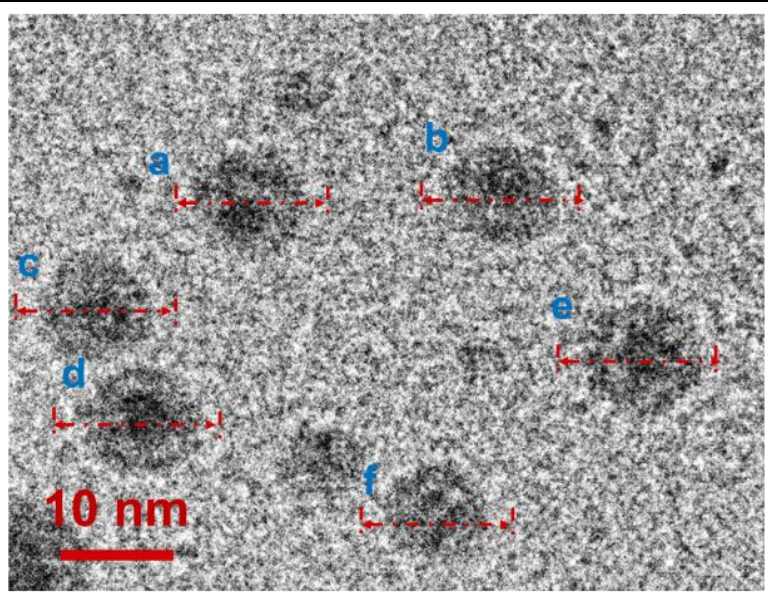

\begin{tabular}{|c|c|}
\hline Entry & Size $(\mathrm{nm})$ \\
\hline a & 13.6 \\
\hline b & 13.8 \\
\hline c & 13.7 \\
\hline$d$ & 13.8 \\
\hline e & 13.9 \\
\hline f & 13.6 \\
\hline
\end{tabular}

Figure S72: TEM image and the size of hexagon-fused wheel W1.

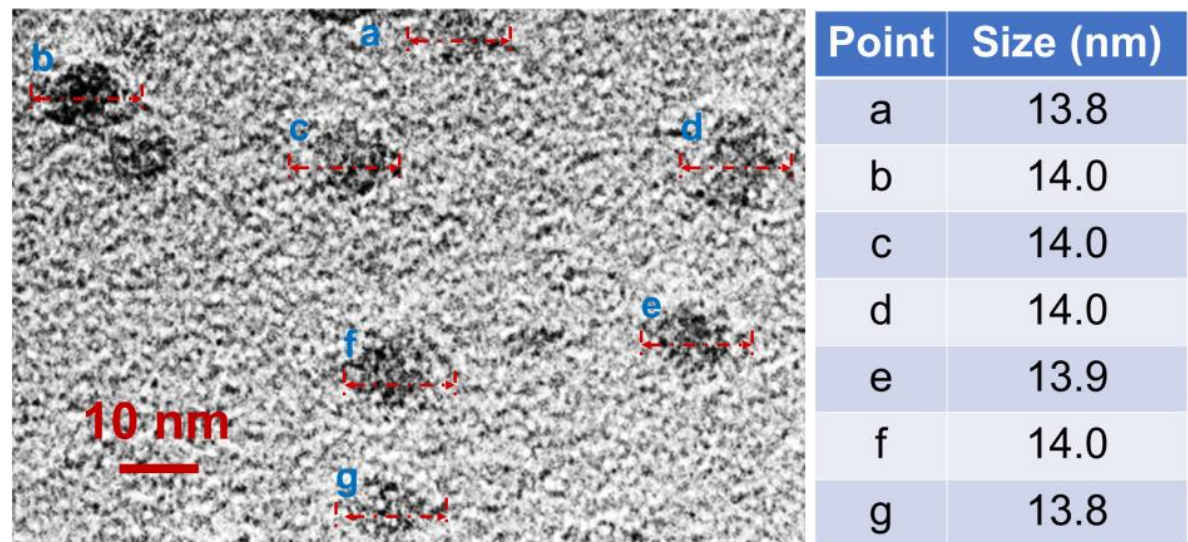

Figure S73: TEM image and the size of double-decker dodecagonal prism-like architecture P1.

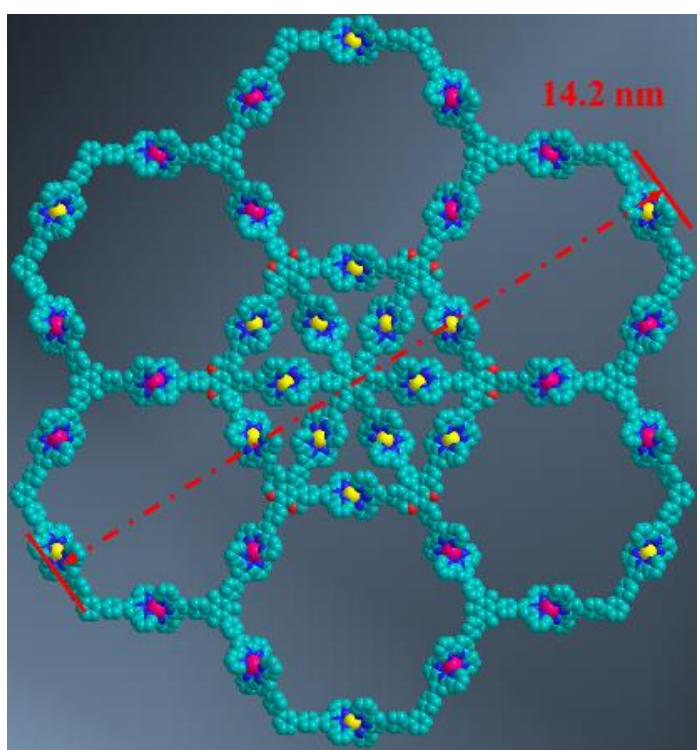

Figure S74: Energy-minimized structure of W1. 

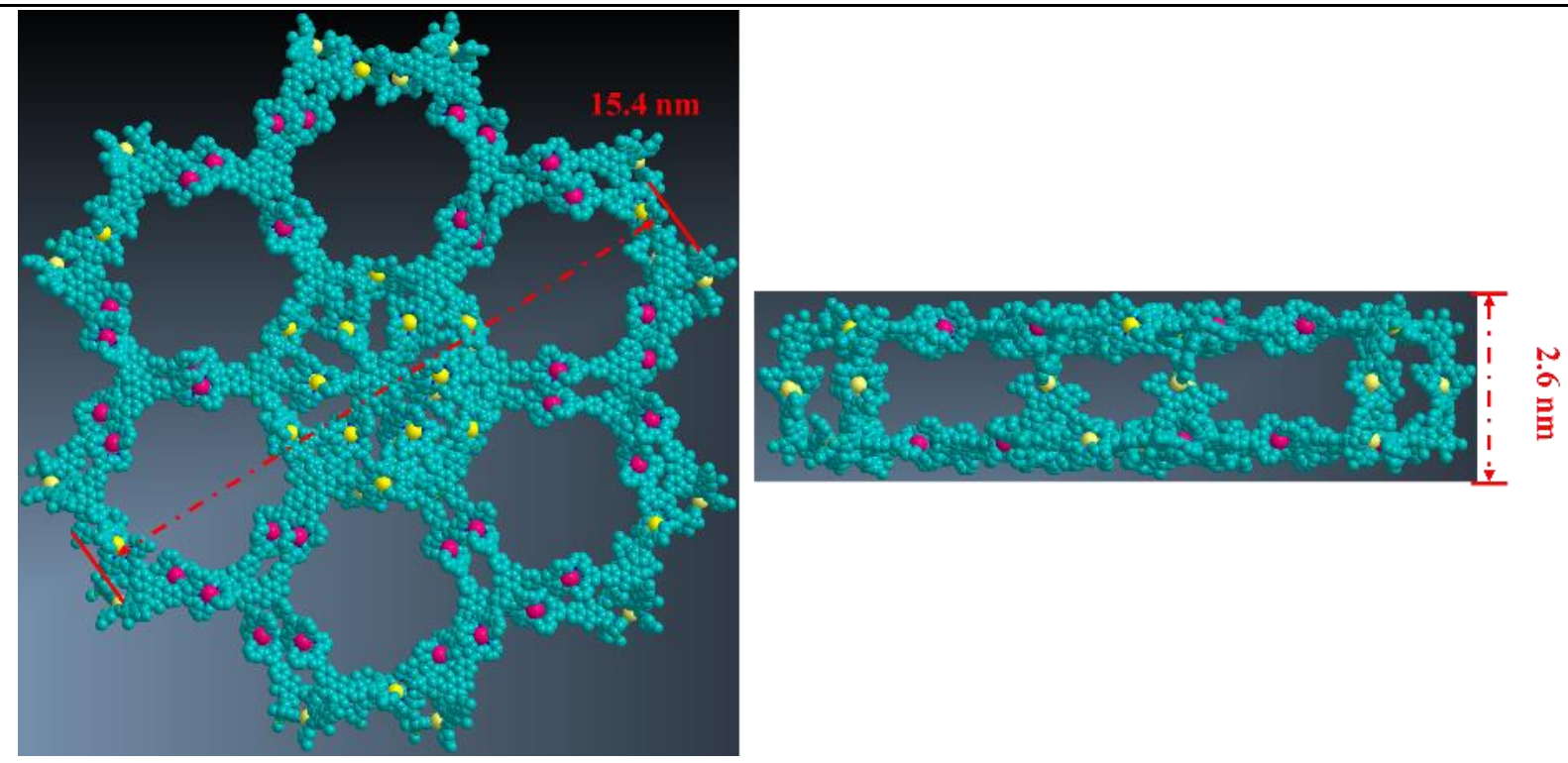

Figure S75: Energy-minimized structure of $\mathbf{P 1}$.

\section{Supplementary References}

S1. Wang, J.-L.; Li, X.; Lu,X. C.; Hsieh, I-F.; Cao, Y.; Moorefield, C. N.; Wesdemiotis, C.; Cheng, S. Z. D.; Newkome, G. R., Stoichiometric self-assembly of shape-persistent 2D complexes: a facile route to a symmetric supramacromolecular spoked wheel. J. Am. Chem. Soc. 2011, 133, 11450-11453.

S2. Jarosz, P.; Lotito, K.; Schneider, J.; Kumaresan, D.; Schmehl, R.; Eisenberg, R., Platinum(II) Terpyridyl-Acetylide Dyads and Triads with Nitrophenyl Acceptors via a Convenient Synthesis of a Boronated Phenylterpyridine. Inorg. Chem. 2009, 48, 2420-2428.

S3. Samanta, S.; Beharry, A. A.; Sadovski, O.; McCormick, T. M.; Babalhavaeji, A.; Tropepe, V.; Woolley, G. A., Photoswitching Azo Compounds in Vivo with Red Light. J. Am. Chem. Soc. 2013, 135, 9777-9784.

S4. Guo, Z.; Yu, J.; Zhang, Y.; Zhang, J.; Chen, Y.; Wu, Y.; Xie, L.; Li, J., Water-Stable In(III)-Based Metal-Organic Frameworks with Rod-Shaped Secondary Building Units: Single-Crystal to Single-Crystal Transformation and Selective Sorption of $\mathrm{C}_{2} \mathrm{H}_{2}$ over $\mathrm{CO}_{2}$ and $\mathrm{CH}_{4}$. Inorg. Chem. 2017, 56, 2188-2197.

S5. Wheate, N. J.; Anil Kumar, P. G.; Torres, A. M.; AldrichWright, J. R.; Price, W. S. J. xamination of Cucurbit[7]uril and Its Host-Guest Complexes by Diffusion Nuclear Magnetic Resonance. Phys. Chem. B 2008, 112, 2311-2314

S6. Grande, M. D. C.; García, M.; Marschoff, C. M. Density and Viscosity of Anhydrous Mixtures of Dimethylsulfoxide with Acetonitrile in the Range (298.15 to 318.15) K. J. Chem. Eng. Data 2009, 54, 652-658. 